\title{
A Segmented Output Stage H-Bridge IC with Tunable Gate DRIVER
}

by

Jingshu $\mathrm{Yu}$

A thesis submitted in conformity with the requirements for the degree of Master of Applied Science Graduate Department of Electrical and Computer Engineering University of Toronto

(C) Copyright 2014 by Jingshu Yu 


\begin{abstract}
A Segmented Output Stage H-Bridge IC with Tunable Gate Driver

Jingshu $\mathrm{Yu}$

Master of Applied Science

Graduate Department of Electrical and Computer Engineering

University of Toronto
\end{abstract}

2014

In this thesis, an integrated H-bridge is presented for continuously optimizing the power conversion efficiency over a wide range of output current. The proposed IC incorporates both segmented output transistors and segmented gate drivers. This fully segmentation approach allows to flexibly change the on-resistance of the output stage power transistors and the output resistance of the gate drivers. Dynamic adjustment of these parameters allows to investigate the best combination of output power transistor size and gate driver size for any given load current. The IC chip is fabricated using TSMC's $0.18 \mu \mathrm{m}$ BCD Gen-2 process technology. The presented design when operating as a buck converter with a load current between $0.02 \mathrm{~A}$ and $4 \mathrm{~A}$ achieves efficiency improvements of $32 \%$ and $8 \%$ at light and heavy load current, respectively. Furthermore, the dynamically adjustable gate driver output resistance allows for suppression of the peak CEMI by $5.5 \mathrm{dBm}$. 


\section{Acknowledgements}

I would like to express my sincere gratitude to several people without them this thesis might not be presented here.

Heartfelt gratitude to my supervisor, Professor Wai Tung Ng, who continues to provide guidance, inspiration and encouragement through the duration of my master program at University of Toronto. His support allows me to find and realize my potential in the area of integrated power electronics. His vision on the research area and close industry connection greatly help me to enhance the academic knowledge and develop technical skills. He is patient and sometimes strict as a mentor. He is intelligent and fun as a person. I feel fortunate to be accepted and work in his group.

I would also like to thank my fellow graduate students who have help build a perfect academic environment. I would like to name them here, who are Andrew Shorten, Jingxuan Chen, Weijia Zhang, Simon Jin, Rob Mckenzie and Sherrie Xie. Andrew's research on segmentation techniques provides a foundation for my thesis, and his generous help for layout portion and rich feedback during testing stage is sincerely appreciated. I am deeply grateful for the collaboration from Weijia, who offered thoughtful ideas and spent long days on the circuit design and testing for the research. An ISPSD conference paper will not be possible without her contribution. Loving thanks to Jingxuan and Jing Wang, whose friendship greatly helped my life transition to study abroad fast and smooth. Simon and Rob's previous research is very valuable for this new version of H-Bridge IC design.

I would like to thank NSERC Canada, and TSMC for the financial support and IC fabrication.

Last but not least, I would like to thank my dear parents Chunming Yu and Shui Zhou who played important roles along my twenty-four years journey. Your love, understanding

and support is invaluable. In particular, I would like to mention my boyfriend, Shengjie, for being an amazing part of my life ever since the day we first met. 


\section{Contents}

1 Introduction $\quad 1$

1.1 H-bridge Output Stage . . . . . . . . . . . . . . . . . 2

1.2 Power Losses in SMPS . . . . . . . . . . . . . . . . . 3

1.3 EMI in SMPS . . . . . . . . . . . . . . . . . . . . . . . . . . . . . . . . . .

1.4 Thesis Objectives and Organization . . . . . . . . . . . . 4

2 Background Research $\quad 6$

2.1 SMPS and Sychoronous DC-DC Buck Converter . . . . . . . . . . . . . 6

2.2 Different Power Losses in a Buck Converter . . . . . . . . . . . . . . 7

2.2.1 Conduction Loss . . . . . . . . . . . . . . . . . . . . . . . . . . 9

2.2 .2 Switching Loss . . . . . . . . . . . . . . . . . . . . 9

2.2 .3 Gate Driver Loss . . . . . . . . . . . . . . . . . . . . . 11

2.3 Impact of Packaging on IC Performance . . . . . . . . . . . . . . . . . 11

2.3.1 IC Package Type and Trends . . . . . . . . . . . . . . . . . 11

2.3.2 Package Parasitics . . . . . . . . . . . . . . . 12

2.3.3 Impact of Parasitics on Device Reliability . . . . . . . . . . . . . 14

2.4 EMI in SMPS . . . . . . . . . . . . . . . . . . . . 16

2.4.1 EMI Types and Effects on SMPS . . . . . . . . . . . 16

2.4.2 Sources of EMI in Buck Converter . . . . . . . . . . . . . . 17

2.5 Output Stage in SMPS . . . . . . . . . . . . . . . . . 18

2.5.1 Output Stage Configurations . . . . . . . . . . . . . 18

2.5.2 Integrated Output Stage Design . . . . . . . . . . . . . . . . 20

2.6 Gate Driver for the Power Transistor . . . . . . . . . . . . . . . . . . . . 22

2.7 Segmented Topologies . . . . . . . . . . . . . . . . . . 22

2.8 Summary . . . . . . . . . . . . . . . . . . 25

3 Segmentation Technique for the H-Bridge Design 26

3.1 Influence of $R_{\text {on }}$ of the Output Stage . . . . . . . . . . 26 
3.2 Influence of $R_{\text {out }}$ of the Gate Driver . . . . . . . . . . . . . 28

3.3 Optimized Operating Strategy of H-bridge . . . . . . . . . . . . . 31

3.4 System Topologies and Schematic Design . . . . . . . . . . . . . 33

3.4 .1 System Topologies . . . . . . . . . . . . . . . . 33

3.4 .2 Schematic Design . . . . . . . . . . . . . . . 35

3.5 Summary . . . . . . . . . . . . . . . . . . . . . 37

4 Implementation and Experimental Results $\quad 39$

4.1 IC Layout Design and Package Specifications . . . . . . . . . . . . . 39

4.2 On-resistance $R_{\text {on }}$ Measurements . . . . . . . . . . . . . . . 42

4.3 Experimental Setup . . . . . . . . . . . . . . . . . 45

4.4 Switching Speed and Ringing _. . . . . . . . . . . . . . 47

4.5 Power Conversion Efficiency Measurements . . . . . . . . . . . . . 48

4.6 Switching Node Ringing on EMI Issue . . . . . . . . . . . . . . 52

4.7 Summary . . . . . . . . . . . . . . . . . . 53

5 Conclusions $\quad 55$

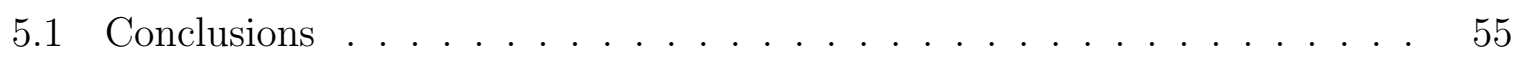

5.2 Future Work . . . . . . . . . . . . . . . . . . . . . . . 55

$\begin{array}{ll}\text { Bibliography } & 57\end{array}$ 


\section{List of Figures}

1.1 Block diagram for DC-DC power conversion [1]. . . . . . . . . . . . 1

1.2 Schematic of a synchronous buck converter $[1] \ldots \ldots \ldots \ldots$

1.3 An H-bridge consists of four $n$-type MOSFETs [2]. . . . . . . . . 3

2.1 A synchronous buck converter consists of two $n$-type MOSFETs [1]. . . 7

2.2 Voltage and current waveforms in buck-mode converter operation [1]. . . 8

2.3 Waveforms during the turn-on transition of a power transistor [1] . . . . 10

2.4 IC Package Development $[10] \ldots \ldots$

2.5 A simulation setup for parasitic inductances investigation. . . . . . . . . 14

2.6 Transient waveforms at the rising edge of $V_{s w} \ldots \ldots \ldots \ldots$

2.7 Influence of the parasitic inductances on the high-side transistor at the rising edge of $V_{s w} \ldots \ldots \ldots \ldots \ldots \ldots \ldots \ldots \ldots$

2.8 Influence of the parasitic inductances on the low-side transistor at the

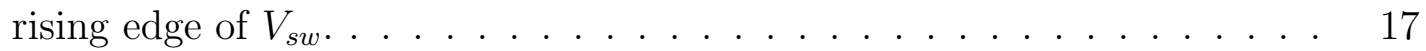

2.9 Influence of the parasitic inductances on the high-side transistor at the falling edge of $V_{s w} \ldots \ldots \ldots \ldots \ldots \ldots \ldots$

2.10 Influence of the parasitic inductances on the low-side transistor at the falling edge of $V_{s w} \ldots \ldots \ldots \ldots \ldots \ldots \ldots \ldots$

2.11 EMI sources in a typical DC-DC buck converter. . . . . . . . . . . . . 19

2.12 Common output stage configurations: (a) totem pole output stage, (b) push-pull output stage. . . . . . . . . . . . . . . 20

2.13 A general bootstrap circuit. . . . . . . . . . . . . . . . . . . . 21

2.14 Simplified circuit for (a) voltage driven (b) current driven (c) resonant

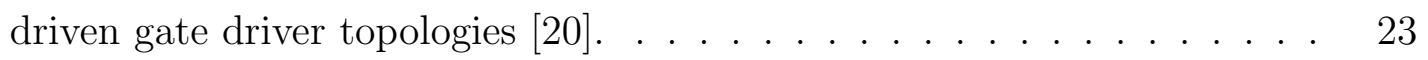

2.15 General schematic for a voltage driven gate driver [22]. . . . . . . . . . 24

2.16 A circuit configuration showing the segmented output stage [4] . . . . 24

2.17 A simplified diagram for the segmented gate driver [22] . . . . . . . . . 25 
3.1 A segmented power transistor which contains four identical $n$-EDMOS devices. . . . . . . . . . . . . . . . . 27

3.2 Efficiency versus load current for different enabled output stage segments. 29

3.3 Equivalent RLC circuit for the gate driver. . . . . . . . . . . . . 30

3.4 Segmented gate driver design. . . . . . . . . . . . . . . . 30

3.5 Efficiency versus load current for different gate driving strengths. . . . . 32

3.6 Timing diagram for the driving strategy. . . . . . . . . . . . . 33

3.7 Block Diagram of the Integrated Power H-bridge. . . . . . . . . . . . 34

3.8 Block diagram of one $n$-EDMOS with segmented gate driver arrangement. 34

3.9 Block diagram for driving the low-side $n$-EDMOS transistor. . . . . . 35

3.10 Data shift in the same direction as the clock signal. . . . . . . . . . . 36

3.11 Hold time problem. . . . . . . . . . . . . . . . . . . . . 36

3.12 Unit cell of the data shifting circuit. . . . . . . . . . . . . . . 37

3.13 General schematic of each gate driver segment. . . . . . . . . . . 38

4.1 A micrograph of the full layout for the H-bridge IC. . . . . . . . . . 40

4.2 Common centroid layout pattern for one power transistor. . . . . . . . 40

4.3 H-bridge IC mounted in the LCC84 package. . . . . . . . . . . . . . 41

4.4 Micrograph showing the wire bonding inside the LCC84 package. . . . . 41

4.5 Voltage potentials on Metal 4 layer as simulated using R3D. . . . . . . 42

4.6 Current density on the diffusion region as simulated using $R 3 D$. . . . 43

4.7 Post-layout resistance extraction report generated from $R 3 D$ simulation. 43

4.8 Simplified circuit diagram for $R_{o n}$ measurement. . . . . . . . . . . . 44

4.9 The buck converter circuit test bench. . . . . . . . . . . . . . 45

4.10 PCB implementation details. . . . . . . . . . . . . . 46

4.11 Overall experimental system setup. . . . . . . . . . . . . . 46

4.12 Switching node waveforms for different constant driving strength. . . . . 48

4.13 Rise time versus overshoot percentage. . . . . . . . . . . . . . 49

4.14 Switching node waveform for the proposed strategy. . . . . . . . . . 49

4.15 Power conversion efficiency versus load current for different gate driving

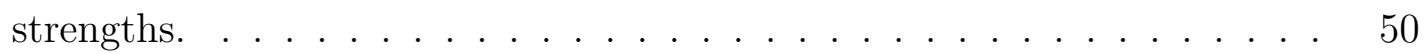

4.16 Power conversion efficiency versus load current for different output stage sizes. . . . . . . . . . . . . . . . . . 50

4.17 Efficiency versus load current for a half-bridge with the proposed operating strategy. . . . . . . . . . . . . . . . . . 51

4.18 CEMI spectrum for two typical driving strength at 1 A load current. . . 52 
4.19 Input Current waveforms for different driving strategies. . . . . . . . 53

4.20 CEMI spectrum for different driving strategies. . . . . . . . . . . . 54 


\section{List of Tables}

$1.1 \quad$ H-bridge Operating States $[2] \ldots \ldots \ldots$

2.1 Summary of IC Packages . . . . . . . . . . . . . . . . . . . 12

2.2 Abbreviations for Different IC Packages [12] . . . . . . . . . . . . 13

3.1 Characteristics of One $n$-EDMOS Segment . . . . . . . . . 27

3.2 Buck Converter Testing Parameters for Different $R_{o n} \ldots \ldots \ldots$

3.3 Buck Converter Testing Parameters for Different $R_{\text {out }} \ldots \ldots \ldots \ldots$

3.4 Proposed Operating Strategy _. . . . . . . . . . . . . . . . . 32

3.5 Gate Driver Segment Behaviors. . . . . . . . . . . . . . . . 38

4.1 Area Requirement for Primary Functional Blocks. . . . . . . . . . . . . 40

4.2 LCC84 Package Specifications. . . . . . . . . . . . . . . . . . . 41

4.3 Summary of $R_{o n}$ Measurements. . . . . . . . . . . . . . . . 44

4.4 Buck Converter Experimental Parameters. . . . . . . . . . . . . . . . 47 


\section{Glossary}

CEMI Conducted electromagnetic interference

EDMOS Extended drain MOSFET

EMI Electromagnetic interference

LCC Ceramic leadless chip carrier

PFM Pulse frequency modulation

PWM Pulse width modulation

SMPS Switched-mode power supplies

SOA Safe operation area 


\section{Chapter 1}

\section{Introduction}

A DC-DC converter, as shown in Fig. 1.1, is used to convert the one DC voltage to another DC voltage. Linear converter and switched-mode converter are two general methods to achieve the voltage conversion. The power conversion efficiency, defined as the ratio of the output power and the input power, is a good measure to evaluate the converter's performance [1]. Compared to the linear power supply, the switched-mode power supply (SMPS) is more efficient by using the passive components to store the energy.

Figure 2.1 shows a synchronous buck converter. By operating the power switches M1 and M2 alternately during a switching period, a voltage lower than the input voltage is obtained at the output node. The inductor $L$ is used to continuously provide the load current, and the capacitor $C_{\text {out }}$ is used to filter out the ripples in the output voltage.

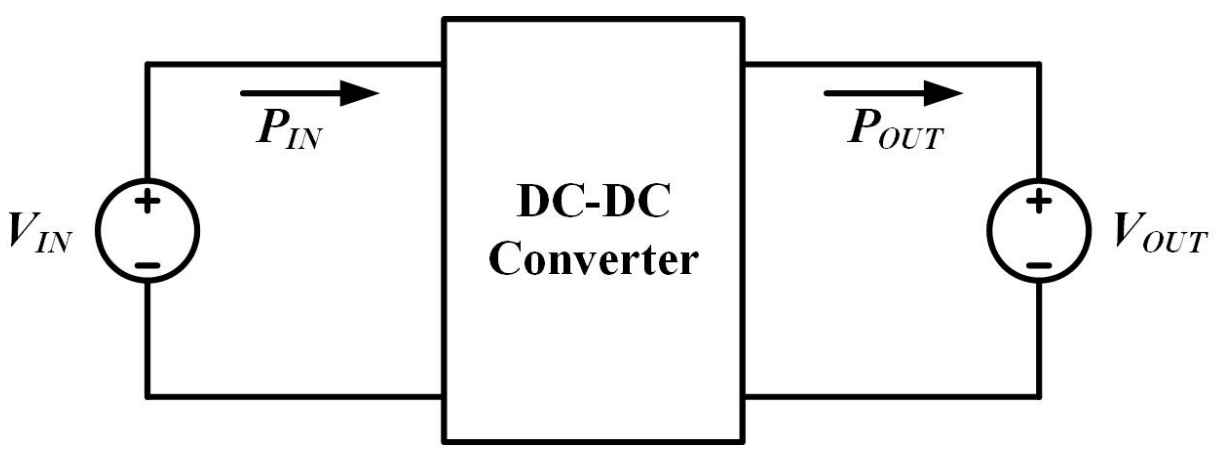

Figure 1.1: Block diagram for DC-DC power conversion [1]. 


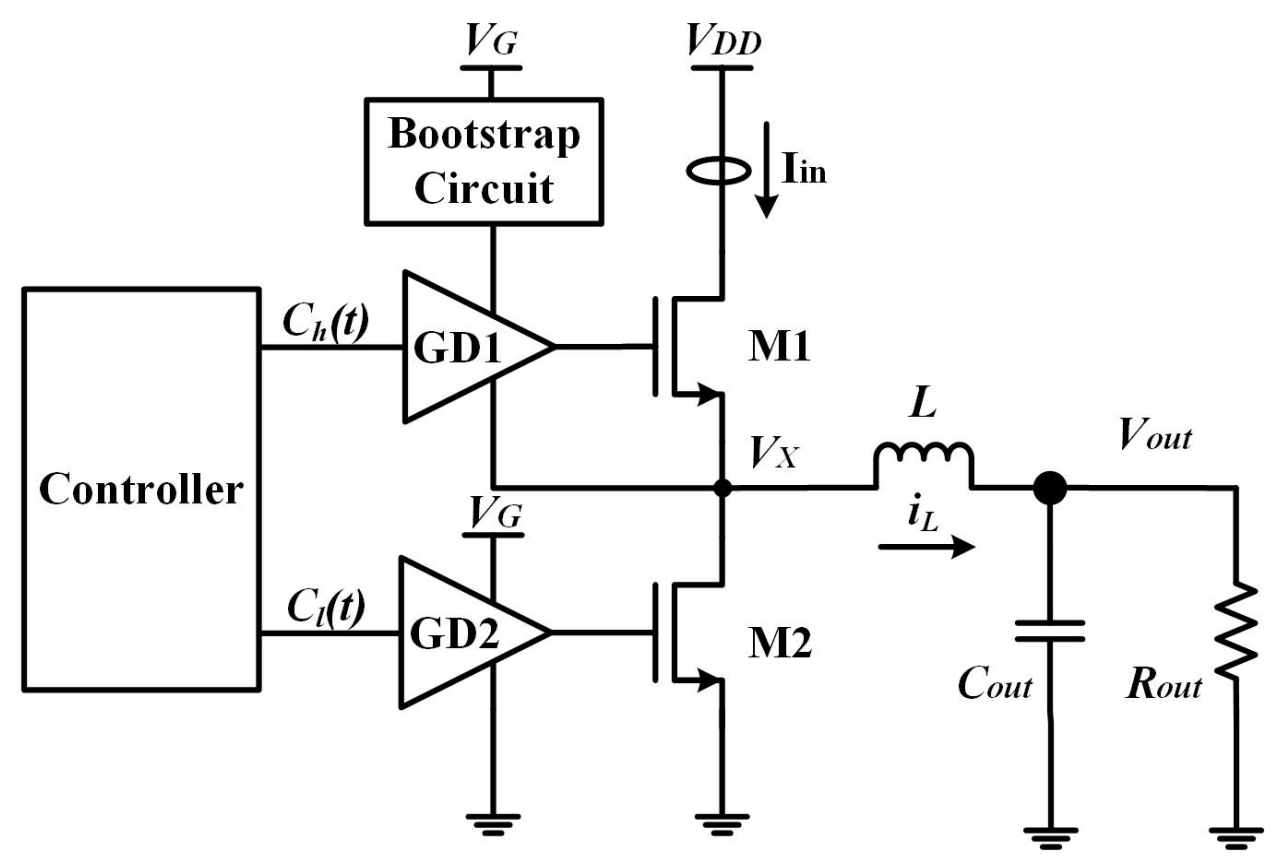

Figure 1.2: Schematic of a synchronous buck converter [1].

\section{$1.1 \quad$ H-bridge Output Stage}

The H-bridge topology is a highly versatile switching output stage for numerous modern applications such as Class-D audio amplifiers, motor drivers and DC-DC power conversions.

An H-bridge includes two identical output stages with four power switches, i.e. MOSFETs, IGBTs, etc.. Figure 1.3 shows a simple diagram of an H-bridge, with four n-type MOSFETs (M1, M2, M3, M4). If M1 and M4 are on, the other two power switches M2 and M4 are off, the load current flows from A to B in the direction. On the other hand, if M2 and M3 are on, M1 and M4 are off, the load is energized in the reverse direction. Therefore, H-bridge's operation is bidirectional. Table 1.1 summaries the four possible states that an H-bridge can operate. In State 3 and State 4, since of the power switches on the same side are on simultaneously, the power supply is shorted to ground and shootthrough happens. This condition is not desirable as since it can ruin the bridge and even the power supply [2].

A half-bridge is more simple, which only includes one side of the output stages with two power switches. Unlike an H-bridge discussed above, the direction of the current flowing through the load can only be in one direction.

As with any switched mode power supply, the overall power efficiency of an H-bridge is dependent on the design and the operation of the output stage and its gate driver. In 
Table 1.1: H-bridge Operating States [2].

\begin{tabular}{c|c|c|c|c|c}
\hline State & M1 & M2 & M3 & M4 & Description \\
\hline \hline 1 & on & off & off & on & $I_{L}$ direction: positive \\
\hline 2 & off & on & on & off & $I_{L}$ direction: reverse \\
\hline 3 & on & on & off & off & Shoot-through on the left side \\
\hline 4 & off & off & on & on & Shoot-through on the right side \\
\hline
\end{tabular}

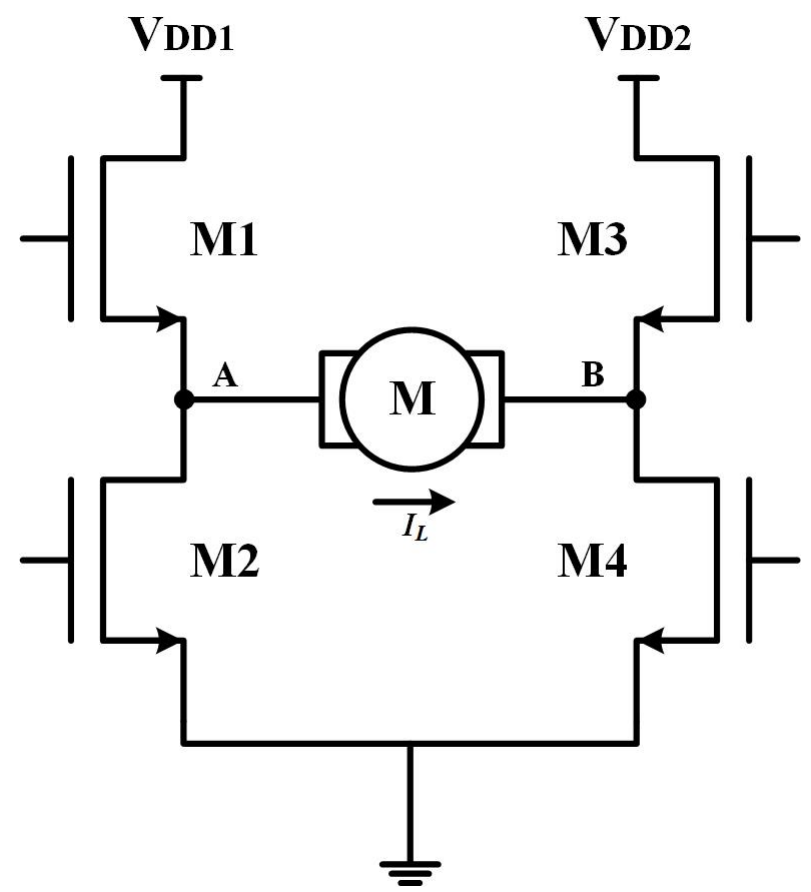

Figure 1.3: An H-bridge consists of four n-type MOSFETs [2].

addition, the nature of high frequency switching in SMPS could lead to the occurrence of electromagnetic interference (EMI). This may cause catastrophic issues in the operation of the SMPS and neighboring circuits. EMI mitigation with an efficient and economical method remains a challenge for IC designers.

\subsection{Power Losses in SMPS}

It is well known that conduction loss, switching loss and gate driver loss are three of the primary sources of power loss in SMPSs [1]. At light load conditions, the gate driver loss is dominant [3]. By decreasing the size of the output stage transistor, the charging and discharging of the gate capacitance for the power transistor can be reduced. This is 
subsequently leads to lower gate driver loss [4]. In addition, a small gate capacitance of the power transistor requires less driving effort from the gate driver, which reduces the unnecessary charing and discharging of the internal capacitive nodes. However, a small size power transistor implies a large on-resistance $\left(R_{o n}\right)$. When the SMPS is operating at high output current, the increased conduction loss degrades the power conversion efficiency [3]. In this case, a large gate driver is preferred since fast turn on and turn off times ( $t_{o n}$ and $t_{\text {off }}$, respectively) for the power transistor minimizes the switching loss and the shoot-through current. The observations above indicate that the overall power conversion efficiency of a traditional H-bridge can only be optimized for a particular operating condition.

\subsection{EMI in SMPS}

Periodically high frequency toggling of voltages and currents in SMPSs can cause EMI. This problem becomes a more serious concern in modern electronics when higher switching frequency (e.g. in 10's MHz range) is used to reduce component sizes and improve the transient performance. Therefore, minimizing the EMI to meet certain electromagnetic compatibility (EMC) standards is required before a SMPS product can be launched into the market. Widely accepted EMC regulations include Federal Communications Commission (FCC), British Standards (BS), Comite International Sepcial des Perturbations Radioelectrique (CISPR) and Verband Deutscher Elektrotechniket (VDE) [5].

The generation, propagation and common mitigation approaches of EMI are discussed in [6] and [5]. Two forms of EMI, including the conduted EMI (CEMI) and the radiated EMI, are generated by DC-DC converters. The CEMI noise only transmits along the current conducting power line, while the radiated EMI causes interference with the nearby electronic devices and systems. Two types of common CEMI mitigation approaches are categoried as follows, post-generation reduction and pre-generation elimination. The first method usually places a snubber circuit at the ringing node to filter the high-frequency noise. This approach is simple but not efficient due to the extra space, power dissipation and the cost for the passive components. As a result, the pre-generation elimination approach is preferred since it eliminates the excessive CEMI before it is generated [6].

\subsection{Thesis Objectives and Organization}

The objective of this thesis is to develop an integrated power output stage that can continuously optimize the power conversion efficiency of a typical DC-DC converter over 
a wide range of output current. The work also investigates the approach to suppress ringing oscillation at the switching node and CEMI without significant power efficiency degradation.

These two goals are demonstrated using an integrated H-bridge with segmented techniques utilized for both the power output transistors and the gate drivers. For any given load current, the best combination of the power output transistor size and the gate driver size can be adjusted on-the-fly. Furthermore, dynamic driving strategy similar to that of [7] is employed in order to reduce the CEMI for the H-bridge IC.

This thesis is organized as follows: Chapter 2 provides a background review on basic SMPS operations, EMI types and segmentation techniques; Chapter 3 presents the proposed H-bridge IC design and the dynamic operation strategy; Chapter 4 describes the IC implementation, the experimental setup, measurement approaches and experimental results for power efficiency optimization and CEMI reduction; Conclusions and future work are discussed in Chapter 5. 


\section{Chapter 2}

\section{Background Research}

This chapter reviews the power losses, EMI suppression and power conversion efficiency enhancement techniques.

\subsection{SMPS and Sychoronous DC-DC Buck Converter}

Compared to linear power supplies, SMPS can provide much higher power conversion efficiency [8]. Common types of SMPS include, buck, boost, buck-boost, Ćuk, and transformer-isolated such as fly-back converter [1]. In this thesis, the focus is to examine the switches' behavior during the transition periods and investigate the influence on the overall conversion performance in practical applications. The buck converter is selected for its simple operating mechanism.

A typical synchronous DC-DC buck converter is as shown in Fig. 2.1. The high-side (M1) and the low-side (M2) power transistors turn on and off alternately. Figure 2.2 illustrates the voltage and current waveforms for a basic operation condition. The conversion ratio $M(D)$ for the buck converter when operating in the continuous conduction mode $(C C M)$, can be expressed by

$$
M(D)=\frac{V_{\text {out }}}{V_{D D}}=D
$$

The ripples on the inductor current and the output voltage are given by (2.2) and (2.3), respectively. The expressions indicate that for a specific ripple tolerance, high switching frequency can reduce the passive components size, which implies small circuit board space for a converter. 


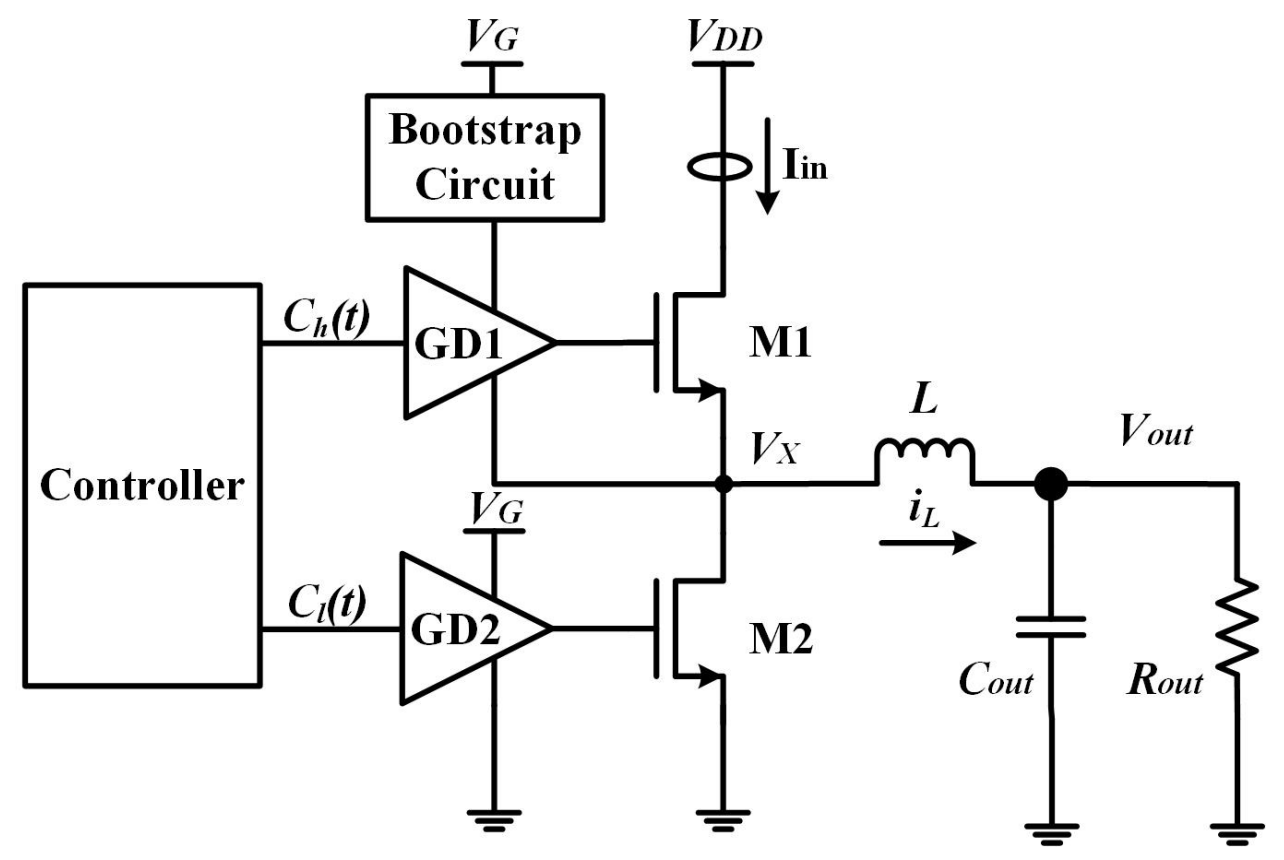

Figure 2.1: A synchronous buck converter consists of two $n$-type MOSFETs [1].

$$
\begin{gathered}
\Delta i_{L}=\frac{\left(V_{D D}-V_{\text {out }}\right) \cdot D}{2 L f_{s}} \\
\Delta v=\frac{\Delta i}{8 C f_{s}}
\end{gathered}
$$

\subsection{Different Power Losses in a Buck Converter}

The efficiency of a synchronous buck converter can be expressed as

$$
\eta=\frac{P_{\text {out }}}{P_{\text {in }}}
$$

For an ideal buck converter (as shown in Fig. 2.1), the theoretical power conversion efficiency can be $100 \%$. This means that the input power is totally transmitted to the load without any loss. However, in a real circuit, the efficiency is always degraded by power losses $P_{\text {loss }}$.

There are three main sources of $P_{\text {loss }}$ in a synchronous buck converter: the conduction loss $P_{\text {cond }}$, the switching loss $P_{s w}$ and the gate driver loss $P_{d r v}$ [9]. Other fixed power losses $P_{\text {fix }}$, such as the power consumed by the controller, are required to bias the circuit and independent of the switching frequency. $P_{f i x}$ is often neglected at heavy load or high 


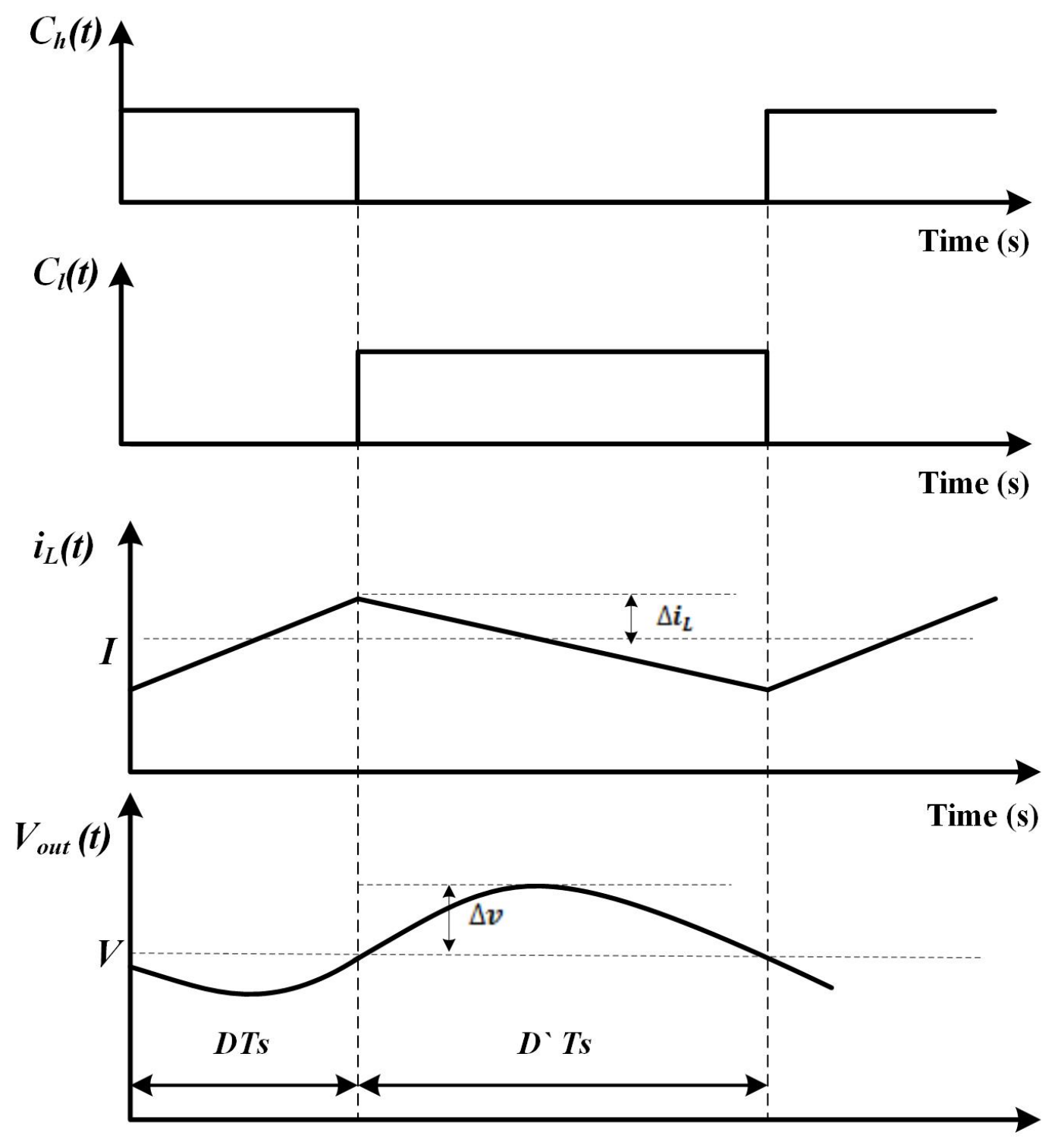

Time (s)

Figure 2.2: Voltage and current waveforms in buck-mode converter operation [1]. 
switching frequency situation due to its small value when compared to the other three.

\subsubsection{Conduction Loss}

According to Section 2.1, the high-side and low-side transistors carry the conducting inductor current alternately in a switching period. In a buck converter, when a transistor is on, it can be viewed as a resistor with effective on-resistance of $R_{o n}$. The conduction loss due to this on-resistance can be calculated as

$$
P_{\text {cond }}=I_{L}^{2} \cdot R_{\text {on_hs }} \cdot D+I_{L}^{2} \cdot R_{\text {on_ls }} \cdot(1-D)
$$

Assuming that the high-side and low-side transistors are identical, (2.5) can be simplified as follows

$$
\begin{gathered}
P_{\text {cond }}=I_{L}^{2} \cdot R_{o n} \\
R_{o n}=R_{s h} \cdot \frac{L}{W}
\end{gathered}
$$

where $R_{s h}$ is the sheet resistance of the channel. $L$ is the gate length and $W$ is the width of the transistor. Since $R_{s h}$ is determined by the fabrication technology and the minimum gate length is usually used during IC design, these two parameters can be considered as fixed features [9]. Therefore, the transistor width $W$ is the main variable for the on-resistance $R_{o n}$. Equations (2.6) and (2.7) show that the conduction loss $P_{\text {cond }}$ and the on-resistance $R_{o n}$ have an inversely proportional relationship to the transistor width $W$. Therefore, a large $W$ for the power transistor implies a smaller conduction power loss.

\subsubsection{Switching Loss}

Switching loss is caused by the loss in the power transistor during the transition period. The transistor cannot be switched between on and off states instantaneously. It requires a certain time to complete the switching action. As shown in Fig. 2.3, during the turnon transition, both current and voltage values are non-zero. The overlap results in a transistor switching loss $P_{s w}$ [1]. During the turn-off transition, the power loss is similar to the waveforms as shown in Fig. 2.3. Therefore, the total switching loss is given by

$$
P_{s w}=f_{s w} \cdot\left(\int _ { t u r n - o n } \left(v_{d s}(t) \cdot i_{d s}(t)+\int_{t u r n-o f f}\left(v_{d s}(t) \cdot i_{d s}(t)\right)\right.\right.
$$

Assuming that the voltage and current waveforms are linear, the switching loss can 


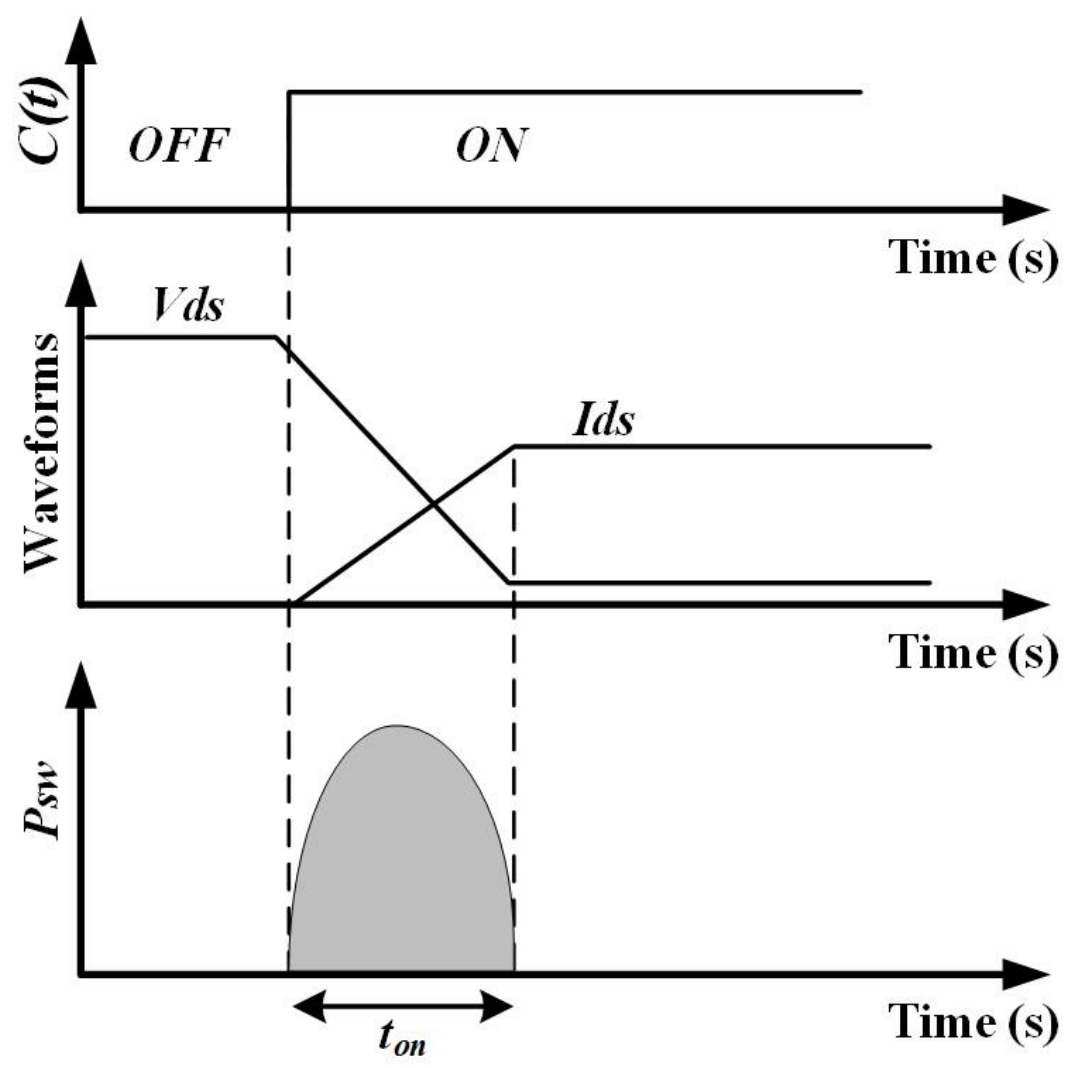

Figure 2.3: Waveforms during the turn-on transition of a power transistor [1]. 
be calculated by [1]

$$
P_{s w}=f_{s w} \cdot V_{d s} \cdot I_{L} \cdot\left(\frac{t_{o n}+t_{o f f}}{2}\right)
$$

\subsubsection{Gate Driver Loss}

Gate driving loss $P_{d r v}$ due to the charging and discharging of the capacitance internal to the gate driver is another important power loss.

$$
\begin{gathered}
P_{d r v}=f_{s w} \cdot V_{G}{ }^{2} \cdot\left(C_{g s_{-} h s}+C_{g s_{-} l s}\right) \\
C_{g s}=C_{g s, s p} \cdot W \cdot L
\end{gathered}
$$

where $C_{g s \_h s}$ and $C_{g s \_l s}$ refer to the input gate capacitance of high-side and low-side transistors, respectively. Since $C_{g s, s p}$ is another technology specific parameter which cannot be altered by the IC designers [9], $C_{g s}$ is proportional to the transistor width $W$. Therefore, a power transistor of minimum usable size is preferred so that the gate driver loss can be reduced.

Recapping the relationship between $R_{o n}$ and $W$, a trade-off exists between $R_{o n}$ and $C_{g s}$. For a specific fabrication, a figure of merit (FOM) defined as the product of onresistance $R_{o n}$ and gate charge $Q_{g}$ can be used to find an optimized transistor width, so that the total power loss can be minimized.

\subsection{Impact of Packaging on IC Performance}

The bare unpackaged silicon die is very vulnerable to the environment. High temperature and humidity from the outside environment can lead to oxidization and malfunction of the chip. Therefore, packaging is an essential part of the IC design. The IC package not only acts like a protector, but also as a carrier for the chip. A good packaging should have characteristics of small size, low cost, mechanical reliability, and high electrical performance [10].

\subsubsection{IC Package Type and Trends}

The IC package type can be classified in different ways, e.g. by material, by mounting method, by lead type, etc.. Table 2.1 is a summary of different IC package types [10], [11].

Driven by the increasing demand of high number of I/O pins, high power density, high reliability and performance requirements, the IC packaging technology has enjoyed 
Table 2.1: Summary of IC Packages

\begin{tabular}{llll}
\hline & Description & Features & Examples \\
\hline \hline Materials & & & \\
\hline Plastic & plastic package & cheap & PLCC \\
Ceramic & ceramic package & good heat dissipation & PGA LCC \\
\hline Mounting Method & & \\
\hline Through Hole & drill hole needed & Cheap;Easy to assembly & DIP ZIP \\
Surface Mount & one side attched only & Space saving; & SOJ QFN BGA \\
\hline Lead Type & & & \\
\hline Leaded & Gull-wing; J-Lead & Easy to solder & QFP SOJ \\
Leadless & Lead-free & Higher I/O counts & LCC QFN \\
\hline Pin Arrangement & & \\
\hline $\begin{array}{l}\text { Small Outline } \\
\text { Quad Flat Pack }\end{array}$ & Dual line pin & widely used & SOIC TSOP \\
Grid Array & Flip-chip Assembly & Compact size & QFN QFP LCC \\
\hline
\end{tabular}

significant advancement. The history of the IC packaging can be traced back to Dual In-Line Package (DIP) in 1970's. Through-hole mounting is a classical approach, which is inexpensive and easy to implement. With higher level of integration density, this kind of packaging is gradually replaced by the surface mount technology (SMT). SMT has the benefits of lower parasitics, higher reliability and space saving for complex PCB design. Figure 2.4 shows that miniaturization and higher pin-count is the trend for IC packaging development [10]. Table 2.2 provides the definition for package type abbreviations mentioned in this section.

\subsubsection{Package Parasitics}

For integrated chip testing on printed circuit board (PCB), there are two levels of connection between the silicon die and board: chip to package and package to PCB. Parasitic capacitance, inductance and resistance are introduced to the system from wire-bonding and PCB traces. The influence from these parasitics is becoming a serious issue for integrated output stage with power transistors of small $R_{o n}$ [13]. In addition, these parasitic elements can affect the overall electrical performance of the chip, by unwanted oscillations at various circuit nodes [14]. Voltage and current ringings during the switching transition may result in power device operating out of safe operation area (SOA), and 


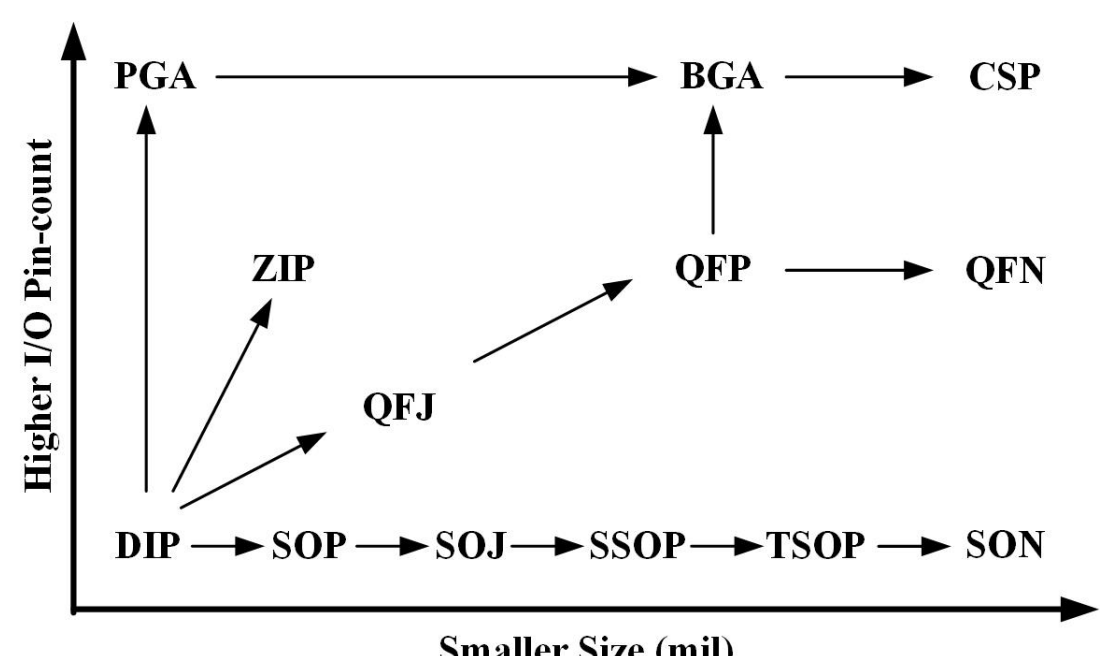

Figure 2.4: IC Package Development [10]

Table 2.2: Abbreviations for Different IC Packages [12].

\begin{tabular}{c|c}
\hline Abbreviation & Package Type Description \\
\hline \hline BGA & Ball Grid Array \\
\hline LCC & Ceramic Leadless Chip Carrier \\
\hline CSP & Chip Scale Package \\
\hline DIP & Dual-in-Line Package \\
\hline PGA & Pin Grid Array \\
\hline PLCC & Plastic Leaded Chip Carrier \\
\hline QFJ & Quad Flat J-Leaded Package \\
\hline QFN & Quad Flat No-Leaded \\
\hline QFP & Quad Flat Pack \\
\hline SOIC & Small Outline IC Package \\
\hline SOJ & Small Outline J-Leaded Package \\
\hline SON & Small Outline Non-Leaded Package \\
\hline SOP & Small Outline Package \\
\hline SSOP & Shrink Plastic Dual-in-Line Package \\
\hline TSOP & Thin Small Outline Package \\
\hline ZIP & Zigzag-in-Line Package \\
\hline
\end{tabular}




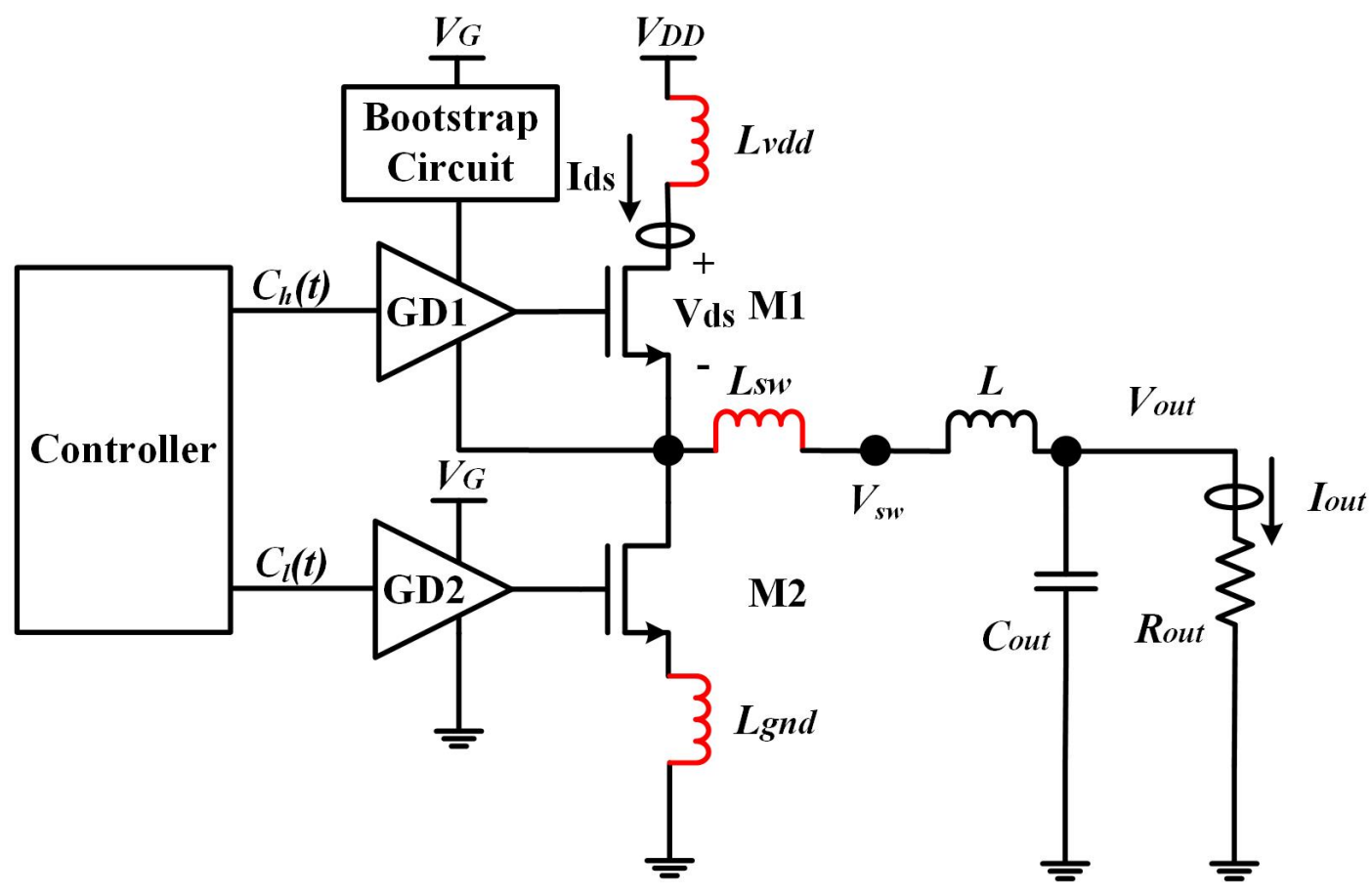

Figure 2.5: A simulation setup for parasitic inductances investigation.

subsequently reduce the lifetime of the device. In addition, the oscillations in the current waveform produce conducted EMI, and degrades the EMC of the products. The impact of ringing oscillation will be discussed in Section 2.4

\subsubsection{Impact of Parasitics on Device Reliability}

In order to acquire a better understanding on the impact of the parasitic components, the parasitic inductances from the DIP package are estimated and added to the model of a DC-DC buck converter in simulation. Figure 2.5 shows the simulation setup: the parasitic inductances $L_{v d d}, L_{g n d}$ and $L_{s w}$ are estimated based on an output stage IC mounted in a typical DIP package. Assuming that the output node of the gate driver is directly connected to the gate terminal of the power transistor, parasitic inductances from this path is neglected. The output stage consists of two identical $n$-MOS transistors, M1 and M2. The drain-source voltage $V_{d s}$, the drain current $I_{d s}$, and the switching node (SN) voltage $V_{s w}$ are recorded during the switching period of each device in the simulation. The impacts of three variables were individually investigated by increasing the value of Lvdd Lgnd and Lsw.

Figure 2.6 plots the transient waveforms for the high-side transistor when it turns on. The operating area, which focuses on the locus of the current and voltage transition 

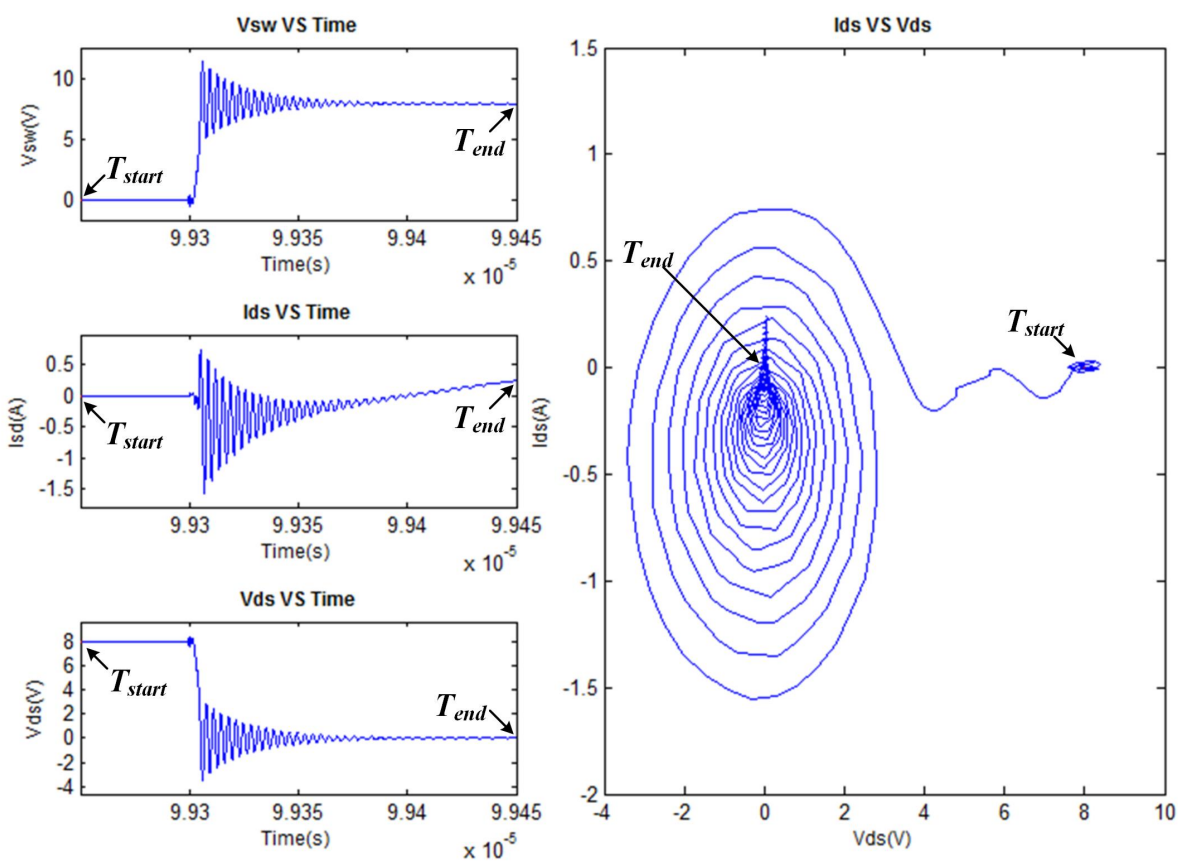

Figure 2.6: Transient waveforms at the rising edge of $V_{s w}$.

behavior of the power transistor is plotted on the right in the same graph. Ringings are observed at the beginning of the transition. In order to investigate the influence of the parasitic inductance, the value of each inductance is varied and re-simulated. The operating area for the high-side transistor is summarized in Fig. 2.7. With a doubling in the size of $L v d d$, more $V d s$ ringing is produced, but $I d s$ oscillation is reduced at the same time. With a doubling in the size of Lgnd, more $V d s$ ringing is produced at the beginning of the transition period, but becomes less at the end of the transition. The value of $L s w$ does not cause a big difference since it can be ignored when compared to the large output inductor in the buck converter.

Similar plots for the low-side transistor at the rising edge of $V_{s w}$ are as shown in Fig. 2.8. Figures 2.9 and 2.10 show the transition behavior for the high-side transistor and the low-side transistor at the falling edge of $V_{s w}$, respectively. Simulation results show that Lvdd leads to ringing oscillation at the switching node; Lgnd leads to ringing oscillation during pull up; Lsw, however, has little effects on the transistor operation. Excessive ringing oscillation at the switching node degrades the performance of the circuit and possibly damage the transistor if the $I-V$ curve goes outside the safe operation area (SOA) for some time during switching. Current oscillation, on the other hand, can results in EMI issues. Therefore, an appropriate package is a vital factor that guarantees the performance of the chip. 

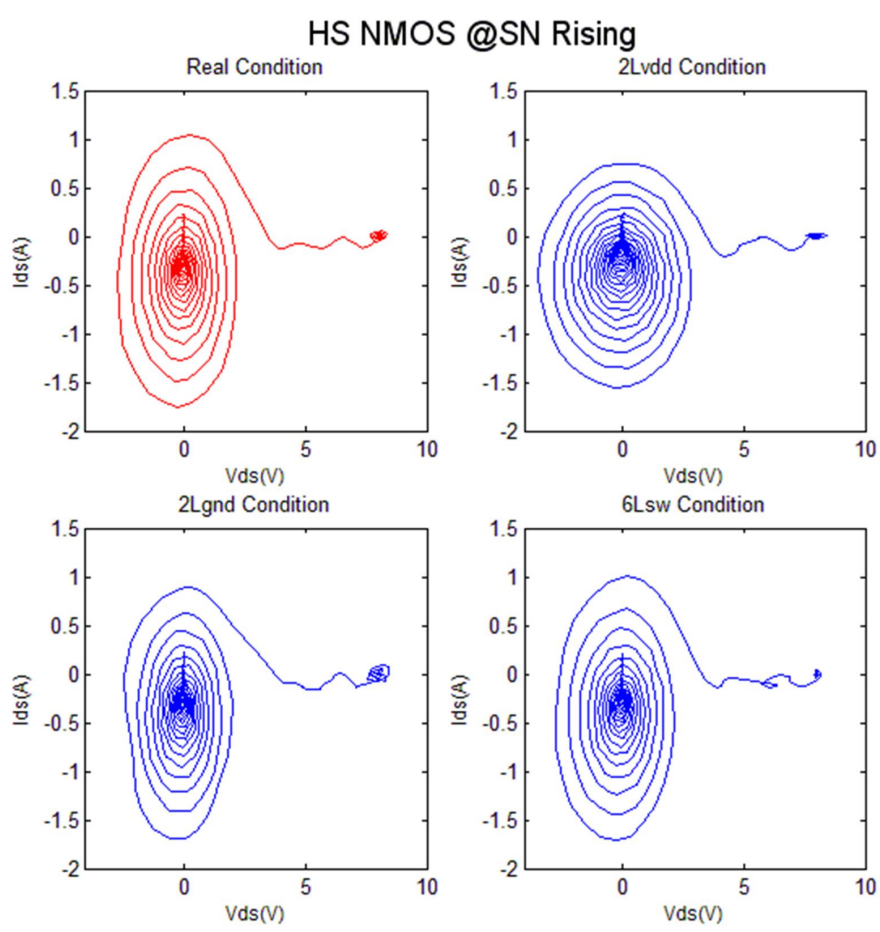

Figure 2.7: Influence of the parasitic inductances on the high-side transistor at the rising edge of $V_{s w}$.

\section{$2.4 \quad$ EMI in SMPS}

SMPS integrated circuits generate significant amount of electromagnetic noise due to its high frequency switching. Negative impacts including performance degradation, or even system malfunction become a big concern for the IC designers [15]. Therefore, understanding the EMI generating mechanism in SMPS and the corresponding mitigation techniques can help IC designers to reduce the effect of the disturbance.

\subsubsection{EMI Types and Effects on SMPS}

Generally, electromagnetic interference (EMI) in SMPS can be classified into two types: conducted EMI and radiated EMI [16].

The conducted EMI is produced and propagated along the current conducting path. In a SMPS system, voltage at the switching node and input current exhibit the fast switching characteristics, which bring about unwanted harmonics and high frequency noise. Conducted EMI noise generated from high frequency switching is then coupled to the other circuits connected on the same supply line. Radiated EMI is transmitted into all directions in the form of electromagnetic waves. It disturbs the nearby devices which 

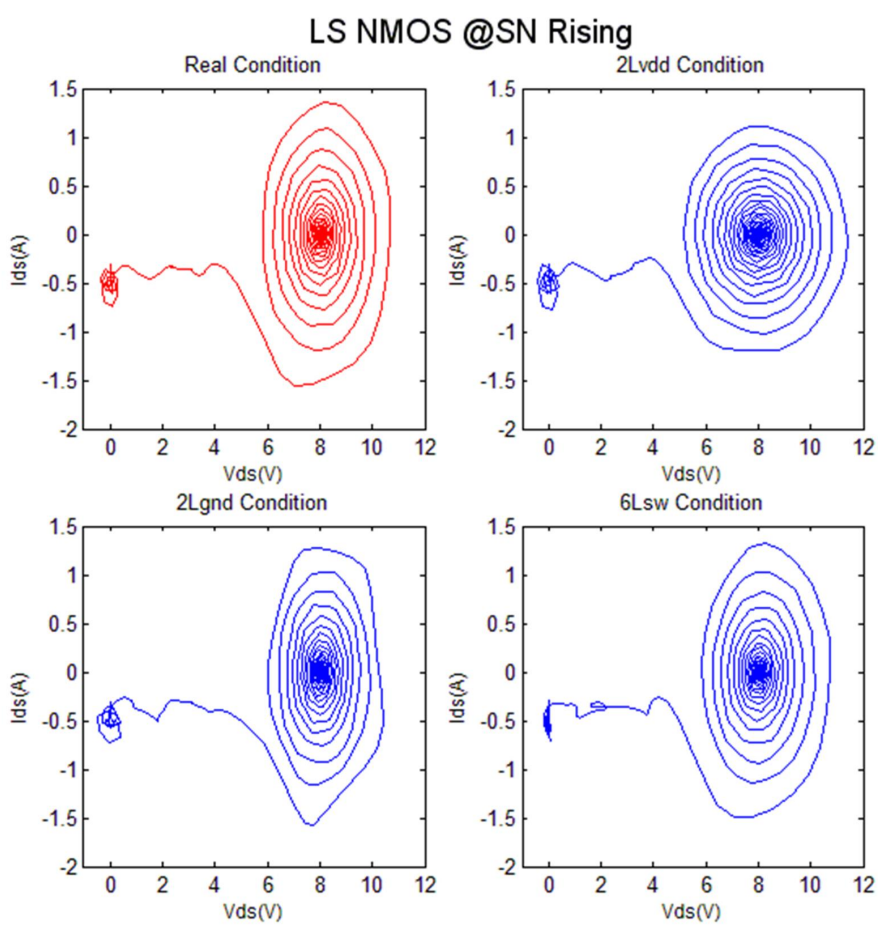

Figure 2.8: Influence of the parasitic inductances on the low-side transistor at the rising edge of $V_{s w}$.

are exposed to this electromagnetic field. However, an effective antenna is not easy to be defined or recognized. Therefore, a bond wire inside the IC package or a PCB trace can be a source of radiated EMI [17].

\subsubsection{Sources of EMI in Buck Converter}

EMI in DC-DC buck converters mainly results from the fast switching of voltages and currents. As shown in Fig. 2.11, the pulse-width-modulation (PWM) signal at the gate node of the power transistor, the voltage ringing at the switching node and the input current oscillation on the input power line are square waveforms with high switching frequency. These three are the main sources of EMI in the buck converter circuit.

PWM is the most common approach to drive the output stage in the buck converter. However, due to the input capacitance $C g s$ of the power device, the parasitic inductance from the PCB layout and the output resistance $R_{\text {out }}$ of the gate driver, the RLC circuit generates the voltage ringing at the gate node of the power transistor and subsequently at the switching node in the buck converter. As shown in Fig. 2.11, if the input current is periodically flowing into the buck converter with a duty ratio of $D$ and output current of $I$, the Fourier transformation of the input current shows EMI at frequencies well the 

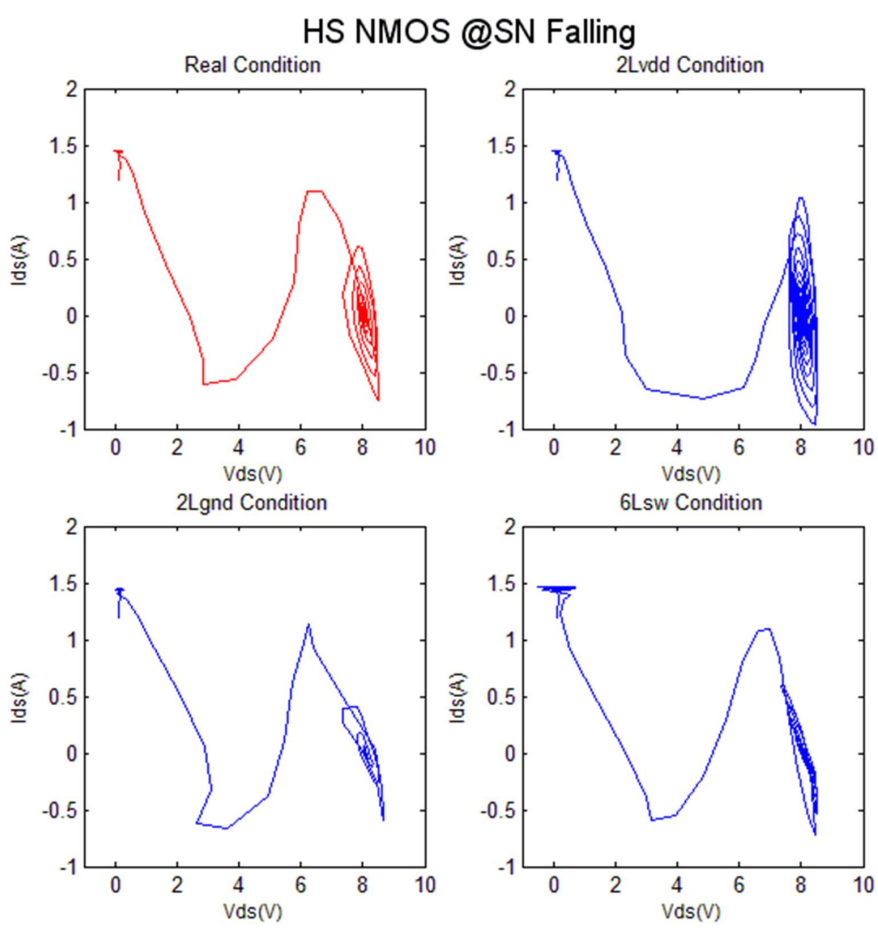

Figure 2.9: Influence of the parasitic inductances on the high-side transistor at the falling edge of $V_{s w}$.

PWM frequency and its harmonics [18].

$$
f(t)=D I+\sum_{i=1}^{\infty}\left(\frac{2 I}{k \pi} \sin (D k \pi) \cos (k \omega t)\right)
$$

\subsection{Output Stage in SMPS}

An output stage normally contains two power switches [19]. Two common output stage configurations and the output stage IC design are described in this section.

\subsubsection{Output Stage Configurations}

Fig. 2.12 illustrates the two most widely used output stage configurations: totem-pole output stage and push-pull complementary output stage. The totem-pole output stage consists of two stacked $n$-type MOSFET ( $n$-MOS), while the push-pull complementary output stage consists of one $p$-type MOSFET ( $p$-MOS) and one $n$-MO [19].

For both configurations, the low-side transistor is $n$-MOS with source and body connected to the ground, drain connected to the switching $V_{X}$ node. The choice between 

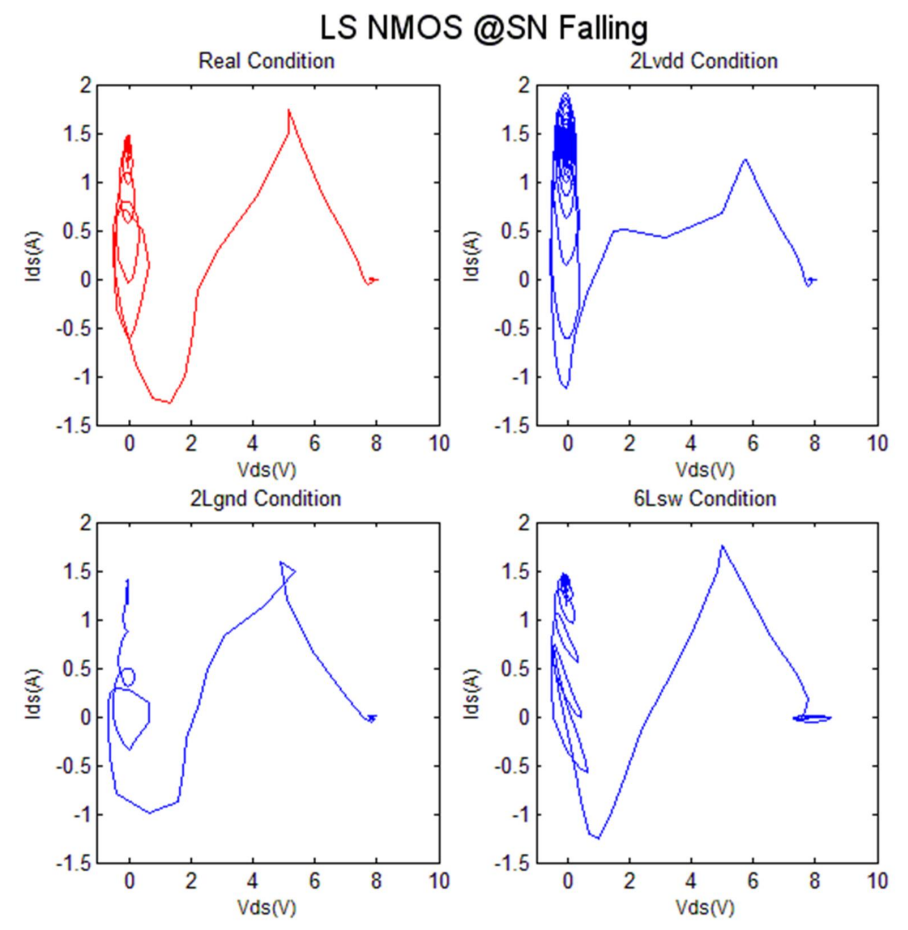

Figure 2.10: Influence of the parasitic inductances on the low-side transistor at the falling edge of $V_{s w}$.

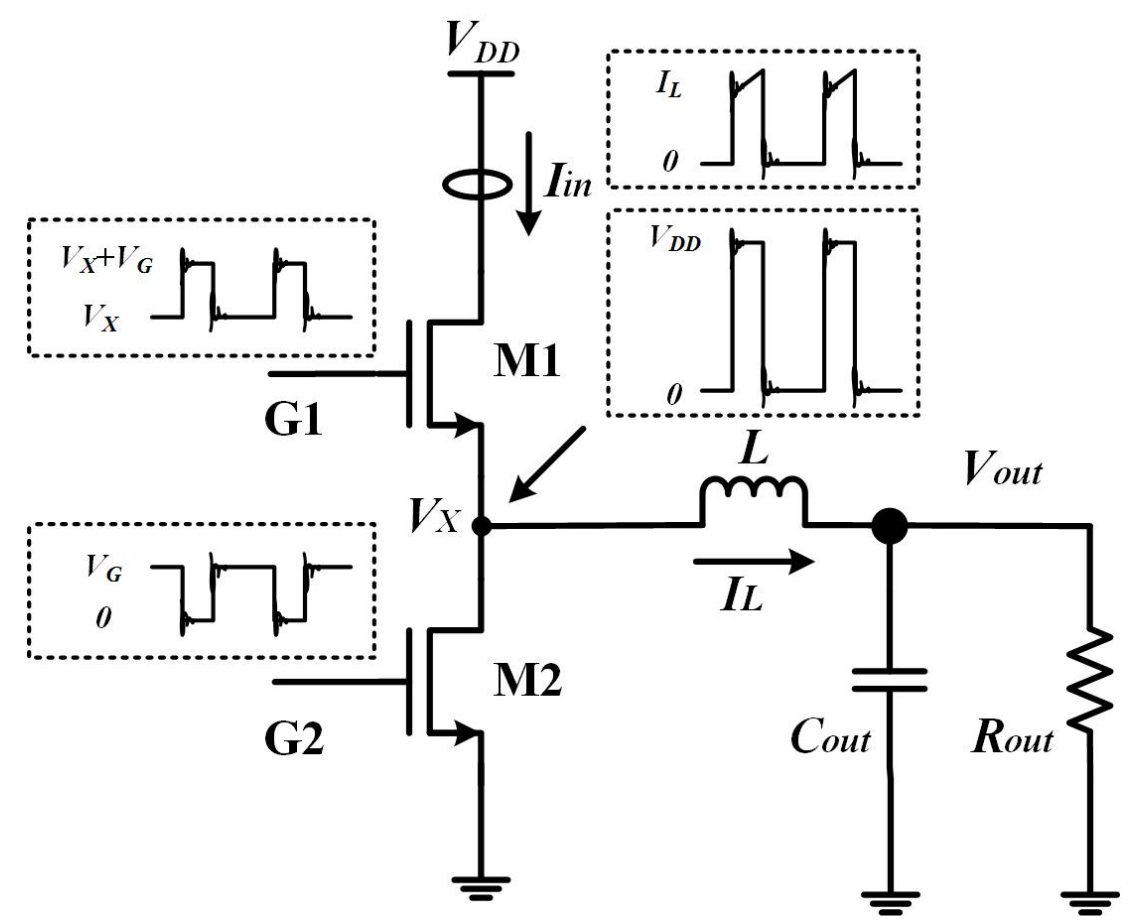

Figure 2.11: EMI sources in a typical DC-DC buck converter. 


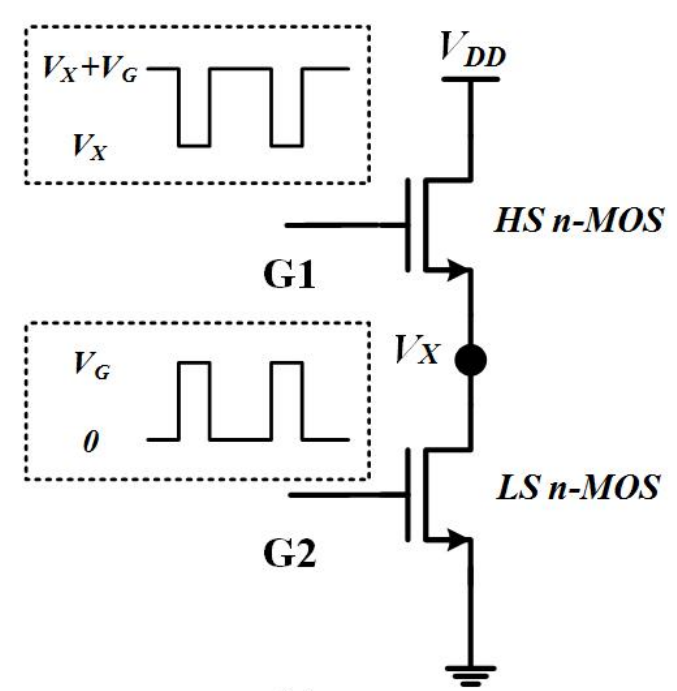

(a)

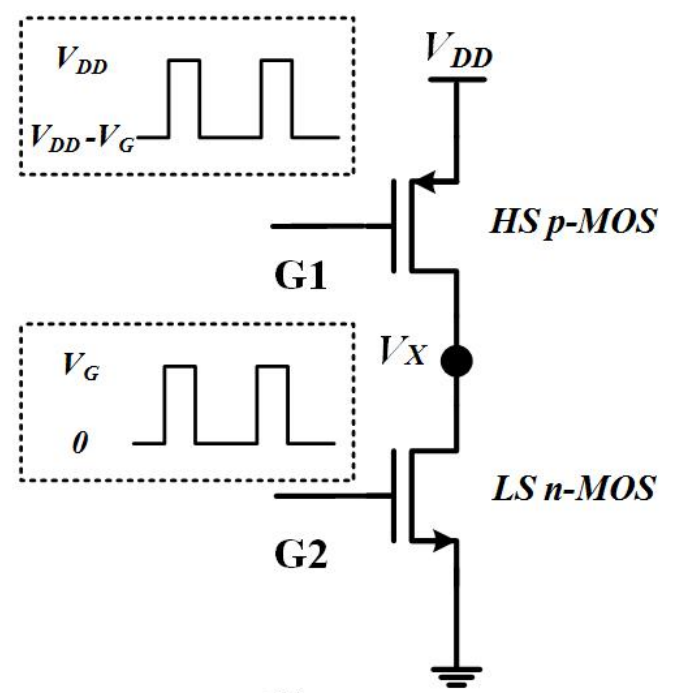

(b)

Figure 2.12: Common output stage configurations: (a) totem pole output stage, (b) push-pull output stage.

these two output stage configurations is mainly dependent on the target output power. For a given $R_{o n}$ for the power transistor, the $p$-MOS power transistor occupies a larger area than an $n$-MOS due to its lower hole carrier mobility. Considering the increasing requirement of high power level and small device area, the totem-pole configuration with two $n$-MOS power transistors is more popular in integrated SMPS designs. However, the totem-pole output configuration has an intrinsic drawback: the floating source of the high-side transistor. As shown in Fig. 2.12, since the source of the high-side $n$-MOS is tied to the output node $V_{X}$, the source voltage is floating and toggling between the ground and $V_{D D}$ at the switching frequency. In order to turn on the high-side $n$-MOS, the high-side gate driver needs to provide a driving voltage higher than the supply voltage. Therefore, a bootstrap circuit (as shown in Fig. 2.13) is required to bias the high-side gate driver circuit.

\subsubsection{Integrated Output Stage Design}

There are many parameters need to be considered for an output stage IC design. The key factors include the output stage configuration, the on-resistance $R_{o n}$, the input capacitance $C_{g s}$, the switching speed $f_{s}$, the device area, etc..

Normally, for low output power applications, the push-pull complementary output stage is more appropriate due to its simplicity. The low hole carrier mobility issue does not matter much when duty ratio $D$ is low since the $p$-MOS on the high-side is 


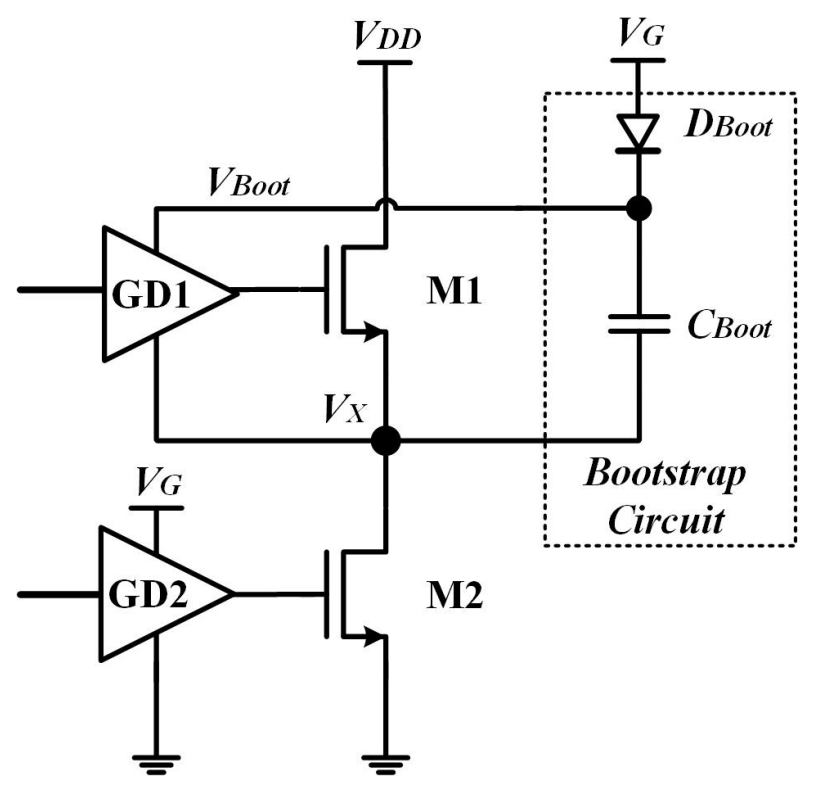

Figure 2.13: A general bootstrap circuit.

conducting current for a very short time. On the contrary, for high voltage and heavy load applications, the totem-pole configuration is preferred due to its smaller area for a given $R_{\text {on }}$ requirement [19].

On-resistance $R_{o n}$, input capacitance $C_{g s}$, device area and other related parameters mentioned above must be taken into account when choosing the size of the power transistors. Since the sheet resistance $R_{s h}$ is determined by the technology process, according to (2.7), the on-resistance of an $n$-MOS is inversely proportional to the width $W$ of the transistor provided minimum gate length $L$ is used. However, (2.14) indicates a large transistor width would lead to large $C_{g s}$, which means a long charging or discharging time for a specific driving strength. So, a small on-resistance $R_{o n}$ and a low input capacitance $C_{g s}$ cannot be achieved at the same time. Power loss calculations based on the analytical model as discussed in Section 2.2, and circuit simulation can help to find an optimized transistor width to minimize the power loss for a specific load current.

$$
\begin{aligned}
& R_{d s, o n}=\left(\frac{\rho}{t}\right)\left(\frac{L}{W}\right)=R_{s} \frac{L}{W} \\
& C_{g s}=W L\left(\frac{\varepsilon_{o x}}{t_{o x}}\right)=W L C_{o x}
\end{aligned}
$$

The target switching speed $f_{s}$ is another important design specification. It is dependent on the input capacitance $C_{g s}$ and the gate driver driving ability. This part will be further discussed in Section 2.6. 


\subsection{Gate Driver for the Power Transistor}

Gate driver design is a critical part for integrated power transistors. In order to turn the power transistors on and off, the gate driver must supply enough current to charge and discharge the gate capacitance $C_{g s}$. As shown in Fig. 2.14, the gate driver topology can be categorized into three groups: voltage driven gate driver, current driven gate driver, and resonance driven gate driver [20].

Among these three types of gate driver topologies, the current driven gate driver is seldom used due to a high efficiency current source requirement [20]. The resonant driven topology is popular for high frequency applications. It has the advantage to recover the power loss when charging and discharging the gate capacitance [21]. However, the extra passive components is not desirable for space and cost saving purpose in the integrated output stage design. Therefore, the voltage driven gate driver is the most widely used topology. In the simplified equivalent circuit as shown in Fig. 2.14 (a), the input capacitance of the power transistor $C_{g s}$ is charged by a DC voltage source through a effective resistance $R_{\text {out }}$.

Figure 2.15 shows the schematic of voltage driven gate driver topology, which contains an inverter chain. The gate driver loss due to the charging and discharging of capacitances internal to the gate driver is given by [22]

$$
P_{g d}=f_{s} \cdot V_{G} \cdot\left(\frac{f^{N}-1}{f-1}\right) \cdot\left(C_{\text {in }}+C_{\text {out }}\right)+P_{\text {static }}
$$

Equation (2.15) indicates that a long inverter chain consumes more power when compared to a short one. The chain length, namely the stage count $N$, is determined by the input capacitance $C_{g s}$ of the power transistor seen at the output node of the gate driver.

\subsection{Segmented Topologies}

According to Section 2.5.2, the size of the output stage is determined by the desired output power level [23]. The power loss, dominated by the conduction loss and the gate driving loss can be minimized for a specific operating condition. However, in order to continuously optimize the power conversion efficiency of a DC-DC buck converter over a wide range of load current, an output stage with transistors of fixed size is not desired. Segmentation is one approach to achieve the size changing capability.

The idea of segmented topology was first presented by [4]. This topology was employed for the output stage which allows to digitally adjust the width of the output transistor 


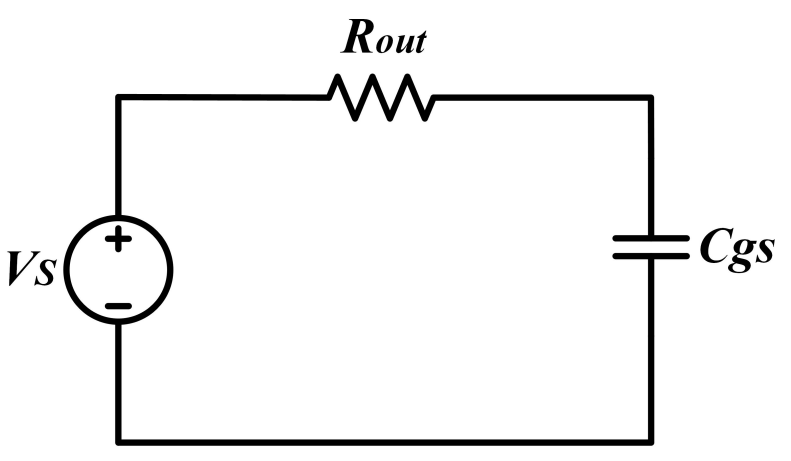

(a)

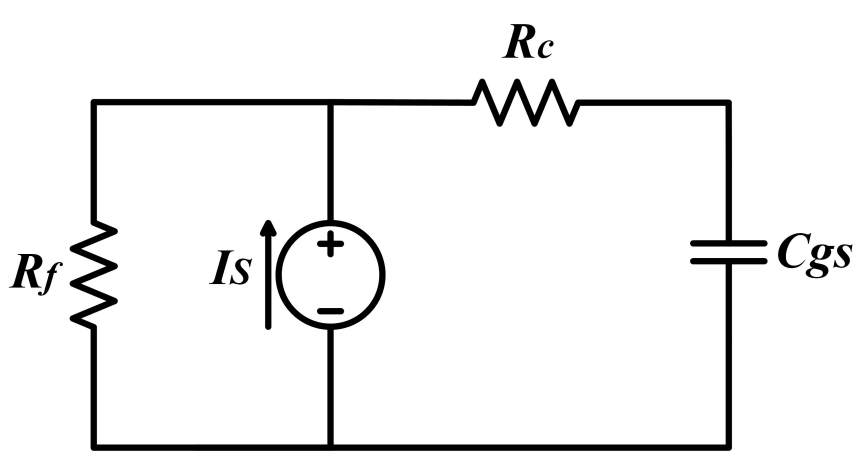

(b)

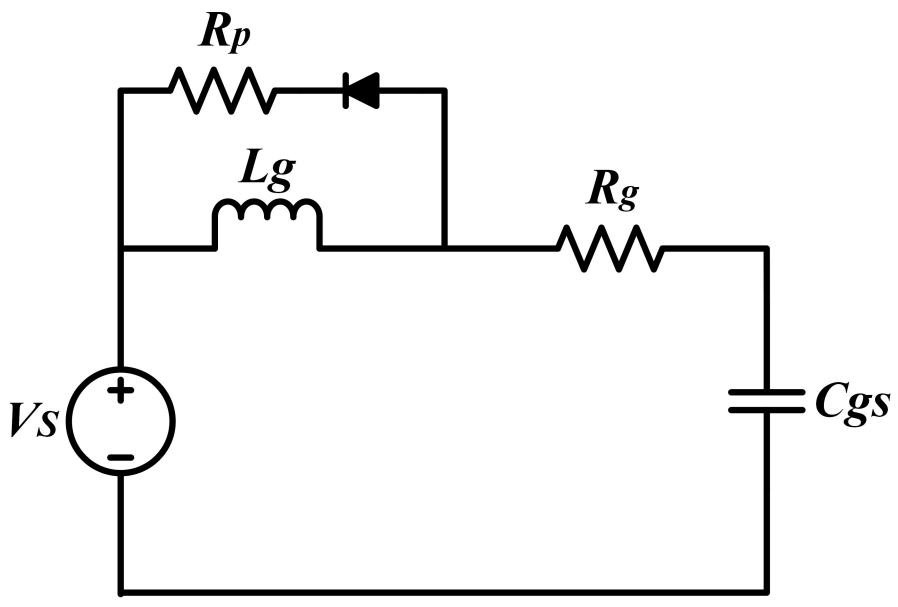

(c)

Figure 2.14: Simplified circuit for (a) voltage driven (b) current driven (c) resonant driven gate driver topologies [20]. 


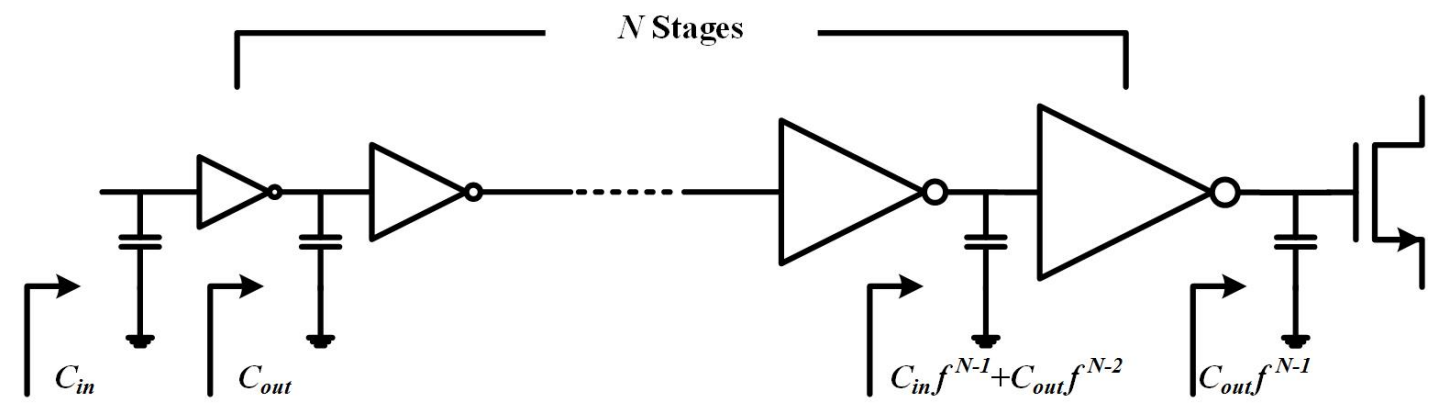

Figure 2.15: General schematic for a voltage driven gate driver [22].

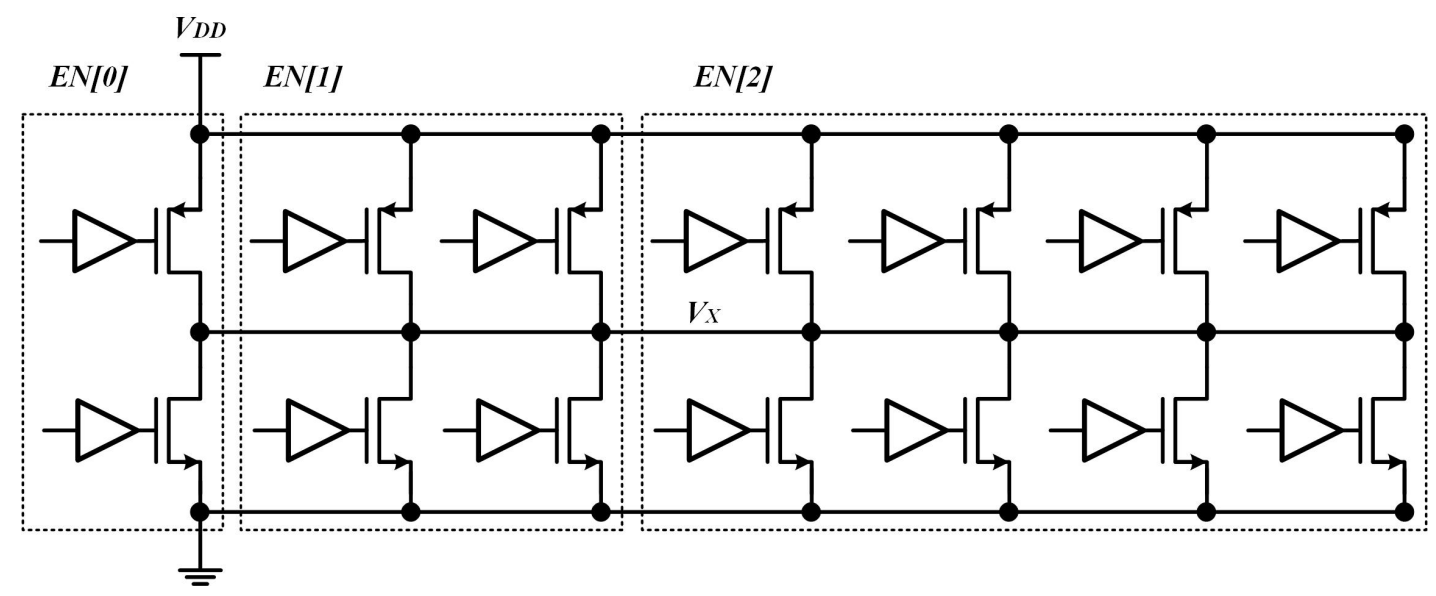

Figure 2.16: A circuit configuration showing the segmented output stage [4].

on-the-fly (as shown in Fig. 2.16). Since increasing the $W / L$ ratio can reduce the $R_{o n}$ of the MOSFET and subsequently reduce the conduction loss $P_{\text {cond }}$, enabling maximum output stage segments is beneficial at heavy load. On the other hand, observations have shown that the gate driver loss dominates the power loss at low current. An output stage with small transistor size exhibits an advantage in reducing $P_{s w}$ due to a low $C_{g s}$.

Research on segmented topology has been applied on the gate driver IC design to drive a practical IGBT by [22], and further developed on EMI mitigation by [7]. Figure 2.17 shows the general block diagram for the segmented gate driver circuits. Results show that the segmented gate driver has the ability to change the output resistance, which subsequently affects the switching speed, the switching node ringing and the input current oscillation. In addition, a gate driver with dynamic output resistance $R_{\text {out }}$ allows the suppression of the EMI noise without degrading the overall power conversion efficiency [7]. 


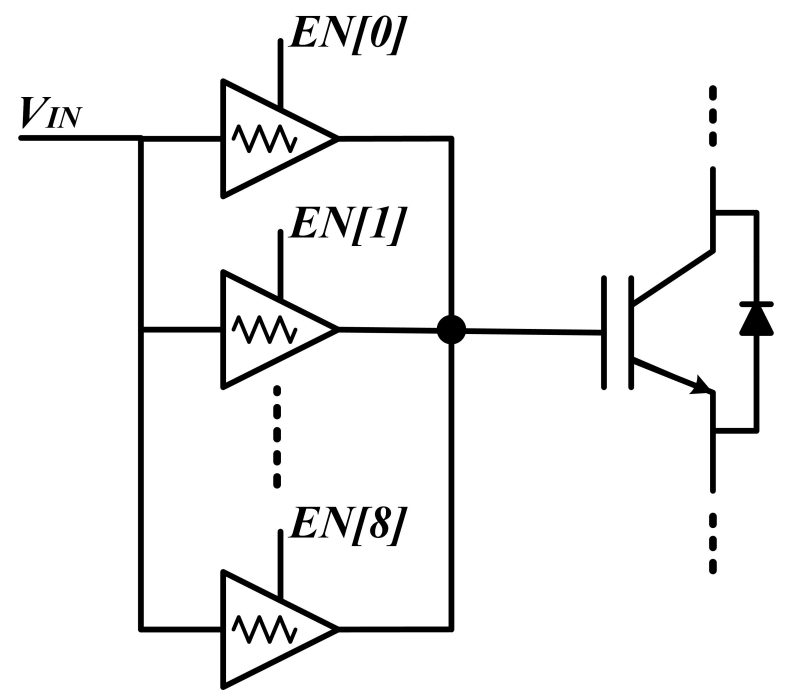

Figure 2.17: A simplified diagram for the segmented gate driver [22].

\subsection{Summary}

This chapter outlines the SMPS fundamentals, EMI in SMPSs, existing segmentation techniques employed in the integrated circuit. In Chapter 3, detailed design process for an H-bridge IC, which incorporates segmented output stages and segmented gate drivers, is presented. 


\section{Chapter 3}

\section{Segmentation Technique for the H-Bridge Design}

This chapter describes the segmentation technique used for the integrated H-bridge design. This feature allows the on-the-fly optimization of the power conversion efficiency over a wide range of load current. The influence of the power transistor's $R_{\text {on }}$ and the gate driver's $R_{\text {out }}$ on the power conversion efficiency is investigated via simulation. This is followed by a discussion on the optimization strategy.

\subsection{Influence of $R_{o n}$ of the Output Stage}

Based on the discussion in Section 2.2, the conduction loss in a DC-DC buck converter is dependent on the on-resistance $R_{o n}$ of the power transistor. Equation (2.6) shows that for a given output current, the conduction loss increases with $R_{o n}$, which is pre-determined by the width of the power transistor according to (2.7). Under heavy load conditions, where the conduction loss dominates the total power loss, an output stage with large size power transistors is preferred. However, a large power transistor in the output stage implies a large input capacitance $C_{g s}$, which requires more driving effort from the gate driver. It also consumes more power on charging and discharging the power transistors. An increase switching loss from the gate driver, as indicated by (2.10), degrades the overall power conversion efficiency at light load. The trade-off between small input gate capacitance $C_{g s}$ and small on-resistance $R_{o n}$ of the power transistors limits the performance of the traditional output stage over a wide range of load current.

In this section, a segmentation technique that allows the on-the-fly changes of the effective transistor size based on the load current condition is introduced. The high-side and low-side power devices are both $n$-type extended drain MOSFETs (EDMOS). Each 


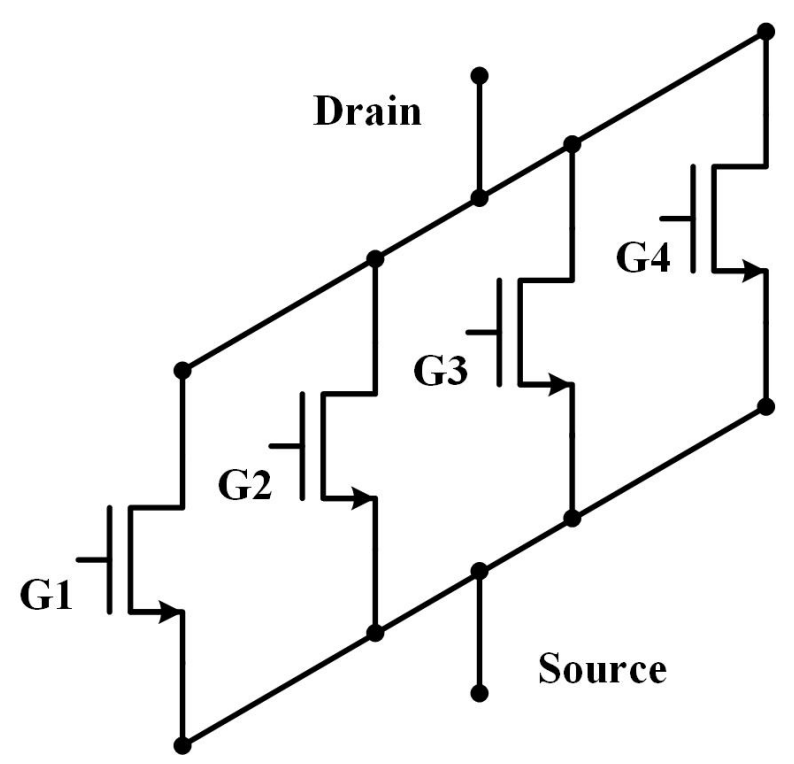

Figure 3.1: A segmented power transistor which contains four identical $n$-EDMOS devices.

Table 3.1: Characteristics of One $n$-EDMOS Segment

\begin{tabular}{c|c|c}
\hline Parameter & Value & Units \\
\hline \hline On-resistance & 26.6 & $\mathrm{~m} \Omega$ \\
\hline$C_{g s}$ & 230 & $\mathrm{pF}$ \\
\hline$C_{d b}$ & 7.7 & $\mathrm{nF}$ \\
\hline Threshold Voltage & 1.14 & $\mathrm{~V}$ \\
\hline Size $(W \times L)$ & $168,000 \times 1.3$ & $\mu m^{2}$ \\
\hline
\end{tabular}

power transistor is divided into 4 identical segments with independent gate drivers. As shown in Fig. 3.1, the four segments are connected in parallel: source and drain are shared, while four gates are controlled by its individual gate signals.

The simulated $R_{o n}$ of each EDMOS, is $26.6 \mathrm{~m} \Omega$. Table 3.1 lists the key characteristics of one $n$-EDMOS. This segmentation arrangement allows the entire power transistor to exhibit four possible effective on-resistance: $26.6 \mathrm{~m} \Omega, 12.8 \mathrm{~m} \Omega, 8.4 \mathrm{~m} \Omega$ and $6.3 \mathrm{~m} \Omega$ by enabling one to four segments, respectively.

In order to investigate the influence of $R_{o n}$ on the power conversion performance, a DC-DC buck converter as discussed in Section 2.1 with two proposed $n$-type segmented EDMOS transistors is simulated using HSpice. The circuit parameters are as listed in Table 3.2. The voltage and current waveforms at the input node and output node are monitored in order to evaluate the power conversion efficiency. 
Table 3.2: Buck Converter Testing Parameters for Different $R_{o n}$

\begin{tabular}{c|c|c}
\hline Parameter & Value & Units \\
\hline \hline Input Voltage $V_{D D}$ & 20 & $\mathrm{~V}$ \\
\hline Output Voltage $V_{\text {out }}$ & 4 & $\mathrm{~V}$ \\
\hline Load Current $I_{\text {out }}$ & $0.2-10$ & $\mathrm{~A}$ \\
\hline Switching Frequency $f_{s}$ & 500 & $\mathrm{kHz}$ \\
\hline Output Inductor $L$ & 47 & $\mu \mathrm{H}$ \\
\hline Output Capacitance $C_{\text {out }}$ & 2 & $\mu \mathrm{C}$ \\
\hline Gate Voltage $V_{G}$ & 5 & $\mathrm{~V}$ \\
\hline Enabled Output Stage Segment $(\mathrm{s})$ & 1 to 4 & - \\
\hline
\end{tabular}

Figure 3.2 shows the simulated power conversion efficiency curves for four different onresistance of the power transistor when current ranges from $0.1 \mathrm{~A}-10 \mathrm{~A}$. The gate driver loss and other fixed loss dominate the power loss at light load conditions. When the load current increases, the conversion efficiency first increases until it reaches a peak value. After that, the conduction loss dominates the power loss and degrades the conversion efficiency. From another perspective, at a particular load current, a specific output stage with appropriate effective $R_{o n}$ can maximize efficiency. At a low output current condition of $100 \mathrm{~mA}$, the output stage with only one segment enabled (Configuration A) achieves $4.2 \%$ efficiency improvement when compared to the all segments enabled situation (Configuration B). At a high output current of $10 \mathrm{~A}$, Configuration $B$ shows $7.6 \%$ improvement in yhe efficiency when compared to Configuration A.

\subsection{Influence of $R_{\text {out }}$ of the Gate Driver}

As discussed in Section 2.2, the switching loss is another main source of power loss in the DC-DC buck converter. Equation (2.9) shows that decreasing transition time $t_{o n}$, $t_{\text {off }}$ can help reduce the switching loss. According to (2.10), the gate driver loss is proportional to the input gate capacitance $C_{g s}$ of the power transistor. For a voltage driven strategy, as discussed in Section 2.6, the transition time is determined by two parameters: the output resistance $R_{\text {out }}$ of the gate driver and the input gate capacitance $C_{g s}$ of the power transistor. If a gate driver with small $R_{\text {out }}$ is used, the power transistor can be turned on and off fast so that switching loss is reduced. The observations above indicates that keeping $R_{\text {out }}$ as small as possible is an ideal approach for achieving high 


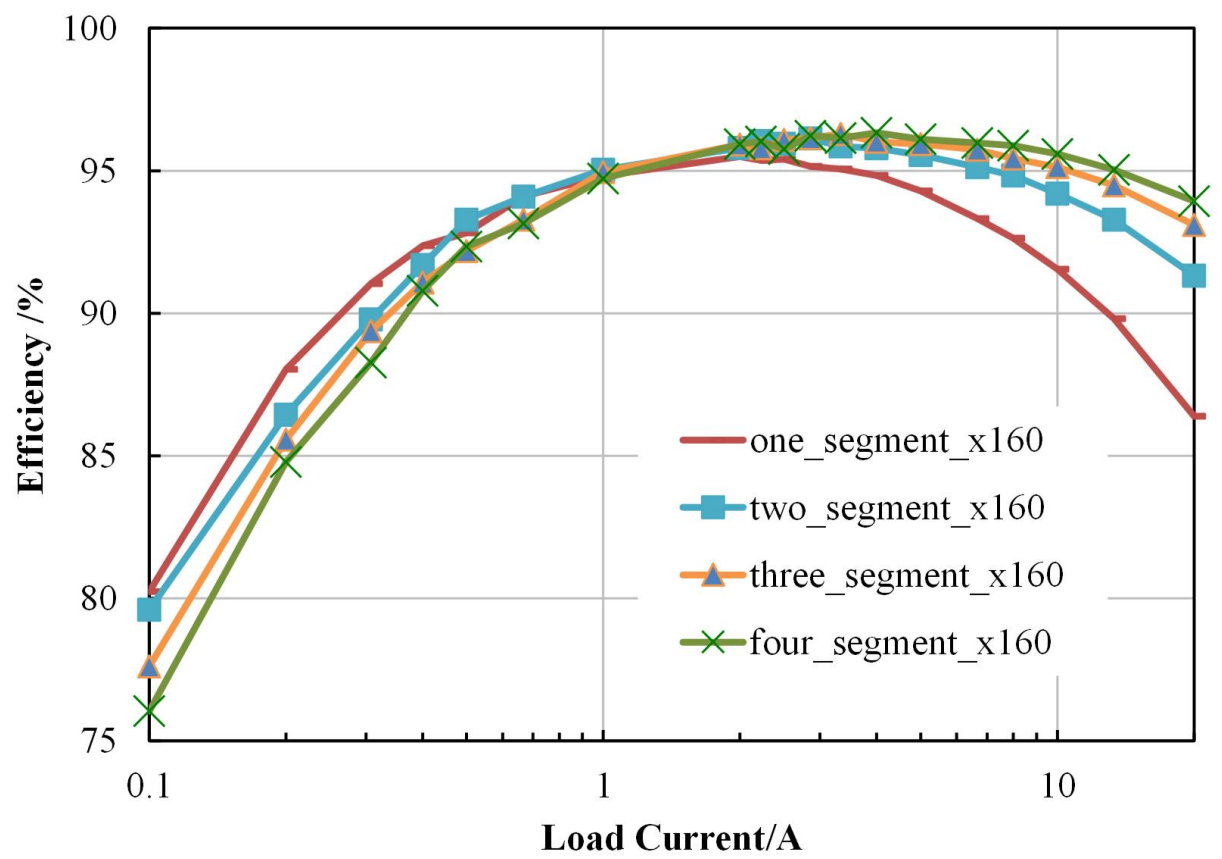

Figure 3.2: Efficiency versus load current for different enabled output stage segments.

conversion efficiency when delivering large output current.

However, as mentioned in Section 2.4, the $R_{\text {out }}$ of the gate driver, the parasitic inductance existing on the path from gate driver to the gate node of the power transistor, and the $C_{g s}$ of the power transistor together forms a RLC circuit. Depending on the values of $R_{\text {out }}, L$ and $C_{g s}$, ringing oscillation could appear at the gate node of the power transistor. Figure 3.3 shows a simple equivalent RLC circuit [24]. Equations (3.1), (3.2) and (3.3) are the mathematical expressions of this second-order system. The damping factor $\zeta$ is proportional to the output resistance. A small $R_{\text {out }}$ for the gate driver exhibits fast rise and fall times, but leads to larger ringing at the gate node, which is subsequently amplified at the switching node of the DC-DC buck converter. The discussion above explains the phenomenon that CEMI occurs during the switching transition.

$$
\begin{gathered}
g(s)=\frac{1}{s^{2} L C+s R C+1} \\
\zeta=\frac{R}{2} \sqrt{\frac{L}{C}} \\
\omega_{n}=\frac{1}{\sqrt{L C}}
\end{gathered}
$$




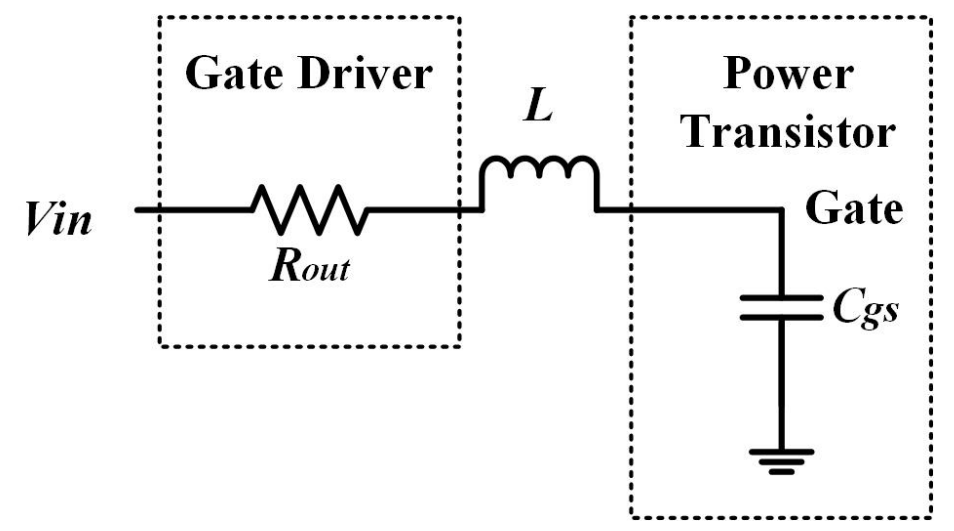

Figure 3.3: Equivalent RLC circuit for the gate driver.

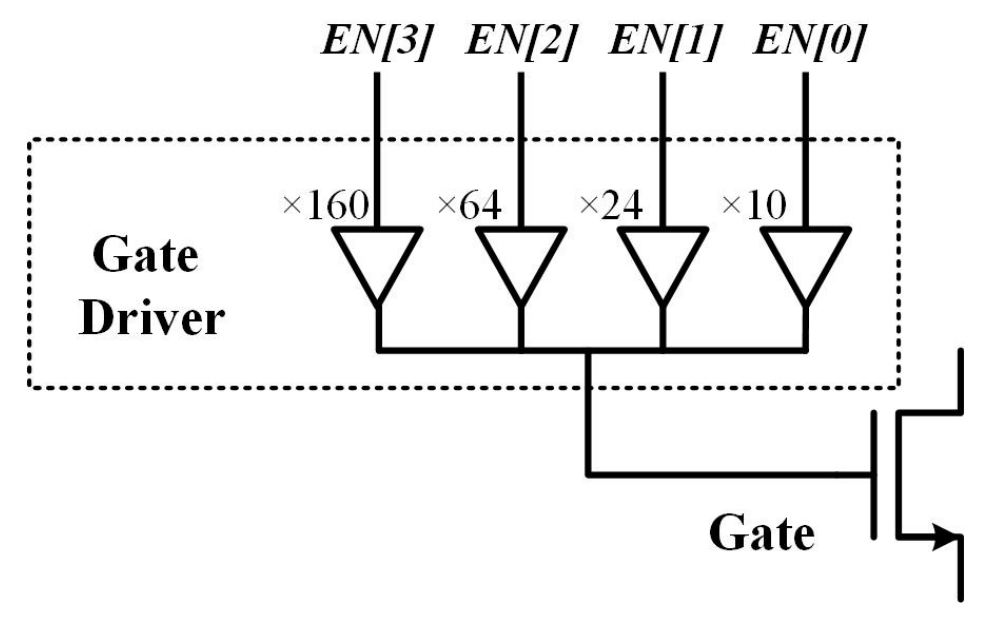

Figure 3.4: Segmented gate driver design.

In [7], the authors proposed a driving strategy to dynamically adjust the driving strength of the gate driver during the switching of the power MOSFET. Experimental results proved that by using low $R_{\text {out }}$ during the transition and then switching to large $R_{\text {out }}$ can effectively balance the trade-off between fast switching speed and low EMI. Low output resistance allows the gate node of the power transistor to be pulled up and down in a short time. In the second stage, high $R_{\text {out }}$ is selected to over-damp the ringing and overshoot, which help to eliminate CEMI before it is generated.

In this thesis, an integrated segmented gate driver is designed to drive an on-chip $n$-type EDMOS. The gate driver proposed here has a push-pull complementary design, which has a $p$-type MOSFET on the high-side and an $n$-type MOSFET on the low-side. Each gate driver is divided into 4 segments with size ratios of 10:24:64:160. As shown in Fig. 3.4, the four gate driver segments are connected in parallel similar to the segmented output stage design. Each segment has independent enable signal at the input node. The sizing factors are $\times 10, \times 24, \times 64$, and $\times 160$, for each gate driver segment. 
Table 3.3: Buck Converter Testing Parameters for Different $R_{\text {out }}$

\begin{tabular}{c|c|c}
\hline Parameter & Value & Units \\
\hline \hline Input Voltage $V_{D D}$ & 20 & $\mathrm{~V}$ \\
\hline Output Voltage $V_{\text {out }}$ & 4 & $\mathrm{~V}$ \\
\hline Load Current $I_{\text {out }}$ & $0.2-10$ & $\mathrm{~A}$ \\
\hline Switching Frequency $f_{s}$ & 500 & $\mathrm{kHz}$ \\
\hline Output Inductor $L$ & 47 & $\mu \mathrm{H}$ \\
\hline Output Capacitance $C_{\text {out }}$ & 2 & $\mu \mathrm{C}$ \\
\hline Gate Voltage $V_{G}$ & 5 & $\mathrm{~V}$ \\
\hline Parasitic Inductances $L_{v d d}, L_{g n d}, L_{v x}$ & 500 & $\mathrm{pF}$ \\
\hline Enabled Output Stage Segment $(\mathrm{s})$ & 1 & - \\
\hline Gate Driver Sizing Factor & $\times 24, \times 64, \times 160, \times 258$ & - \\
\hline
\end{tabular}

In order to investigate the impact of the driving strength (inversely proportional to the $R_{\text {out }}$ ) of the gate driver on the power conversion efficiency, the same buck converter circuit described in Section 3.1 is studied using HSpice. The operating condition is as listed in Table 3.3. The power conversion efficiencies for different driving strength over a range of load current are as shown in Fig. 3.5. For a given power transistor size and gate driving strength, the transition period $\left(t_{o n}\right.$ and $\left.t_{\text {off }}\right)$ is fixed. According to (2.9), the MOSFET switching loss increases when load current becomes larger. A gate driver with low $C_{g s}$ is preferred in this case. Nevertheless, a gate driver with high driving strength implies a large output stage, which requires a long inverter chain. The inverter chain consumes power for charging and discharging of the internal capacitance, which is independent of the load current according to (2.15). Under light load conditions, this gate driver power loss exceeds the other losses and degrades the overall efficiency. Figure 3.5 shows that the buck converter circuit with low driving strength exhibits $4.4 \%$ higher power conversion efficiency when compared to the one with strong driving strength.

\subsection{Optimized Operating Strategy of H-bridge}

Traditional H-bridge designs are optimized to achieve high power conversion efficiency for a specific set of operating conditions, such as switching frequency, conversion ratio and load current. Such design greatly limits the practical applications of the H-bridge IC, since a constant, unchanging operating condition is not common. Simulation results 


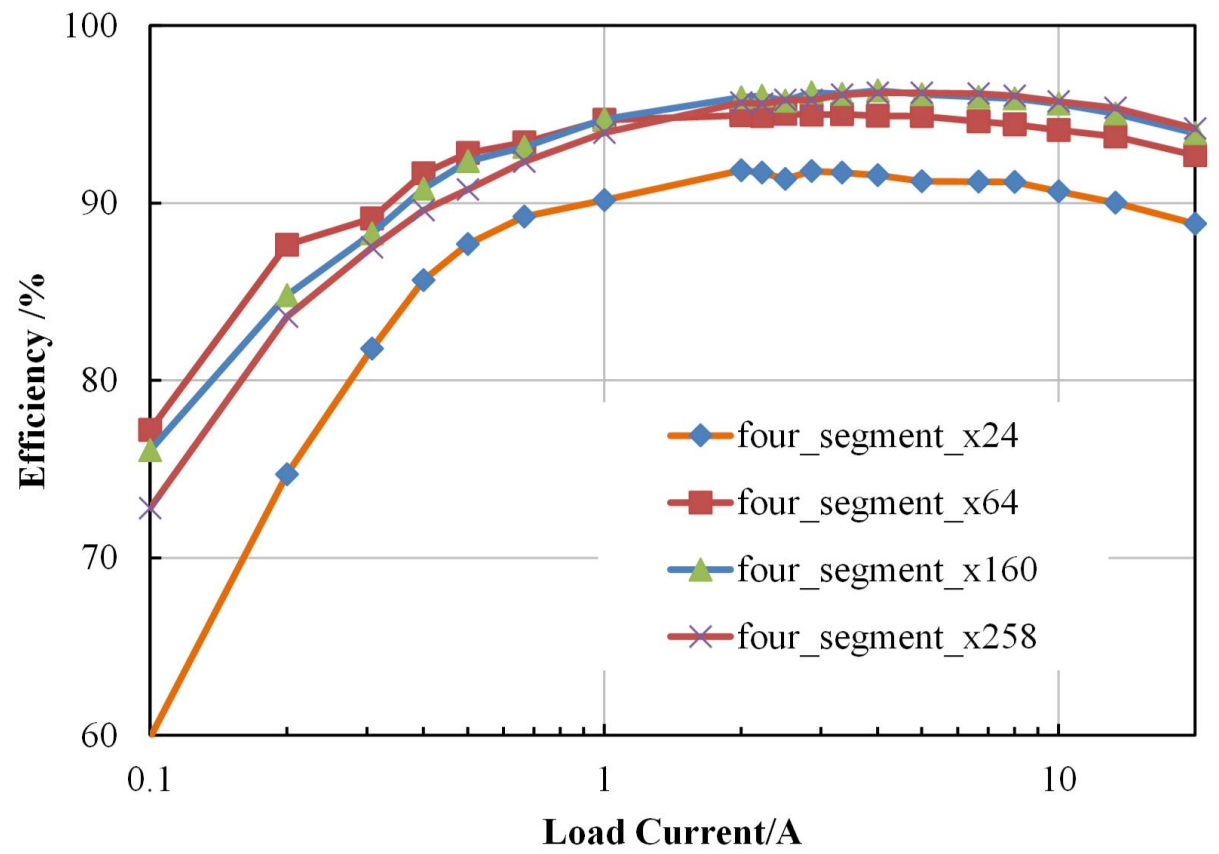

Figure 3.5: Efficiency versus load current for different gate driving strengths.

Table 3.4: Proposed Operating Strategy

\begin{tabular}{|c|c|c|c|}
\hline \multirow{2}{*}{\multicolumn{2}{|c|}{$\begin{array}{c}\text { Load Current } \\
I_{\text {out }} \\
\end{array}$}} & \multicolumn{2}{|c|}{ Output Stage Size } \\
\hline & & Small & $B i g$ \\
\hline \multirow{2}{*}{$\begin{array}{c}\text { Gate Driver } \\
R_{\text {out }}\end{array}$} & High & Light & - \\
\hline & Low & - & Heavy \\
\hline
\end{tabular}

and analysis from Sections 3.1 and 3.2 show that if the load current changes, the output stage power transistor and the gate driver should be changed accordingly in order to continuously maintain the optimal power conversion efficiency.

The proposed segmentation technique for both the output stage and the gate drivers is one way to achieve this functionality. For operation at high load current, all segments of the output stage are enabled and the gate drivers also operate with all segments enabled, minimizing the gate driver output resistance. For operation with low load current, a single segment of the output stage is enabled and the gate drivers operate with only one single segment is to maximize the gate driver output resistance. The proposed operating strategy is summarized in Table 3.4.

The high load current strategy outlined above utilizes a very low $R_{\text {out }}$ for gate drivers. Unfortunately, a low gate driver $R_{\text {out }}$ can lead to excessive ringing at the gate node of the transistor, which subsequently produces ringing at the switching nodes of the H-bridge. 


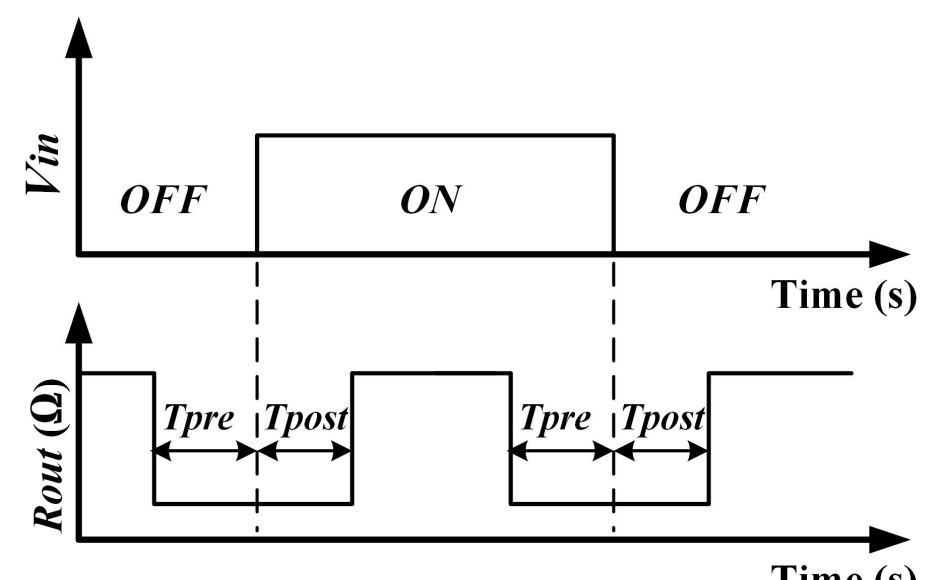

Figure 3.6: Timing diagram for the driving strategy.

Therefore, this operating strategy can potentially generate excessive CEMI. In order to minimize ringing at the switching node, an operation similar to the one proposed in [7] is employed. The gate driver output resistance $R_{\text {out }}$ is set high for a short period while the power transistor turns on and off. The dynamic driving strategy is shown in Fig. 3.6. As the high-side gate receives a switching signal, $R_{\text {out }}$ is first set to be low to decrease the rise-time and then set to be high after the high-side gate is turned on. In this process, two critical timing $T_{\text {pre }}$ and $T_{\text {post }}$ require careful tuning based on different gate strength combinations.

\subsection{System Topologies and Schematic Design}

This section described the system topologies and the detailed schematic design of two important functional blocks.

\subsubsection{System Topologies}

In order to verify the proposed optimization operating strategy as outlined in the previous section, a segmented H-bridge IC is designed as shown in Fig. 3.7.

As mentioned in Section 3.1, each power device of the proposed segmented output stage is equally partitioned into four identical segments. Figure 3.8 illustrates the arrangements for the power transistors and the gate drivers. For each independent tunable gate driver, four parallel output stages are designed with size ratio of 10:24:64:160.

In order to enable every possible combinations of segmented output stage and tunable gate driver, a digital circuit which stores the digital control code is also integrated, as 


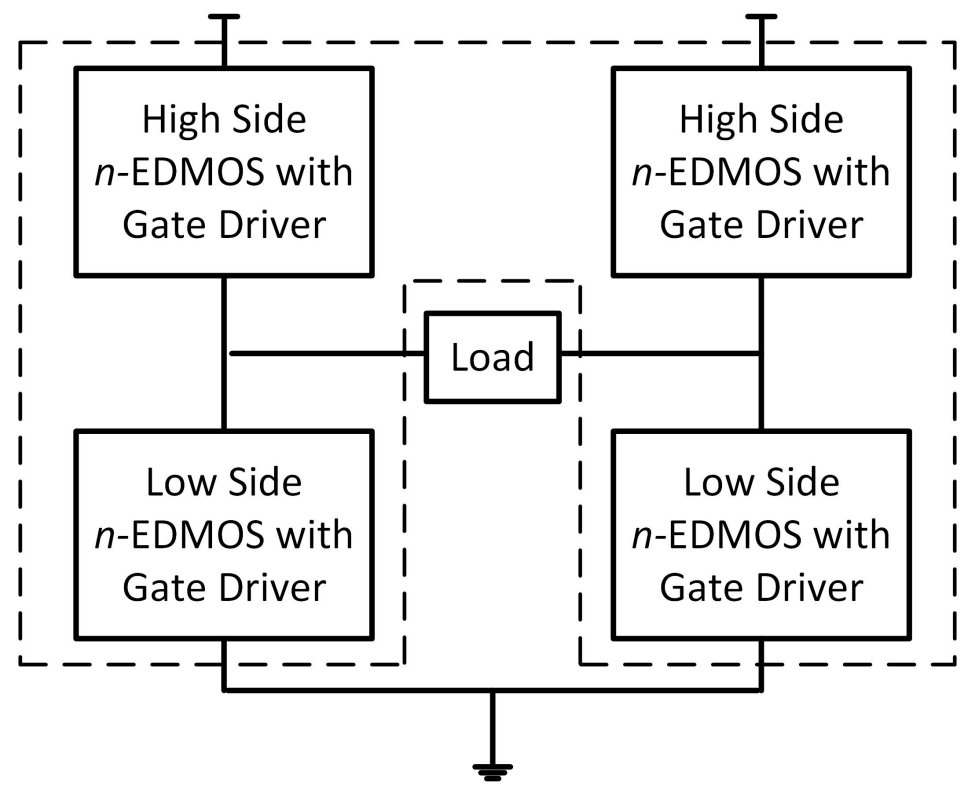

Figure 3.7: Block Diagram of the Integrated Power H-bridge.

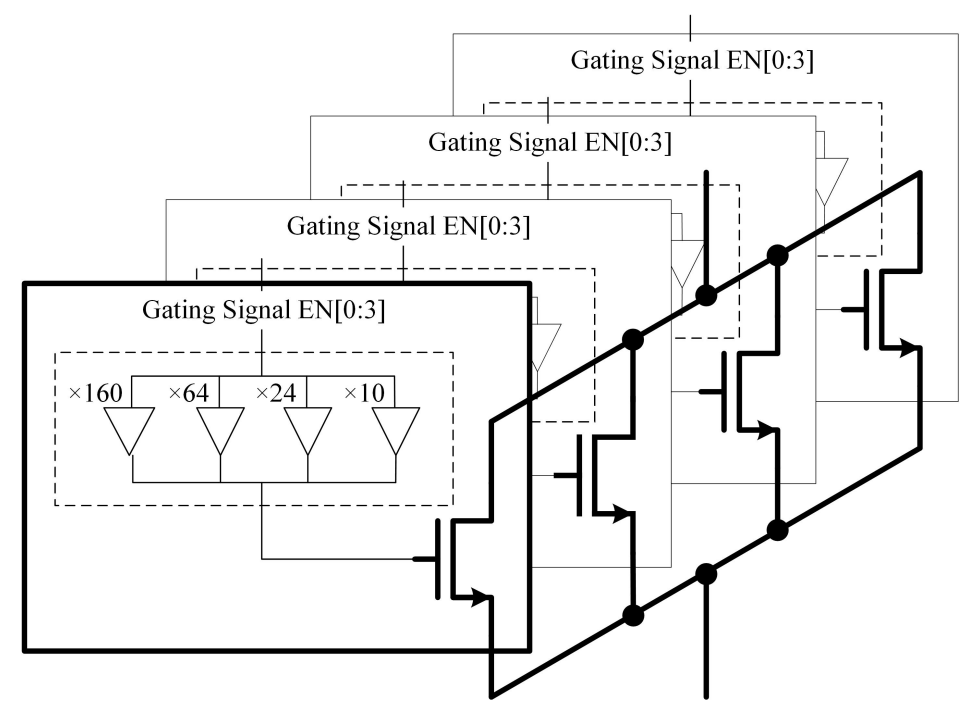

Figure 3.8: Block diagram of one $n$-EDMOS with segmented gate driver arrangement. 


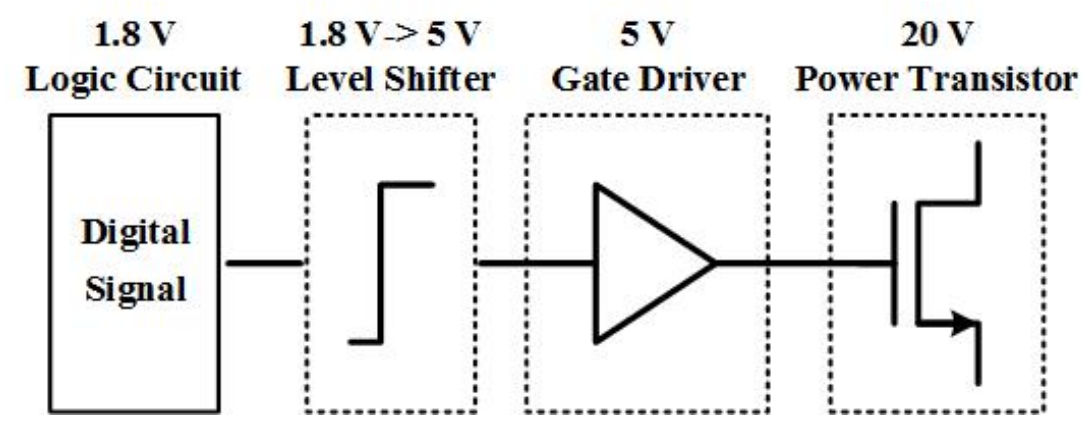

Figure 3.9: Block diagram for driving the low-side $n$-EDMOS transistor.

shown in Fig. 3.9. The digital circuits utilizes $1.8 \mathrm{~V}$ low voltage device. A level shifter circuit is required to convert the $1.8 \mathrm{~V}$ digital signal to a $5 \mathrm{~V}$ signal.

\subsubsection{Schematic Design}

\section{Digital Data Shifting}

In order to digitally control the behavior of every gate driver segments and output stage segment, the control signal is shifted through the entire chip serially to decrease the pin-count requirement.

Normally, a shifted register circuit containing a chain of D flip-flops with an reset option, as shown in Fig. 3.10, can be used. A potential problem exist, in which the serial data and clock signal propagate in the same direction, as shown in Fig. 3.11. At time $t_{0}$, $D A T A[0]$ is shifted to node $D 1$, and the clock signal propagates to node $C 1 . t_{D C}$ and $t_{D D}$ refer to the delay times for the $\mathrm{D}$ flip-flop and the buffer, respectively. If $t_{D C}$ is longer than $t_{D D}, D A T A[0]$ is shifted to node D2 at time $t_{2}$. This situation is unwanted, since at time $t_{2}$, D2 is supposed to hold the data from last clock cycle. This shifting error may occur in every clock cycle.

This problem can be solved by shifting the digital signal and the triggering clock signal from two ends of the D flip-flop chain. Figure 3.12 shows the unit cell schematic of control signal shifting circuit. The state select signal $S E L$ is used to enable the output code $E N[0: 3]$ to adjust $R_{\text {out }}$ on-the-fly. Two states can be dynamically switched using this topology.

\section{Segmented Gate Driver}

Figure 3.13 shows the general schematic for one segment the of gate driver circuit. It contains an non-overlap circuit and a pull-push complementary output stage. The nonoverlap circuit is designed to minimize the overshoot current of the output stage. 


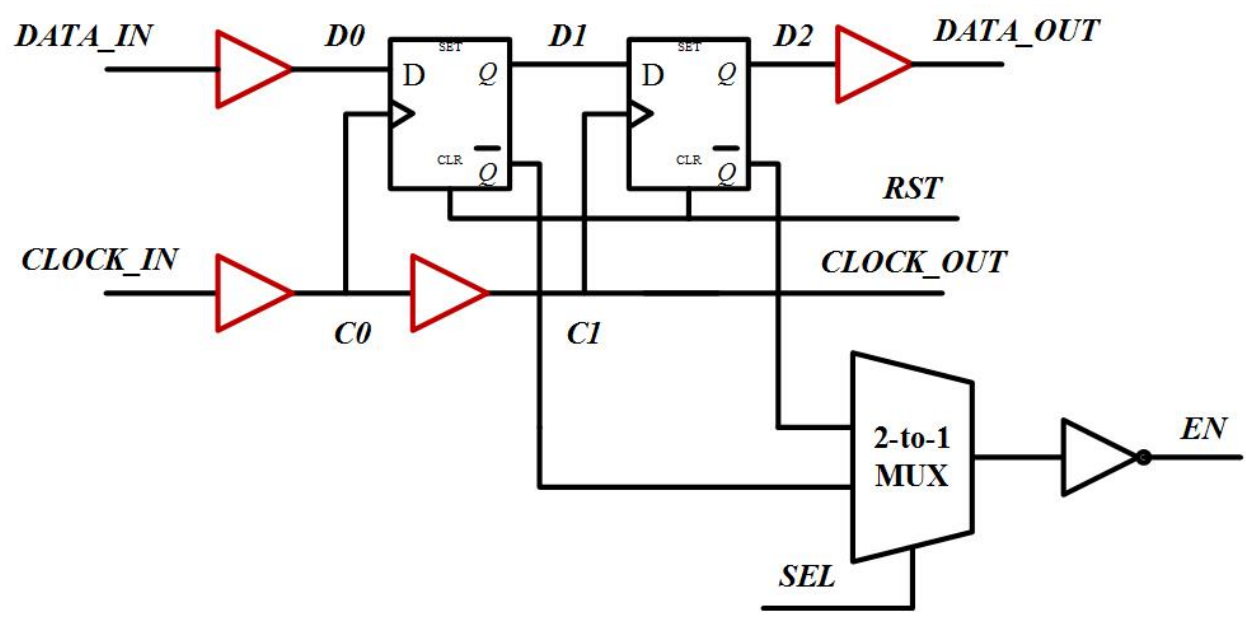

Figure 3.10: Data shift in the same direction as the clock signal.

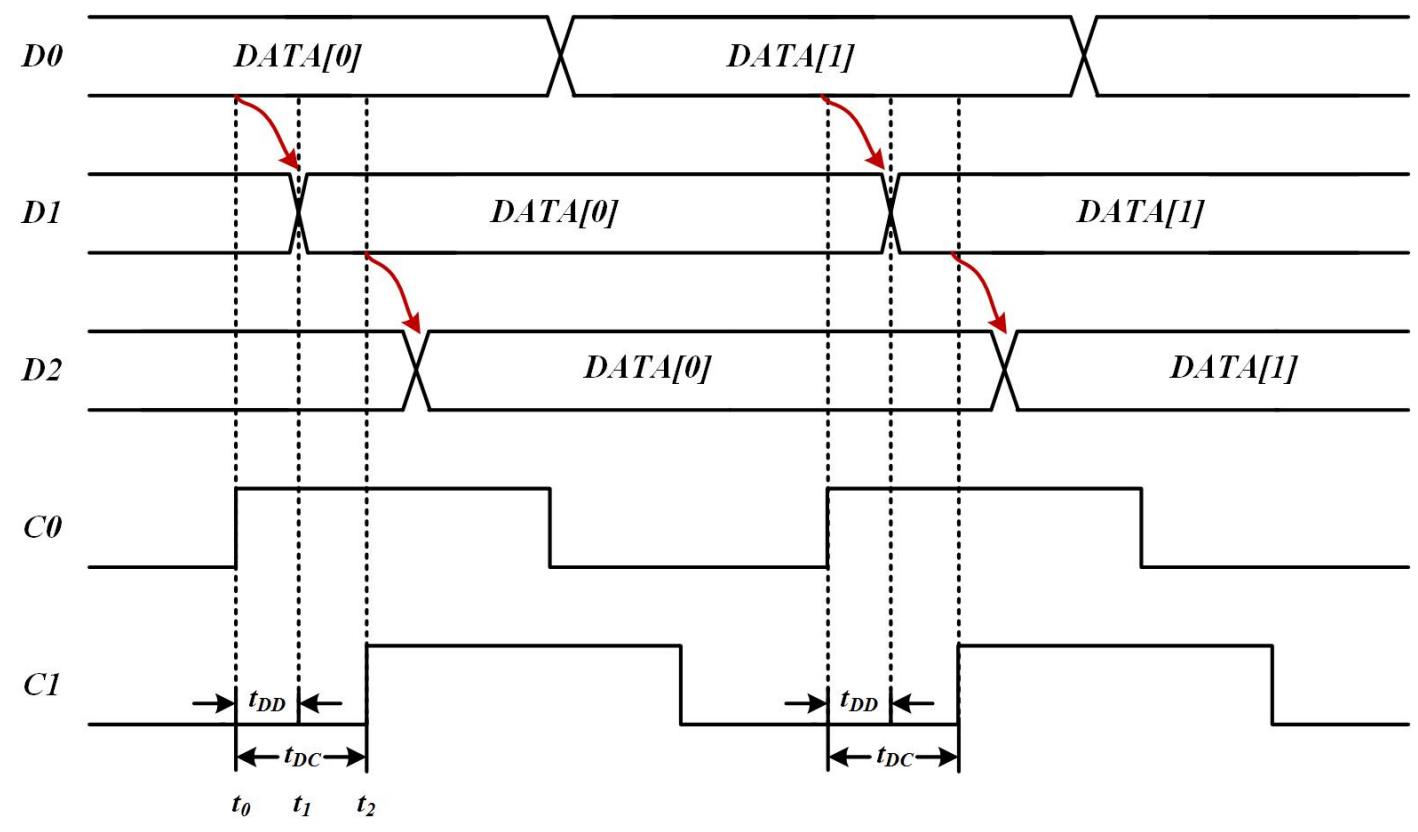

Figure 3.11: Hold time problem. 


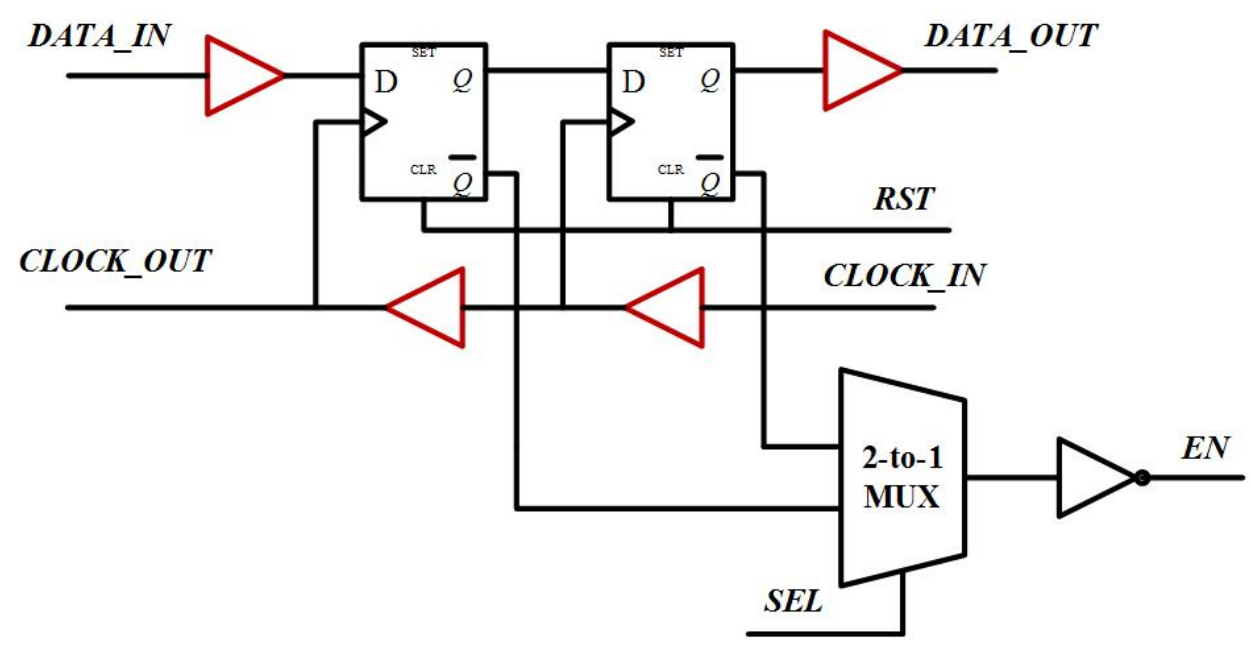

Figure 3.12: Unit cell of the data shifting circuit.

Input signals $D, E N[0: 3]$ and $I N I T$ are used to flexibly enable or disable the output stage of one gate driver segment. $D$ is an external gating signal which determines the behavior of the gate driver for one power transistor segment. The EN[0:3] and INIT signals are generated by the digital circuit and then converted to the required $5 \mathrm{~V}$ level. If a segment of the gate driver is enabled, the resistance $R_{\text {out }}$ seen at the output node is determined by the size of the gate driver output stage size. If a segment is disabled, the resistance seen at the output node exhibits high impedances and the $R_{\text {out }}$ of the entire gate driver is dependent on how many segments are enabled.

There is a special condition when all the gate driver segments received a zero EN signal, which makes the final $R_{\text {out }}$ shows high impedance. This is an unwanted situation during operation, since the floating output voltage of the gate driver leads to unpredictable power MOSFET states. Therefore, a initialize signal INIT is used to control the gate driver behavior under such condition. When $E N[0: 3]$ is [0000], INIT is set to 0 , in which case the low-side transistor in the output stage of the gate driver is forced to be on. This means the voltage at the output node of the gate driver is low, so the connected power device can be guaranteed to be off. INIT is set to high except for the situation when $E N[0: 3]$ is [0000]. Table 3.5 illustrates the outcome for different input signal conditions.

\subsection{Summary}

In this chapter, the influence of the power transistor $R_{o n}$ and the gate driver $R_{\text {out }}$ on the power conversion efficiency is examined by simulation. An operating strategy is to 


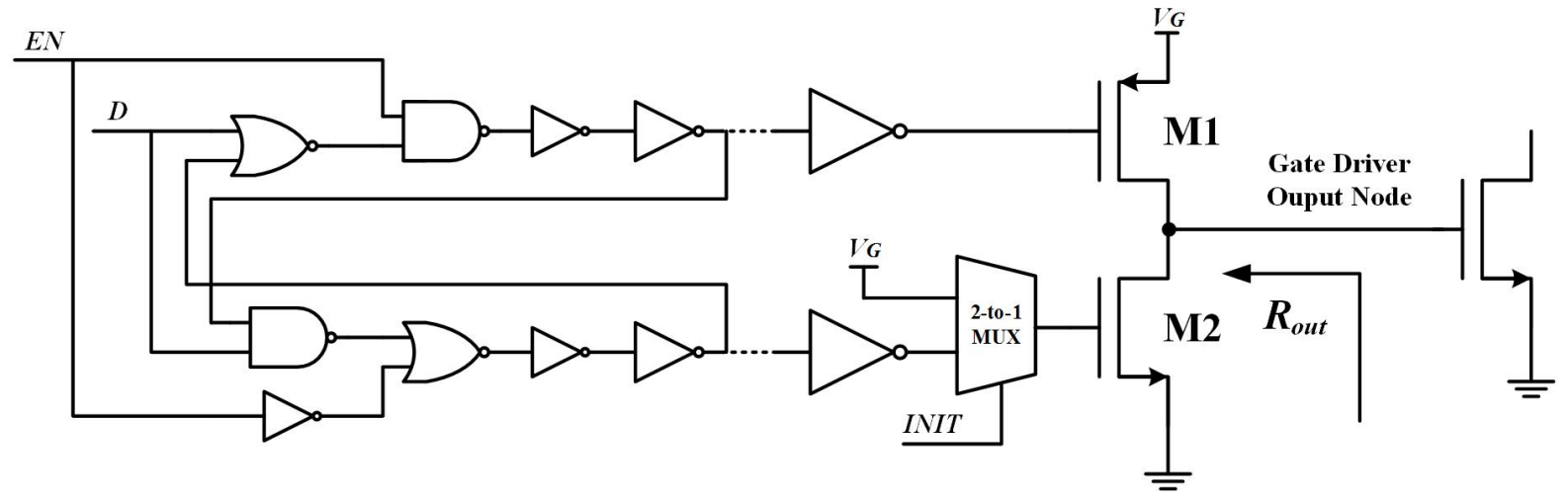

Figure 3.13: General schematic of each gate driver segment.

Table 3.5: Gate Driver Segment Behaviors.

\begin{tabular}{c|c||c|c}
\hline \multicolumn{2}{c||}{$\begin{array}{c}\text { Segment } \\
\text { Behavior }\end{array}$} & \multicolumn{2}{c}{$\mathrm{D}$} \\
\cline { 3 - 4 } Enable Signal & $\boldsymbol{0}$ & High $Z$ & High $Z$ \\
\cline { 2 - 4 }$E N$ & $\mathbf{1}$ & off & on \\
\hline INIT $=\mathbf{0}$ & \multicolumn{2}{c}{ off } \\
\hline
\end{tabular}

allow the continuous optimization of the power conversion efficiency over a wide range of load current. In Chapter 4, the implementation and experimental measurements are presented. 


\section{Chapter 4}

\section{Implementation and Experimental Results}

This chapter outlines the IC implementation of the proposed H-bridge IC design, and the experimental measurement in practical circuit applications. Layout design for the implementation of the segmentation technique is provided. The experimental setup is then described. Finally, measurement results for the proposed operating strategy from two aspects, namely power conversion efficiency optimization and EMI reduction, are provided.

\subsection{IC Layout Design and Package Specifications}

The proposed segmented H-bridge IC is fabricated using TSMC's $0.18 \mu \mathrm{m}$ BCD Gen-2 process technology. 1 layer of polysilicon and 5 layers of metal with the ultra-thick top metal back-end technology are utilized in fabrication. The micrograph in Fig. 4.1 shows the full layout of the H-bridge IC. The chip occupies an area of $5 \mathrm{~mm} \times 5 \mathrm{~mm}$. Two identical output stages with independent input voltage nodes are placed symmetrical to each other on two sides of the chip. The low voltage digital circuit and the segmented gate drivers are located in the middle. Table 4.1 shows the area requirement for the primary functional blocks.

A simple diagram of the segmented power transistor layout pattern is shown in Fig. 4.2. Each segment of the power transistor, represented by different number, is placed around a common centroid pattern to avoid mismatch and improve linearity. The segmented gate drivers are designed with a similar layout pattern.

The final IC is mounted into a LCC84 package, as shown in Fig. 4.3 and 4.4. Table 4.2 shows the specifications for this package. 


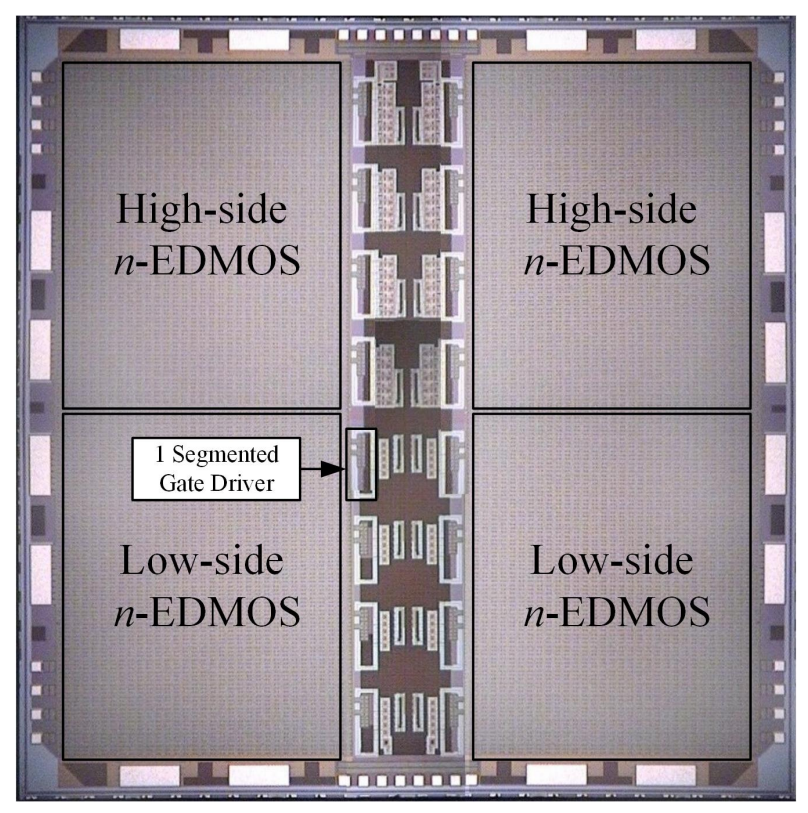

Figure 4.1: A micrograph of the full layout for the H-bridge IC.

Table 4.1: Area Requirement for Primary Functional Blocks.

\begin{tabular}{c|c}
\hline Parameter & Area $\left(\mathbf{m m}^{2}\right)$ \\
\hline \hline Output Stages & 17.78 \\
\hline Gate Drivers & 0.87 \\
\hline Level Shifters & 0.77 \\
\hline Digital Circuit & 0.19 \\
\hline
\end{tabular}

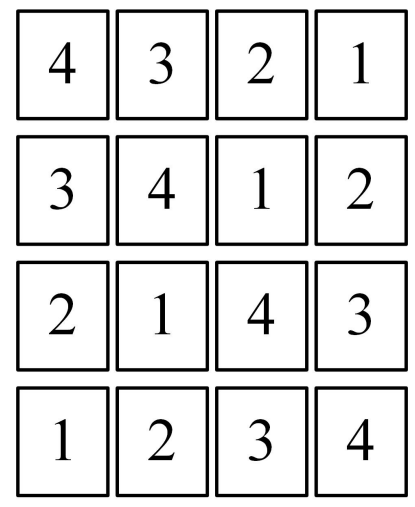

Figure 4.2: Common centroid layout pattern for one power transistor. 


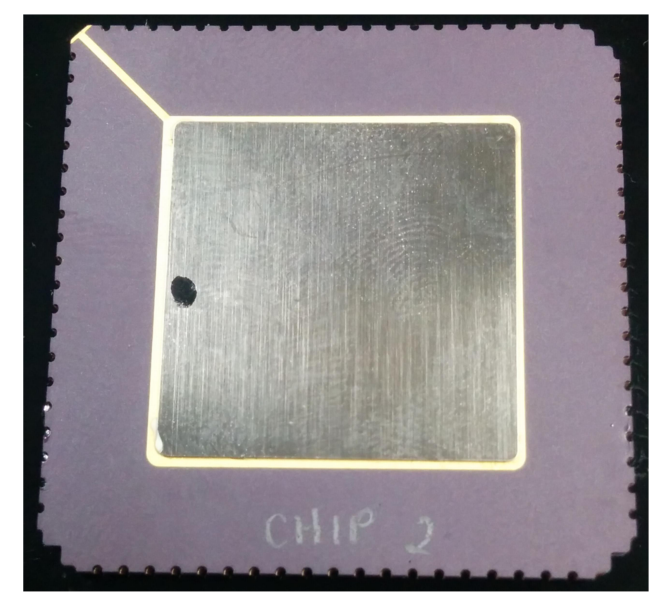

Figure 4.3: H-bridge IC mounted in the LCC84 package.

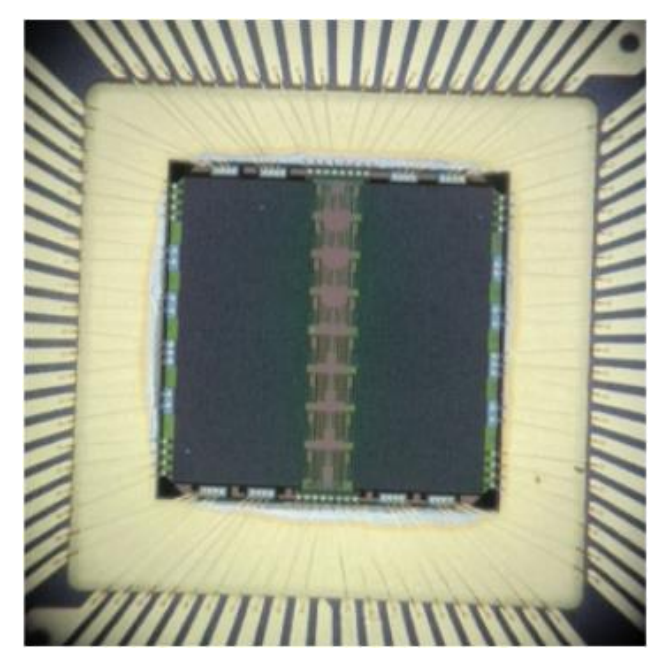

Figure 4.4: Micrograph showing the wire bonding inside the LCC84 package.

Table 4.2: LCC84 Package Specifications.

\begin{tabular}{c|c}
\hline Item & Description/Specification \\
\hline \hline Package Type & Leadless Ceramic Carrier (LCC) \\
\hline Bare Die Size & $5 \mathrm{~mm} \times 5 \mathrm{~mm}$ \\
\hline Pin Number & 84 \\
\hline Bonding Wire Material & $\mathrm{Au}$ \\
\hline Bonding Wire Diameter & $1.3 \mathrm{mil}$ \\
\hline Wire Max Current & $1 \mathrm{~A}$ \\
\hline Pad Pitch & $0.05 \mathrm{mil}$ \\
\hline HV Pad Number & 16 \\
\hline LV I/O Number & 32 \\
\hline
\end{tabular}




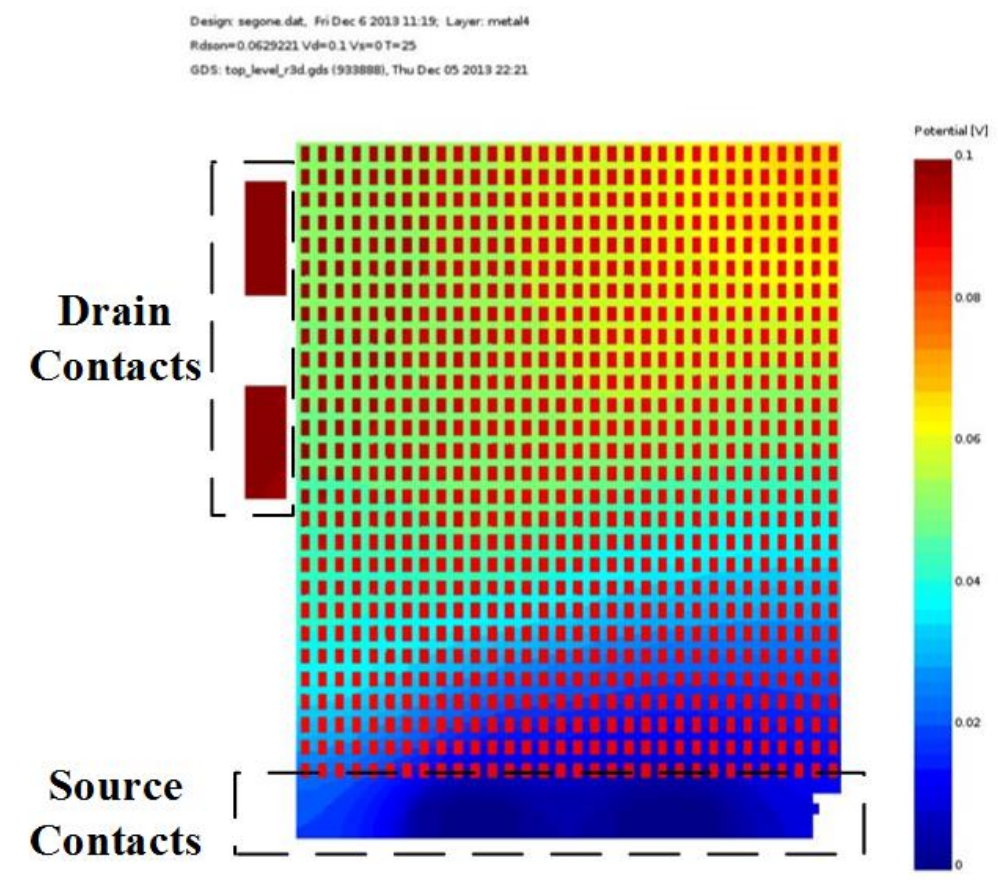

Figure 4.5: Voltage potentials on Metal 4 layer as simulated using $R 3 D$.

\subsection{On-resistance $R_{o n}$ Measurements}

In order to verify the segmented output stage design, the power transistor's on-resistance $R_{o n}$ is simulated or measured. The ideal Spectre model only incorporates the channel resistance. It grossly under-estimate the on-resistance of the power transistor. The postlayout resistance extracted from $R 3 D$ takes metal interconnect resistance into account, but not the bond wire resistance. The actual $R_{\text {on }}$ was measured on the PCB after chip fabrication.

$R 3 D$ is a novel software which can extract the post-layout $R_{\text {on }}$ of power semiconductor devices [25]. In addition, $R 3 D$ provides a graphical user interface to display the analytical results such as the current density and the voltage potentials. It allows the users to customize the parameters, i.e. technology process, bond wire contacts location and related parasitic resistance, which help build a realistic model and makes the extracted resistance convincing [25].

Figures 4.5 and 4.6 demonstrates the graphical $R 3 D$ results for only one segment of the power transistor enabled situation. The potential distribution and current density profile are simulated and monitored in these two figures, respectively. Figure 4.7 shows a report of the post-layout resistance extraction.

The simplified representation of the experimental setup to measure the $R_{o n}$ is as shown in Fig. 4.8. Since the two power transistors in the output stage are identical, 
Design segone dat, Fri Dec 620131119 , Layer: Offution
Rdson $=0.0629221$ Vd $=0.1$ Vs $=0 \mathrm{~T}=2 \mathrm{~S}$

GDS: top_level_r3d gds (933888), Thu Dec 05 20132222

\section{Enabled}

Segment

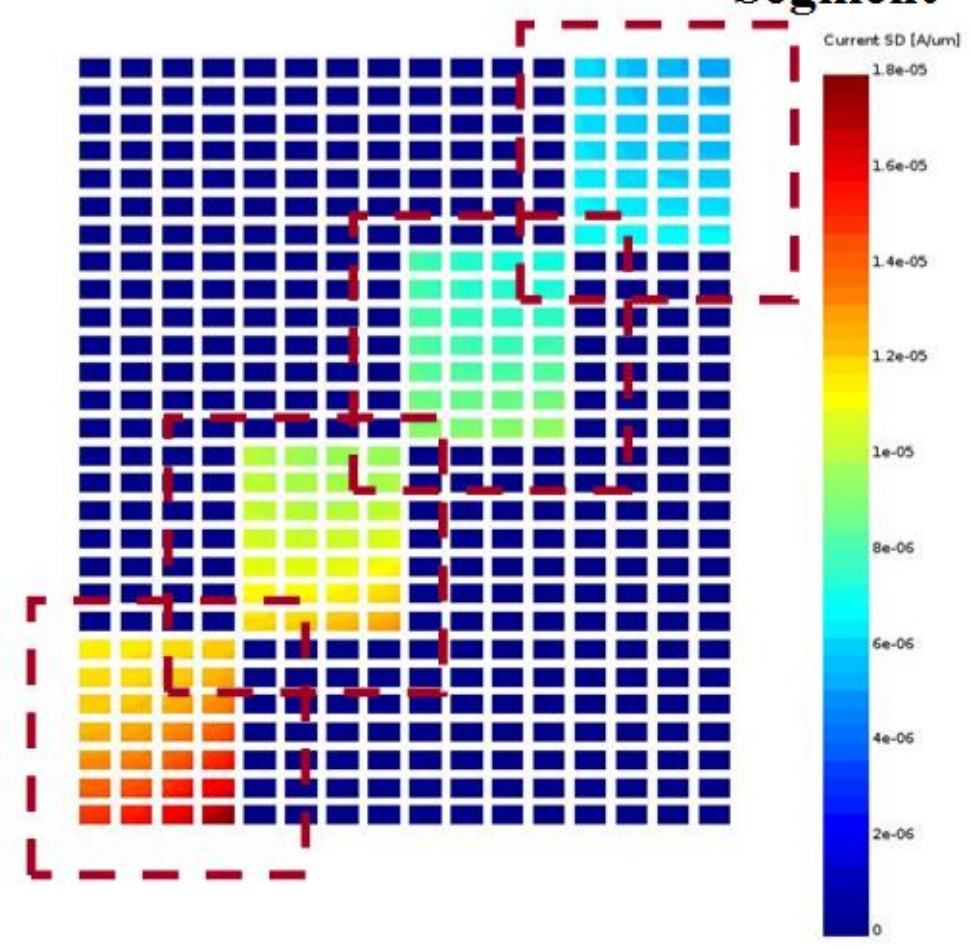

Figure 4.6: Current density on the diffusion region as simulated using $R 3 D$.

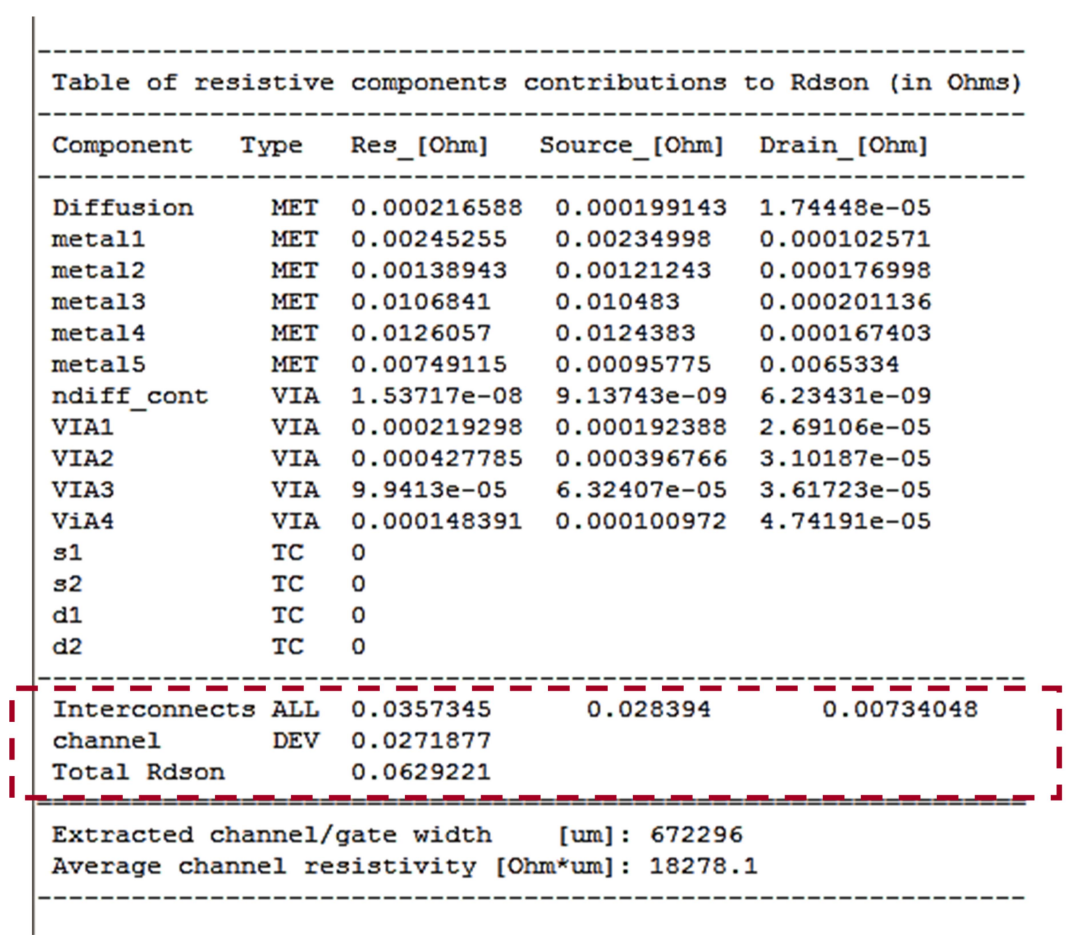

Figure 4.7: Post-layout resistance extraction report generated from $R 3 D$ simulation. 
Table 4.3: Summary of $R_{\text {on }}$ Measurements.

\begin{tabular}{c||c|c|c|c}
\hline \multicolumn{1}{c||}{\multirow{2}{*}{$\begin{array}{c}R_{\text {on }} \\
(\mathrm{m} \Omega)\end{array}$}} & \multicolumn{4}{c}{ Enabled Segment(s) } \\
\cline { 2 - 5 } & $\mathbf{1}$ & $\mathbf{2}$ & $\mathbf{3}$ & $\mathbf{4}$ \\
\hline \hline Spectre & 26.6 & 12.8 & 8.4 & 6.3 \\
\hline $\boldsymbol{R} 3 \boldsymbol{D}$ & 62.9 & 45.9 & 38.1 & 34.9 \\
\hline Measured & 134 & 118 & 111 & 108 \\
\hline
\end{tabular}

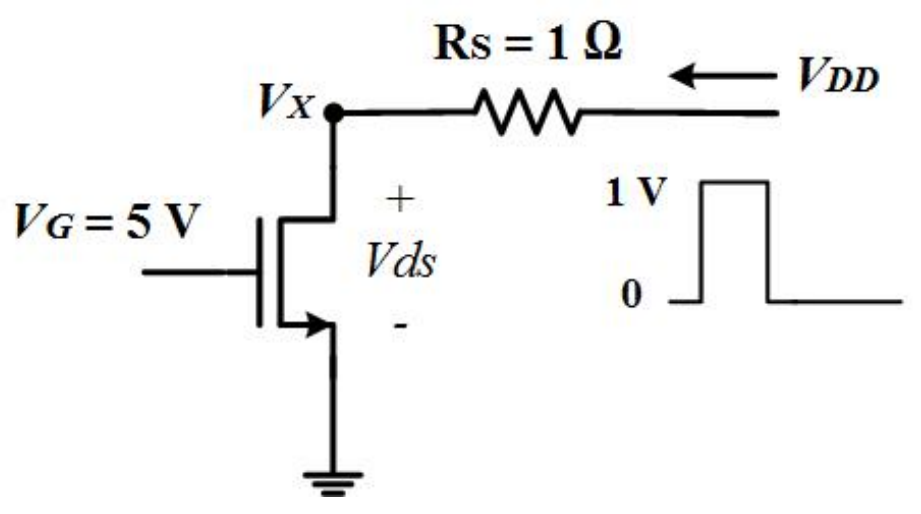

Figure 4.8: Simplified circuit diagram for $R_{o n}$ measurement.

the $R_{o n}$ measurement here only focus on the low-side transistor. The high-side power transistor is disabled and the low-side one is turned on. A $1 \Omega$ power resistor is connected to the switching node of the original output stage. It is well known that MOSFETs have a positive temperature coefficient. If the low-side $n$-EDMOS is conducting a high current for a long time, the $R_{o n}$ goes higher. Therefore, a $1 \mathrm{~V}$ voltage pulse is applied at the input node to avoid such heat effect. $V_{d s}$ is measured for different number of enabled segments. The on-resistance $R_{o n}$ is calculated by dividing $V_{d s}$ over the current. Table 4.3 lists the results obtained by different models and testing situations. The comparison indicates that for each segment of the power transistor, five layers of metal bring about 25 to $36 \mathrm{~m} \Omega$ to the ideal channel resistance, while bonding wires from a practical package and PCB traces bring another $70 \mathrm{~m} \Omega$ parasitic resistance. Metal routing in the layout design further increases to the total $R_{o n}$. The parasitic resistance is fixed for a given package and PCB. Its influence on the total resistance increases when all segments of the power transistor are enabled. 


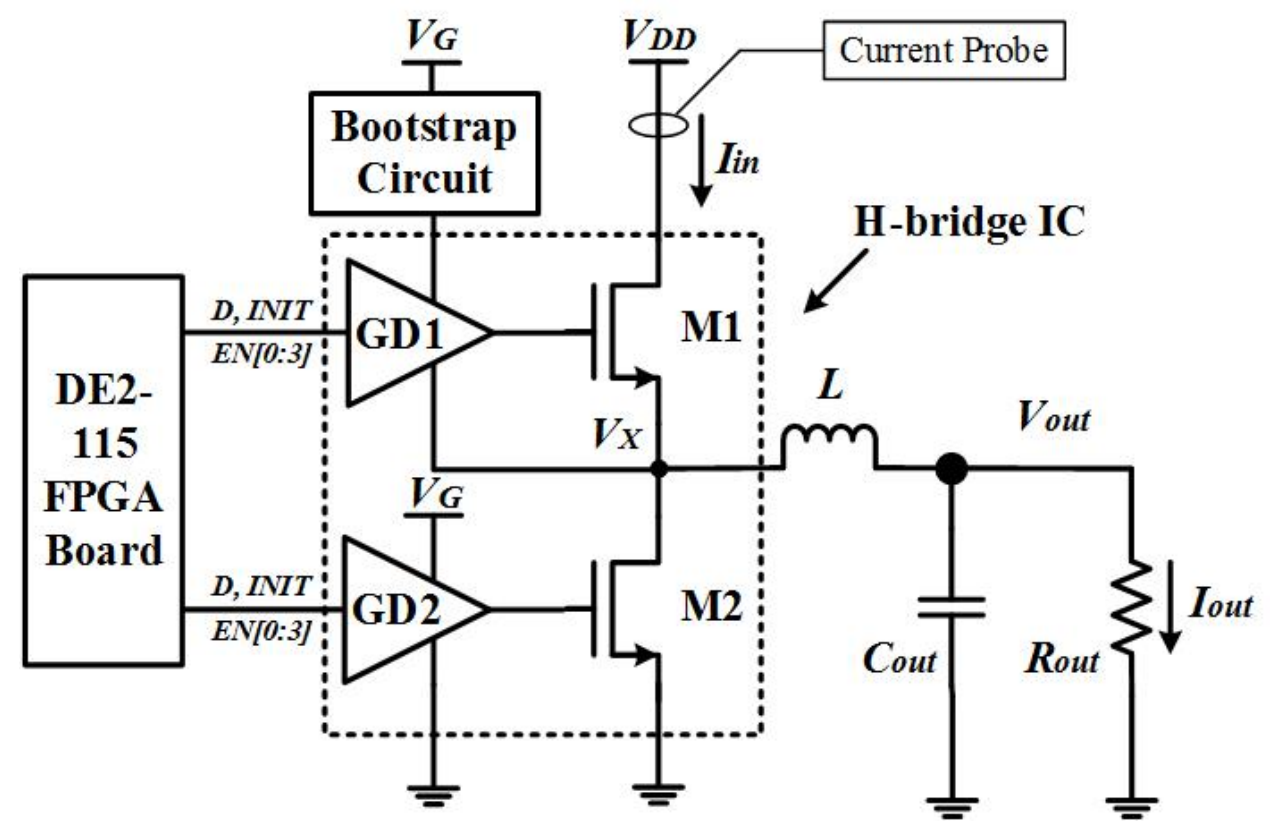

Figure 4.9: The buck converter circuit test bench.

\subsection{Experimental Setup}

In order to verify the performance of the segmented H-bridge, an experimental test bench is setup as shown in Fig. 4.9. For the work presented in this thesis, half of the H-bridge is examined at a time, with the other half disabled. Therefore, the experiments were performed with the H-bridge operating as a half bridge, with the circuit parameters as listed in Table 4.4. In order to measure the power conversion efficiency over a wide range of output current, an electronic load with programmable resistance is used. The input voltage, input current and output voltage are probed using an [Agilent MSO-X 3024A] oscilloscope. The rise-time and overshoot of the switching node voltage is monitored in order to verify the effectiveness of the segmented gate driver. A DE2-115 FPGA board with an Altera Cyclone $I V$ is used to generate the $1.8 \mathrm{~V}$ digital control signals. In order to investigate the effect of different gate driver output resistances on the CEMI produced by the system, the spectrum of the input current data are measured between $150 \mathrm{kHz}$ and $50 \mathrm{MHz}$. The PCB implementation details and whole testing setup can be found in Fig. 4.10 and 4.11, respectively. Two integrated H-bridge chips were used to build the output stage on the PCB since the high-side power transistors were not fully functioning after fabrication. 


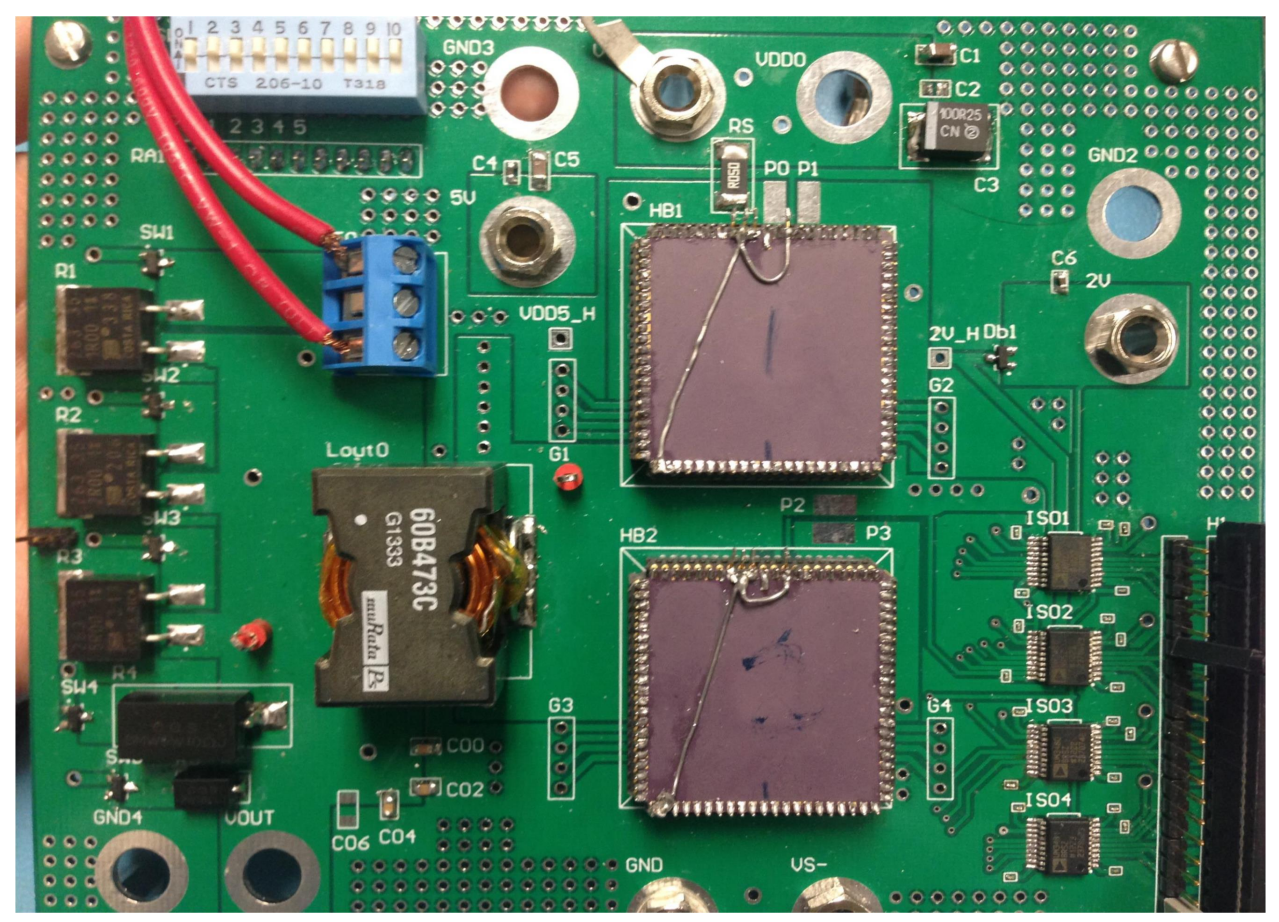

Figure 4.10: PCB implementation details.

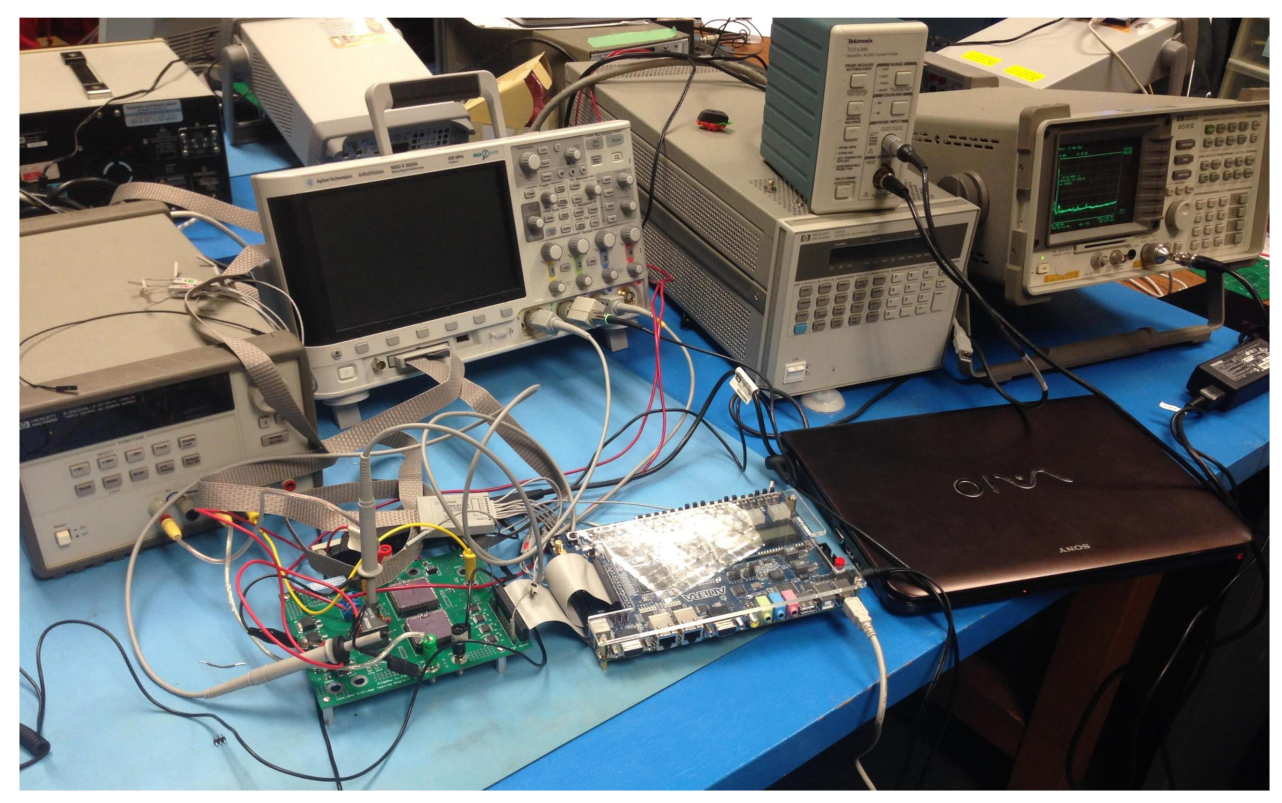

Figure 4.11: Overall experimental system setup. 
Table 4.4: Buck Converter Experimental Parameters.

\begin{tabular}{c|c|c}
\hline Parameter & Value & Units \\
\hline \hline Input Voltage $V_{D D}$ & 10 & $\mathrm{~V}$ \\
\hline Output Voltage $V_{\text {out }}$ & 2 & $\mathrm{~V}$ \\
\hline Load Current $I_{\text {out }}$ & $0.02-4$ & $\mathrm{~A}$ \\
\hline Switching Frequency $f_{s}$ & $100^{a}$ & $\mathrm{kHz}$ \\
\hline Output Inductor $L$ & 47 & $\mu \mathrm{H}$ \\
\hline Output Capacitance $C_{\text {out }}$ & 2 & $\mu \mathrm{C}$ \\
\hline Gate Voltage $V_{G}$ & 5 & $\mathrm{~V}$ \\
\hline Enabled Output Stage Segment(s) & 1 to 4 & - \\
\hline Driving Strategy EN[0:3] & {$[1111]$ to $[0001]$} & - \\
\hline
\end{tabular}

\footnotetext{
${ }^{a} \mathrm{~A}$ low switching frequency is chosen to accommodate the slow rise and fall times of the gate signal when a single gate drive segment is used.
}

\subsection{Switching Speed and Ringing}

Based on test bench as shown in Fig. 4.9, the behavior of the switching node voltage waveform is recorded during the turn-on of the high-side transistor for different gate driver output resistances. The switching node waveforms, as shown in Fig. 4.12, is monitored with a load current of $1 \mathrm{~A}$. It is evident that by decreasing the output resistance of the gate driver, the rise-time of the switching node drops from 472 ns to 33 ns. However, a lower gate driver output resistance also produces a greater amount of ringing at the switching node and increases the voltage overshoot from $0 \%$ to $33 \%^{1}$. Thuerefore, this inverse relationship leads to a trade-off between the turn-on time and the overshoot, as shown in Fig. 4.13.

In order to find an appropriate operating point, the output resistance $R_{\text {out }}$ is dynamically changed during the rising transition of the switching node. Two settings of $R_{\text {out }}$ are selected to demonstrate the benefits of the proposed driving strategy. $R_{\text {out }}$ is pre-set to a low value (all gate driver segments enabled with $\times 258$ driving strength) before the switching node starts to rise. The high-side power transistor is then turns on with the same fast speed provided by the $\times 258$ driving strength. Once the gate voltage transition is completed, a high $R_{\text {out }}$ is selected so that the ringing at the gate node can be damped. The switching node waveform for the proposed driving strategy can be found in Fig. 4.14, in which the overshoot and ringing is suppressed by $66 \%$ while the rise-time

\footnotetext{
${ }^{1}$ Overshoot voltage is defined as the ratio of the overshoot voltage value to the stable state step voltage.
} 


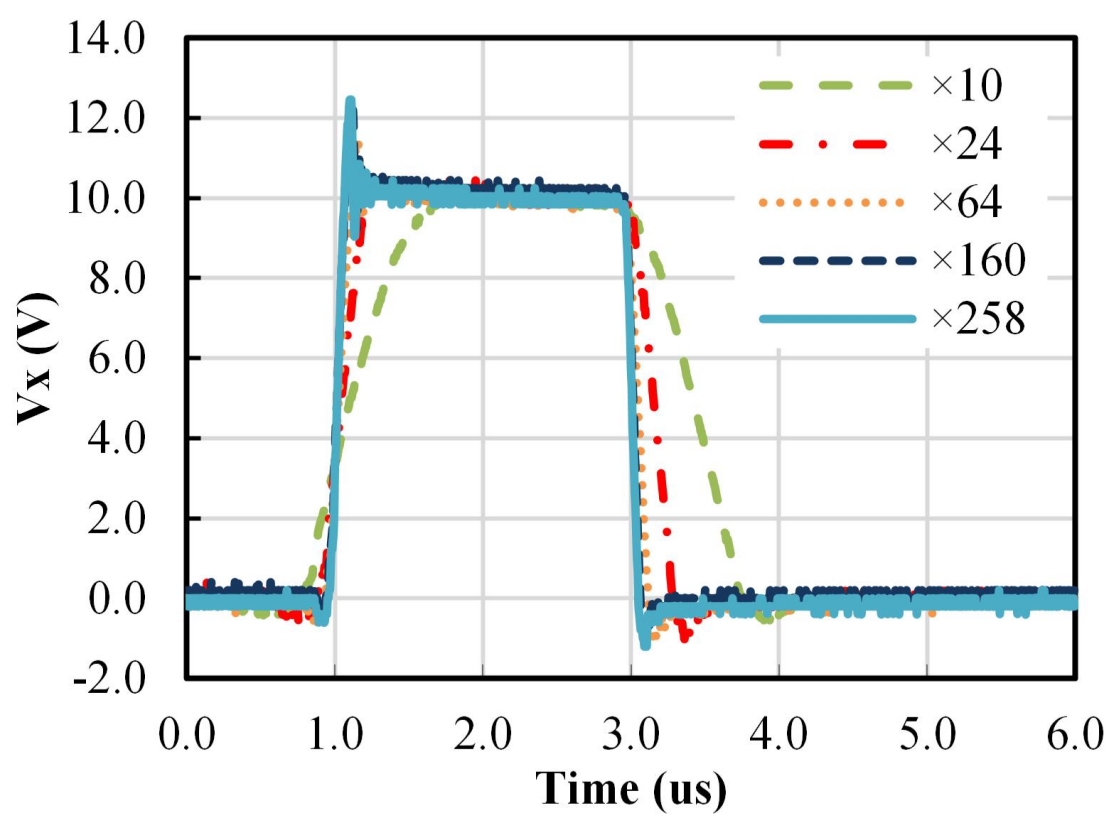

Figure 4.12: Switching node waveforms for different constant driving strength.

is maintained at $84 \mathrm{~ns}$.

\subsection{Power Conversion Efficiency Measurements}

The optimization strategy aims to find the best combination of gate driver configuration and output stage configuration to maximize the efficiency for a given current load. Two variables, namely $R_{\text {on }}$ of the output transistor and $R_{\text {out }}$ of the integrated gate driver, are investigated in this thesis. Experiments to record the power conversion efficiency versus load current are conducted in order to verify the simulation results in practical circuits.

Figure 4.15 plots the power conversion efficiency curves for three typical driving strengths when a half-bridge is operated with all output stage segments enabled. At light loads, small driving strength, which means high $R_{\text {out }}$, benefits from less power loss on gate driver circuit. At heavy load current, strong driving strength shows its advantage on switching loss reduction because of the short turn-on and turn-off transition time. A similar plots for the comparison of the efficiency with different output stage size is as shown in Fig. 4.16. A large power transistor is preferred for high load current, due to the small $R_{o n}$. At light loads, a small size output stage achieves higher power conversion efficiency since a small $C_{g s}$ reduces the gate driver loss according to (2.10).

Based on the discussions and experimental results mentioned above, the final H-bridge operation optimization is performed for load currents between $0.02 \mathrm{~A}$ and $4 \mathrm{~A}$. The power 


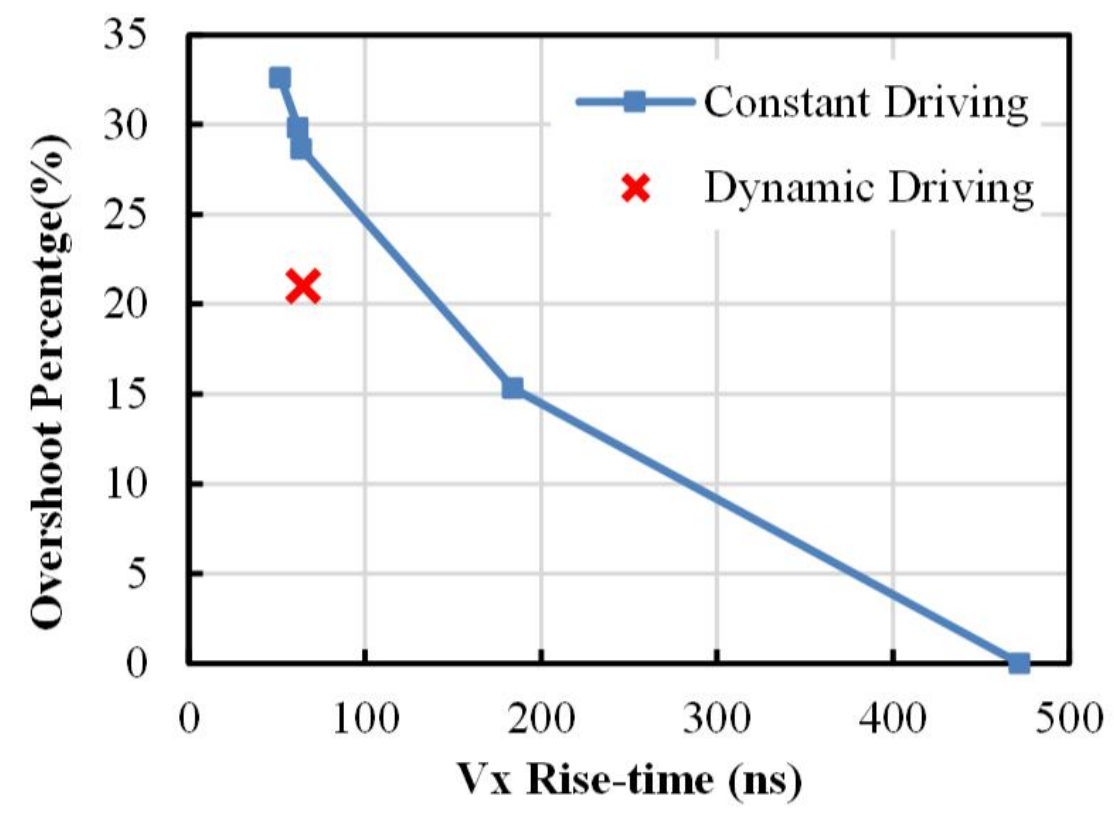

Figure 4.13: Rise time versus overshoot percentage.

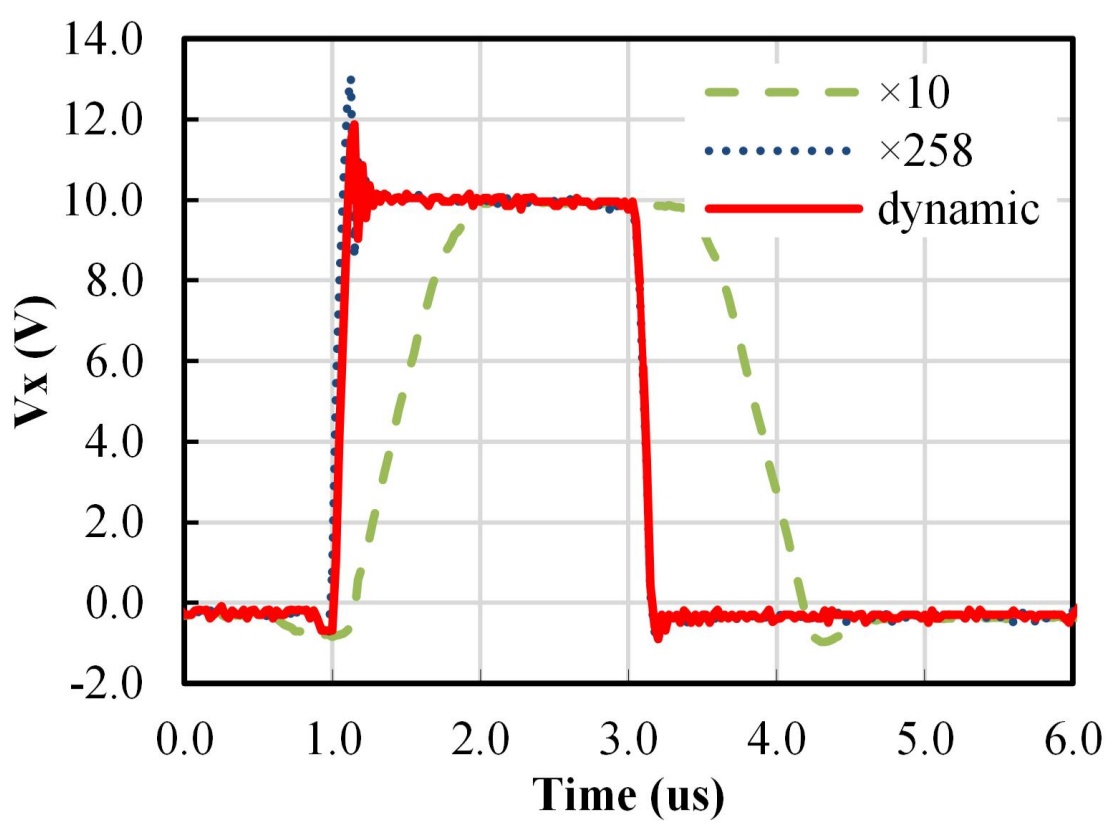

Figure 4.14: Switching node waveform for the proposed strategy. 


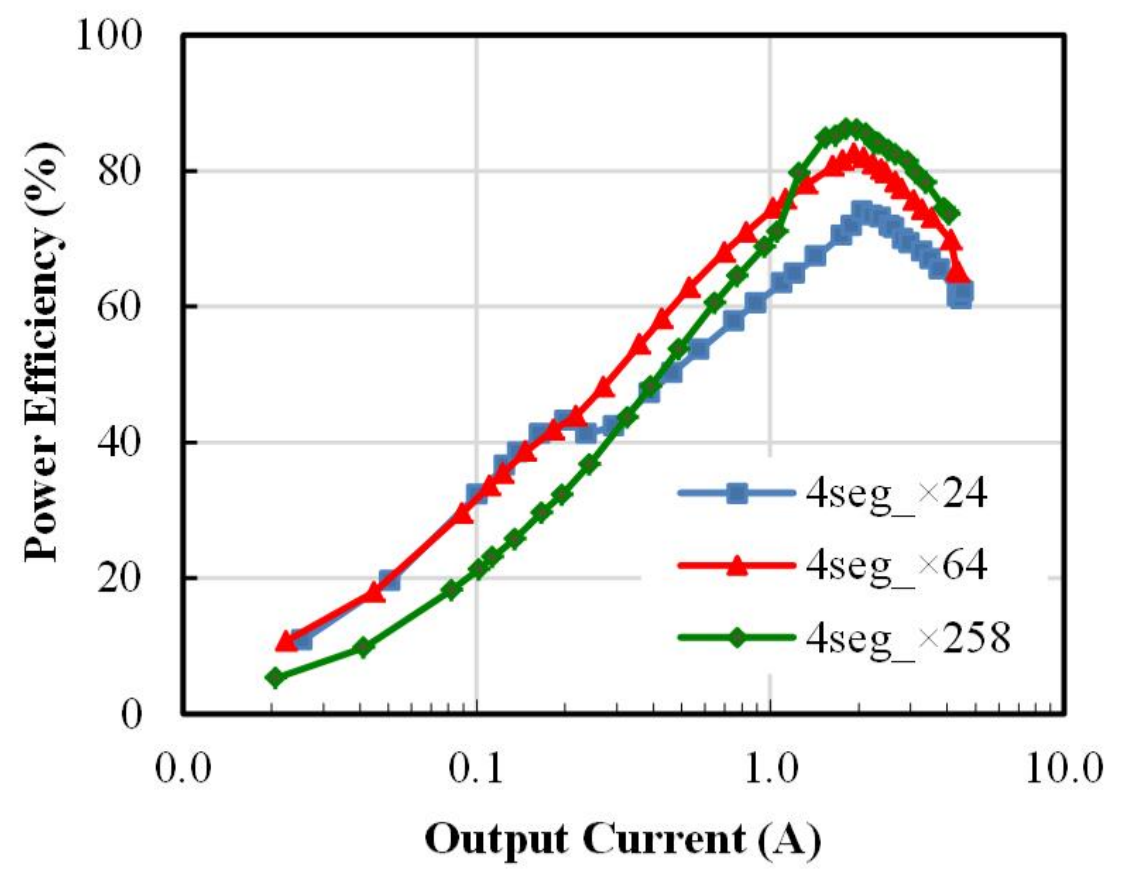

Figure 4.15: Power conversion efficiency versus load current for different gate driving strengths.

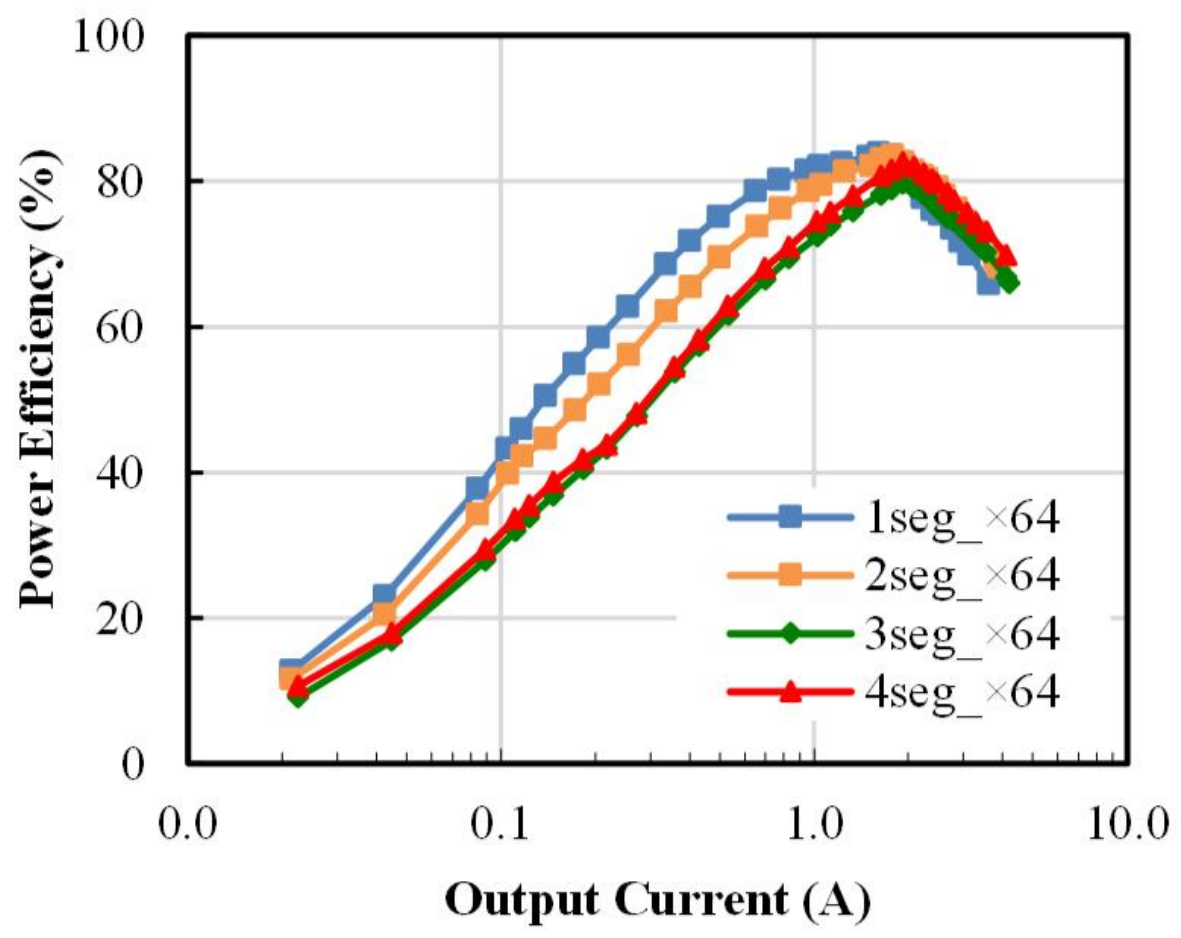

Figure 4.16: Power conversion efficiency versus load current for different output stage sizes. 


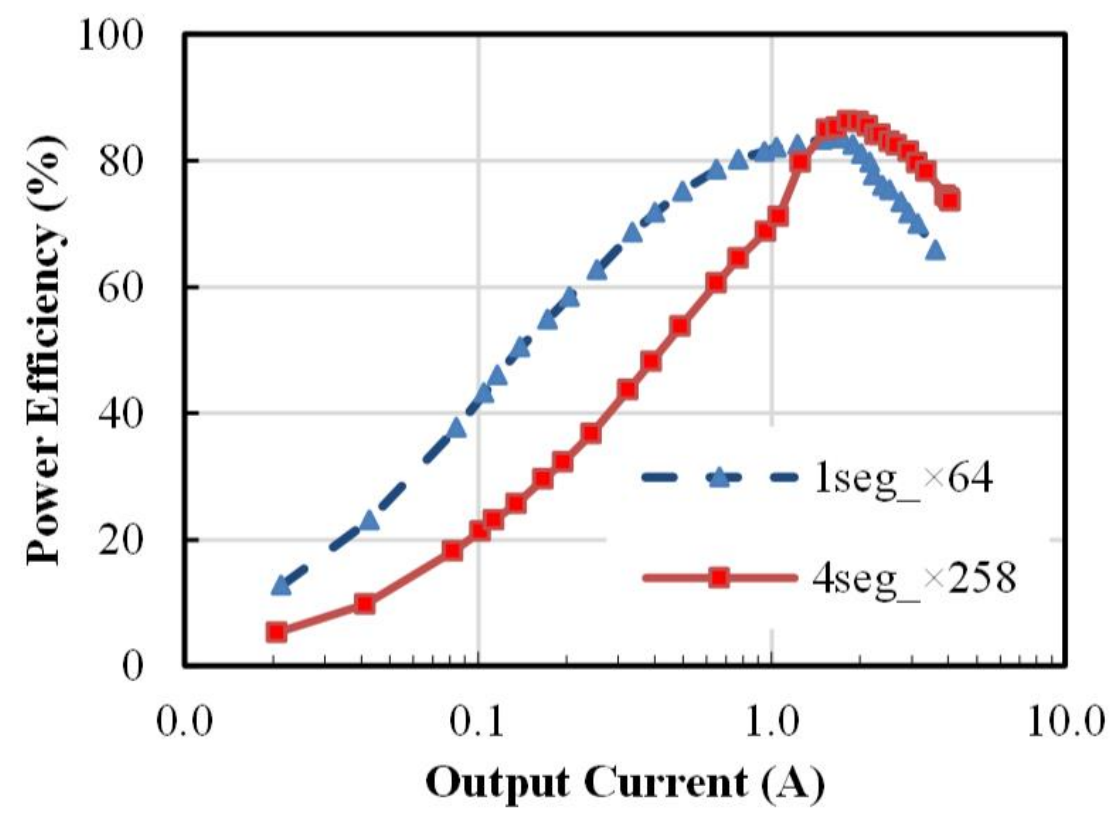

Figure 4.17: Efficiency versus load current for a half-bridge with the proposed operating strategy.

conversion efficiency for two typical combinations of output stage configuration and gate driver configuration is as plotted in Fig. 4.17. These two configurations are Configuration $A$ (when the output stage is configured such that only one segment is enabled and $\times 64$ driving strength is used) and Configuration $B$ (all output transistor segments and all gate driver segments are enabled).

For Configuration A, improvement in efficiency from $6 \%$ to $12 \%$ is observed when compared to Configuration B, for a load current of 0.02 A. Configuration A exhibits a $25 \%$ efficiency improvement at light load. However, for higher load currents, the advantages of Configuration $A$ is diminished due to higher conduction loss and switching loss. The highest recorded efficiency of $86 \%$ occurs when the load current is $2 \mathrm{~A}$ and is achieved by Configuration $B$, which shows an $8 \%$ efficiency improvement at $4 \mathrm{~A}$ when compared to Configuration A. The results in Fig. 4.17 demonstrate that for a given load current, a specific H-bridge configuration will maximize efficiency. Furthermore, as the design is digitally controlled, the combination of output stage and gate drive segments can be continuously optimized as the load current changes. 


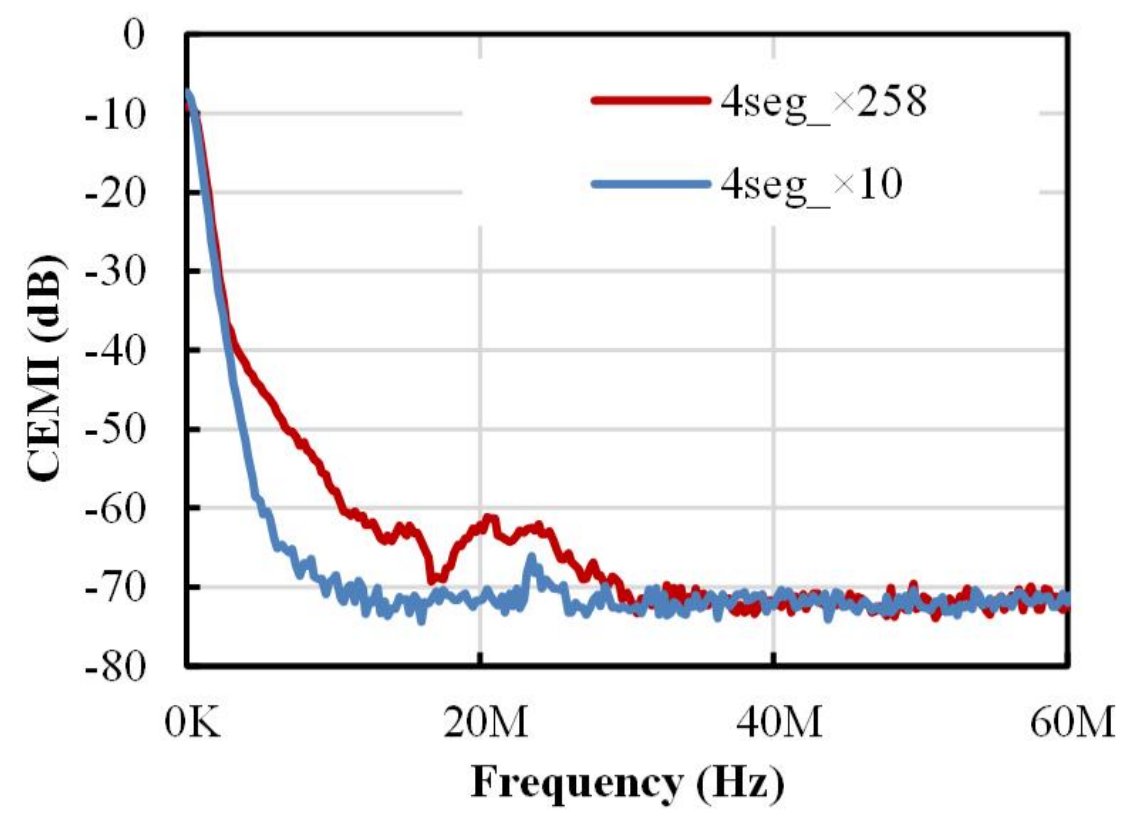

Figure 4.18: CEMI spectrum for two typical driving strength at 1 A load current.

\subsection{Switching Node Ringing on EMI Issue}

In order to investigate the conducted emissions of the strategy discussed in the previous section, the input current of the H-bridge was monitored using a [TektronixTCPA300] current probe. The CEMI was then determined by calculating the spectrum of the input current between $150 \mathrm{kHz}$ and $50 \mathrm{MHz}$. When the half-bridge, with all output stage segments enabled, is operated with the parameters as listed in Table 4.4, the CEMI spectrum for the highest driving strength $(\times 258)$ and lowest one $(\times 10)$ at 1 A load current is plotted in Fig. 4.18. The peak CEMI within a range of $20 \mathrm{MHz}$ to $40 \mathrm{MHz}$ is $5.2 \mathrm{dBm}$.

The proposed driving strategy for overshoot suppression is presented in Section 4.4. This section focuses on the CEMI mitigation at high switching frequency. Experimented half-bridge is operated at $1 \mathrm{MHz}$, with target load current of $1 \mathrm{~A}$. As described before, during the rising transition of the switching node, $R_{\text {out }}$ of the high-side gate driver is first set to a low value for fast switching and then switched to a high value to damp the ringing. By using the proposed gate driving strategy (dynamically switched from $\times 258$ to $\times 10$ ), an input current overshoot suppression of $6 \mathrm{~A}$ was observed, as shown in Fig. 4.19, when compared to the constant highest gate driving strength condition. The CEMI spectrum comparison can be found in Fig. 4.20. This figure clearly shows that within the frequency range of $20 \mathrm{MHz}$ to $40 \mathrm{MHz}$, the peak CEMI has a reduction of 5.5 


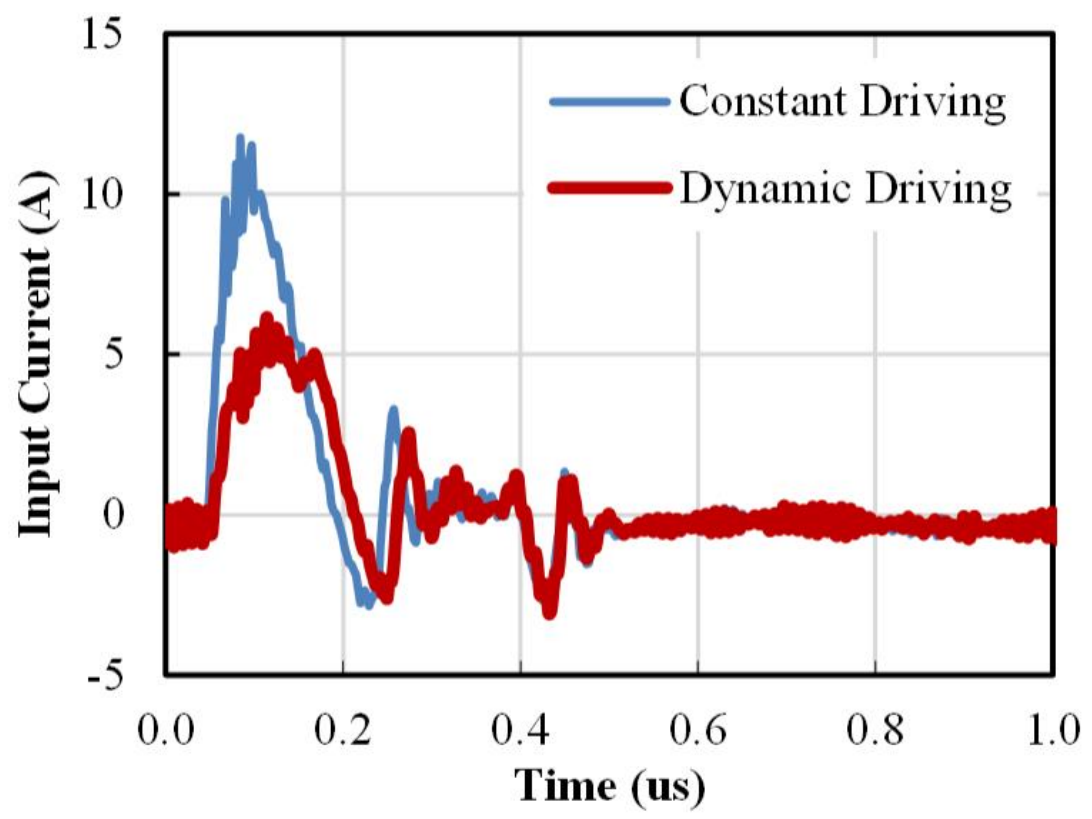

Figure 4.19: Input Current waveforms for different driving strategies.

$\mathrm{dBm}$. In addition, the power conversion efficiency for the dynamic driving strategy is $81 \%$. Compared to the $83 \%$ of the constant driving mode, the proposed strategy is not significantly degraded.

\subsection{Summary}

In this section, the segmented H-bridge IC with tunable gate driver is implemented and tested in a typical buck converter. The improvement in the power conversion efficiency is achieved by employing the proposed operation strategy. In addition, CEMI reduction is observed when dynamic driving strength is used. In Chapter 5, the conclusions of the research and future work are provided. 


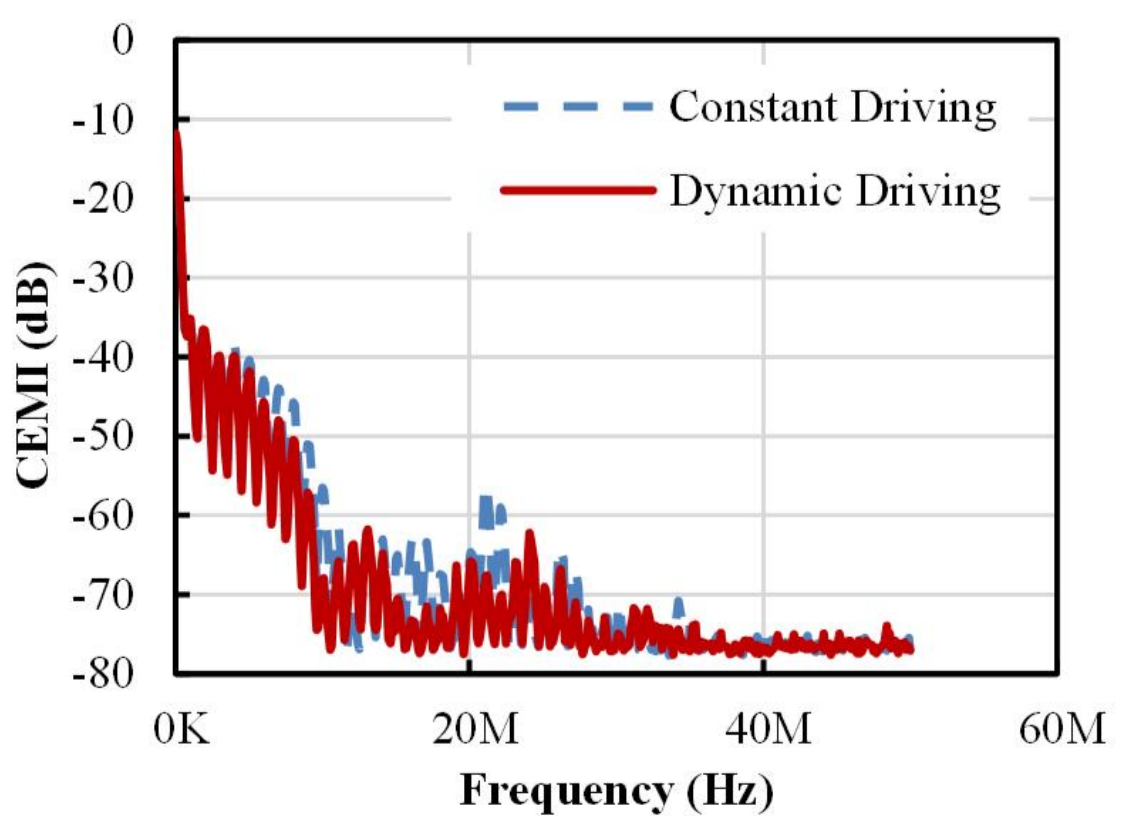

Figure 4.20: CEMI spectrum for different driving strategies. 


\section{Chapter 5}

\section{Conclusions}

The focus of this thesis is to optimize the output stage performance over a wide range of current, by operating with the best combination of segmented output stage and segmented gate drivers. EMI reduction by dynamically adjusting the output resistance of the gate driver is also investigated in this thesis.

\subsection{Conclusions}

This thesis investigates a segmented H-bridge design technique whereby the best combination of segmented output stage and gate driver are used to maximize the power conversion efficiency over a range of current loads. The design is capable of improving the power conversion efficiency by $25 \%$ under light load and $8 \%$ under heavy load conditions. Appropriate driving strength is also examined in this thesis for switching node ringing suppression. A reduction in CEMI by $5.5 \mathrm{dBm}$ without any significant power conversion efficiency degradation is obtained.

A paper titled "A segmented Output Stage H-Bridge IC with Tunable Gate Driver" was accepted at "The 26th Internatioanl Symposium On Power Semiconductor Devices and ICs".

\subsection{Future Work}

The segmented H-bridge design proposed in this thesis could be further studied to include the following topics.

- Broader load current range: The size for the unit segment of the output stage power transistor and the gate driver could be further optimized to suit for a broader 
range of load current. Decrease the unit segment size, but scale the total number of segments may help examine the efficiency at even lower or higher output power (i.e. less than $0.02 \mathrm{~A}$ or larger than $10 \mathrm{~A}$ ).

- Pulse frequency modulation (PFM): PFM can be utilized to help further improve the power conversion efficiency at light load conditions. The gate driver loss could be significantly reduced when H-bridge is operating in PFM mode.

- More states for driving strategy: The current design allows the switching between two states of the driving strength. Further work could be expanded into four or more states such that more than two settings of $R_{\text {out }}$ could be selected during a transition period. In addition, impact of different $T_{\text {pre }}$ and $T_{\text {post }}$ setting at the rising edge and the falling edge of the switching node can be examined.

- More practical applications: Only half-bridge is experimented so far. The testing can be expended to full H-bridge operation applied in more practical situations, e.g. motor drive, Class-D amplifiers, etc.. Benefits of the segmentation techniques can be further demonstrated in this way.

- Smart close loop system control on chip: The current design is experimented in a open loop circuit. If a close loop digital control could be implemented on chip, the best operating combination and driving strategy can be self-tuned, on-the-fly. In addition, this feature could greatly eliminates the propagation delay and noise brought by the long path from PCB to IC. 


\section{Bibliography}

[1] R. Erickson and D. Maksimovic, Fundamentals of power electronics. Springer, 2001.

[2] H-Bridges - the Basics. Internet: http://www.modularcircuits.com/blog/articles/hbridge-secrets/h-bridge-control/, Mar 2014.

[3] W. Ng, O. Trescases, and W. Guo, "Output stages for integrated dc-dc converters and power ics," in Electron Devices and Solid-State Circuits, 200\%. EDSSC $200 \%$. IEEE Conference on, pp. 91-94, Dec 2007.

[4] O. Trescases, W. Ng, H. Nishio, M. Edo, and K. T., "A digitally controlled dc-dc converter module with a segmented output stage for optimized efficiency," in Power Semiconductor Devices and IC's, 2006. ISPSD 2006. IEEE International Symposium on, pp. 409-413, 2006.

[5] F. Mihalic and D. Kos, "Reduced conductive emi in switched-mode dc ndash;dc power converters without emi filters: Pwm versus randomized pwm," Power Electronics, IEEE Transactions on, vol. 21, pp. 1783-1794, Nov 2006.

[6] K. Mainali and R. Oruganti, "Conducted emi mitigation techniques for switch-mode power converters: A survey," Power Electronics, IEEE Transactions on, vol. 25, no. 9, pp. 2344-2356, 2010.

[7] A. Shorten, A. Fomani, W. Ng, H. Nishio, and Y. Takahashi, "Reduction of conducted electromagnetic interference in smps using programmable gate driving strength," in Power Semiconductor Devices and ICs (ISPSD), 2011 IEEE 23rd International Symposium on, pp. 364-367, 2011.

[8] J. Wang, W. Ng, and O. Trescases, "Versatile capabilities of digitally controlled integrated dc-dc converters," in Circuits and Systems (ISCAS), 2011 IEEE International Symposium on, pp. 293-296, May 2011. 
[9] B. J. Baliga, "Power semiconductor device figure of merit for high-frequency applications," Electron Device Letters, IEEE, vol. 10, no. 10, pp. 455-457, 1989.

[10] W. Greig, Integrated circuit packaging, assembly and interconnections: trends and options. Springer, 2007.

[11] J. Rabaey, A. Chandrakasan, and B. Nikolic, Digital integrated circuits, vol. 2. Prentice hall Englewood Cliffs, 2002.

[12] PACKAGE INFORMATION. Internet: http://www.analog.com/en/quality-andreliability/content/package-information/fca.html, Mar 2014.

[13] T. Meade, D. O'Sullivan, R. Foley, C. Achimescu, M. Egan, and P. McCloskey, "Parasitic inductance effect on switching losses for a high frequency dc-dc converter," in Applied Power Electronics Conference and Exposition, 2008. APEC 2008. TwentyThird Annual IEEE, pp. 3-9, 2008.

[14] M. Pavier, A. Sawle, A. Woodworth, R. Monteiro, J. Chiu, and C. Blake, "High frequency dc:dc power conversion: the influence of package parasitics," in Applied Power Electronics Conference and Exposition, 2003. APEC '03. Eighteenth Annual IEEE, vol. 2, pp. 699-704 vol.2, 2003.

[15] G. Vasilescu, Electronic noise and interfering signals: principles and applications. Springer, 2005.

[16] E. Sicard, "Electromagnetic compatibility of integrated circuits," Microelectronics Journal, vol. 35, no. 6, pp. 485-486, 2004.

[17] P. Nicolae and M. Voinea, "Modeling and simulation of electromagnetic conducted emissions from buck converter with resistive load," in Applied and Theoretical Electricity (ICATE), 2012 International Conference on, 2012.

[18] D. Gonzalez, J. Balcells, A. Santolaria, J. Le Bunetel, J. Gago, D. Magnon, and S. Brehaut, "Conducted emi reduction in power converters by means of periodic switching frequency modulation," Power Electronics, IEEE Transactions on, vol. 22, no. 6, pp. 2271-2281, 2007.

[19] W. Ng, O. Trescases, and W. Guo, "Integrated power stages for switched mode power supplies," 
[20] J. Strydom, M. de Rooij, and J. Van Wyk, "A comparison of fundamental gate-driver topologies for high frequency applications," in Applied Power Electronics Conference and Exposition, 2004. APEC '04. Nineteenth Annual IEEE, vol. 2, pp. 1045-1052 vol.2, 2004.

[21] Z. Zhang, W. Eberle, Z. Yang, Y.-F. Liu, and P. Sen, "Optimal design of resonant gate driver for buck converter based on a new analytical loss model," Power Electronics, IEEE Transactions on, vol. 23, pp. 653-666, March 2008.

[22] A. Fomani, W. Ng, and A. Shorten, "An integrated segmented gate driver with adjustable driving capability," in Energy Conversion Congress and Exposition (ECCE), 2010 IEEE, pp. 2430-2433, 2010.

[23] O. Trescases, W. Guo, A. Prodic, and W. Ng, "Predictive efficiency optimization for dc-dc converters with highly dynamic digital loads," Power Electronics, IEEE Transactions on, vol. 23, no. 4, pp. 1859-1869, 2008.

[24] Y. Ismail, E. Friedman, and J. Neves, "Equivalent elmore delay for rlc trees," Computer-Aided Design of Integrated Circuits and Systems, IEEE Transactions on, vol. 19, pp. 83-97, Jan 2000.

[25] M. Ershov, A. Tcherniaev, Y. Feinberg, P. Lindorfer, W. French, and P. Hopper, "Numerical simulation of metal interconnects of power semiconductor devices," in Power Semiconductor Devices IC's (ISPSD), 2010 22nd International Symposium on, pp. 185-188, June 2010. 